\title{
PROBLEMS IN THE STEENROD ALGEBRA
}

\author{
R. M. W. WOOD
}

This article contains a collection of results and problems about the Steenrod algebra and related algebras acting on polynomials which non-specialists in topology may find of some interest. Although there are topological allusions throughout the article, the emphasis is on the algebraic development of the Steenrod algebra and its connections to the various topics indicated below.

\section{Contents}

1 Historical background 452

1.1 Early results on the Steenrod algebra 452

1.2 Algebras related to the Steenrod algebra 457

1.3 Remarks $\quad 458$

2 Differential operators and integral Steenrod squares $\quad 460$

2.1 The divided differential operator algebra 460

2.2 Global product formulae 466

2.3 The contragredient action of the differential operator algebra 468

2.4 Other sources of integral Steenrod squares 470

$\begin{array}{lll}2.5 & \text { Remarks } & 471\end{array}$

2.6 Problems 471

3 Symmetric functions and differential operators $\quad 471$

3.1 The algebraic Thom map 472

3.2 Products and conjugation in the symmetric algebra 473

$\begin{array}{lll}3.3 & \text { Remarks } & 475\end{array}$

3.4 Problems 476

4 Bases, excess and conjugates 476

4.1 The admissible basis $\quad 477$

4.2 The $P_{t}^{s}$ bases $\quad 479$

4.3 Arnon's C-basis 480

4.4 The Y-basis and Z-basis $\quad 480$

4.5 Wall's relations 481

4.6 Problems 483

5 The stripping technique $\quad 483$

5.1 Stripping in the Steenrod algebra 484

5.2 The strapping process 488

$\begin{array}{lll}5.3 \text { Problems } & 490\end{array}$

6 Iteration theory and nilpotence $\quad 490$

6.1 Iteration of power series $\quad 490$

6.2 Nilpotence in the Steenrod algebra 493

Received 10 October 1997; revised 25 March 1998.

1991 Mathematics Subject Classification 55S10. 
6.3 Annihilator ideals 494

6.4 Problems 495

7 The hit problem and invariant theory 495

7.1 The equivariant version for differential operators 496

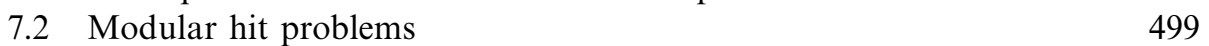

7.3 The hit problem for the Steenrod algebra 500

$\begin{array}{ll}\text { 7.4 Modular representations and splitting theory } & 502\end{array}$

$\begin{array}{ll}7.5 \text { Problems } & 504\end{array}$

8 The dual of $\mathscr{A}(n)$ and graph theory $\quad 504$

8.1 Graphical interpretation 505

$\begin{array}{ll}8.2 \text { Problems } & 507\end{array}$

9 The Steenrod group $\quad 507$

9.1 The group $U(1) \quad 507$

9.2 Remarks 508

9.3 Problems 508

10 Computing in the Steenrod algebra 508

$\begin{array}{ll}10.1 \text { Computing conjugates } & 509\end{array}$

$\begin{array}{ll}10.2 \text { Nilpotence questions } & 510\end{array}$

10.3 Problems 511

$\begin{array}{ll}\text { References } & 511\end{array}$

In Section 1 the scene is set with a few remarks on the early history of the Steenrod algebra $\mathscr{A}$ at the prime 2 from a topologist's point of view, which puts into context some of the problems posed later. In Section 2 the subject is recast in an algebraic framework, by citing recent work on integral versions of the Steenrod algebra defined in terms of differential operators. In Section 3 there is an explanation of how the divided differential operator algebra $\mathscr{D}$ relates to the classical theory of symmetric functions. In Section 4 some comments are made on a few of the recently discovered bases for the Steenrod algebra. The stripping technique in Section 5 refers to a standard action of a Hopf algebra on its dual, which is particularly useful in the case of the Steenrod algebra for deriving relations from relations when implemented on suitable bases. In Section 6 a parallel is drawn between certain elementary aspects of the iteration theory of quadratic polynomials and problems about the nilpotence height of families of elements in the Steenrod algebra. The hit problem in Section 7 refers to the general question in algebra of finding necessary and sufficient conditions for an element in a graded module over a graded ring to be decomposable into elements of lower grading. Equivariant versions of this problem with respect to general linear groups over finite fields have attracted attention in the case of the Steenrod algebra acting on polynomials. Similar problems arise with respect to the symmetric groups and the algebra $\mathscr{D}$. This subject relates to topics in classical invariant theory and modular representation theory. In Section 8 a number of statements about the dual Steenrod algebra are transcribed into the language of graph theory. In Section 9 a standard method is employed for passing from a nilpotent algebra over a finite field of characteristic $p$ to a $p$-group, and questions are raised about the locally finite 2-groups that arise in this way from the Steenrod algebra. Finally, in Section 10 there are a few comments on the use of a computer in evaluating expressions and testing relations in the Steenrod algebra.

The main thrust of research on the Steenrod algebra has naturally been con- 
cerned with modules over $\mathscr{A}$, since this lies at the heart of algebraic topology. The bibliographies of $[\mathbf{1 2 5}, \mathbf{1 4 5}, \mathbf{1 6 2}, \mathbf{1 8 9}]$ and $[170]$ give some indication of past work and current progress on the Steenrod algebra, and its applications to homotopy theory. The present article, on the other hand, has the very limited goal of addressing a few problems in a restricted area concerned with the internal structure of the Steenrod algebra and its action on polynomials. References are therefore rather selective. No attempt is made to broach the general theory of modules over the Steenrod algebra, higher-order operations, the homology of the Steenrod algebra, the Adams spectral sequence, or the theory of characteristic classes, except for occasional remarks. Some well-known material on Steenrod squares and Landweber-Novikov operations is rephrased in terms of differential operators, which form a recurrent theme throughout the article.

The standard reference for the Steenrod algebra is Steenrod and Epstein [189]. The books by Mosher and Tangora [145], Margolis [125] and, more recently, Schwartz [170] are specifically concerned with the Steenrod algebra and its applications to topology. The books by Switzer [195] and Gray [78] discuss the Steenrod algebra in the general context of homology and homotopy theory. The integral approach to Steenrod squares is closely related to operations in complex bordism theory, as Landweber noted in his original paper [111]. The Chicago Lecture Notes of Adams [5] describe Novikov's work on this subject, and a general survey of bordism theory can be found in Stong [190]. More recent references are Ravenel [162] and Kochman [101]. Ever since its inception, the Steenrod algebra has been allied to group cohomology theory and invariant theory [188]. The book on invariant theory by Larry Smith [183] contains informative chapters on the Steenrod algebra, and likewise the books of Adem and Milgram [12] and Benson [23] on the cohomology of groups. A standard reference for symmetric functions is Macdonald's book [121], and the representation theory of symmetric groups and general linear groups over finite fields is treated in the book of James and Kerber [90]. The differential operator approach to the Steenrod algebra and the Landweber-Novikov algebra touches on certain combinatorial material which can be found in standard texts such as Aigner [13], Cameron [37], Comtet [47], Goulden and Jackson [77] and Henrici [80], as well as the classic text of MacMahon [122]. Recent work on the Steenrod algebra and the Landweber-Novikov algebra in relation to combinatorics can be found in $[163,164,165,169]$.

Steenrod's original methods of defining the Steenrod algebra [187] at various primes, and the contributions of the early pioneers to the development of the Steenrod algebra, are expounded in Dieudonnés history of algebraic and differential topology [59]. The Steenrod algebra is a graded Hopf algebra, for which the papers of Milnor [131] and Milnor and Moore [133] are standard references. The differential operator approach to the Steenrod algebra impinges on areas of mathematics concerning $\mathscr{D}$-modules, Lie algebras, deformation of Hopf algebras, quantum groups and the Weyl algebra. Relevant information can be found in a number of sources: $[1,27,48,76,97,124,125,127,129,144,194]$.

The present article expands on material found in [221], but stems originally from a talk entitled 'Facts and fancies in the Steenrod algebra' delivered during the topology conference at Göttingen in 1991. I am grateful to Larry Smith for his hospitality and useful comments on the subject of Steenrod squares. My thanks are also due to colleagues in Manchester: Mike Prest, Bob Sandling and Grant Walker for much help on representation theory, and Nige Ray for guidance on combinatorial 
matters, especially on the relationship between the differential operator algebra and the Landweber-Novikov algebra. Part of the present work has been supported by EPSRC grant GR/K 05856.

\section{Historical background}

In 1947 Steenrod [186] introduced certain linear operators of ordinary cohomology theory $\mathbf{H}^{*}$ over $\mathbf{F}_{2}$ defined in terms of cocycles in a simplicial cochain complex by modifying the Alexander-Čech-Whitney formula for the cup-product construction. Subsequently called Steenrod squares,

$$
S q^{k}: \mathbf{H}^{n}(X) \rightarrow \mathbf{H}^{n+k}(X),
$$

these operators are natural transformations defined for $k, n \geqslant 0$ on the cohomology of a topological space $X$, and they commute with suspension. Such operations are called stable.

1.1 Early results on the Steenrod algebra. Cartan discovered a formula for evaluating a Steenrod square on the cup product of cohomology classes $f, g$.

Theorem 1.1 (Cartan [43]). $S q^{n}(f g)=\sum_{0 \leqslant r \leqslant n} S q^{r}(f) S q^{n-r}(g)$.

Using earlier work of P. A. Smith and M. Richardson [167] on the homology of cyclic products of topological spaces, Thom [198] and $\mathrm{Wu}[\mathbf{2 2 4}, 222]$ gave a characterisation of the Steenrod squares in terms of the Cartan formula, the cohomology boundary operator, and the normalisation conditions, usually called 'unstability' conditions, which state that $S q^{k}$ is the squaring function on $\mathbf{H}^{k}$, and zero on $\mathbf{H}^{n}$ for $k>n$. Wu introduced Smith-Richardson homology operations $S m_{k}: \mathbf{H}_{n}(X) \rightarrow \mathbf{H}_{n-k}(X)$ which were later $[\mathbf{2 2 5}, \mathbf{2 2 6}]$ seen to be related, via Kronecker duality, to $\chi\left(S q^{k}\right)$, where $\chi$ is a certain conjugation operation in the Steenrod algebra introduced by Thom in his study of manifolds [200].

Serre [171] showed that the Steenrod squares generate all stable operations in the cohomology theory. From a topological point of view, $\mathscr{A}$ is the algebra of stable operations of $\mathbf{H}^{*}$ over $\mathbf{F}_{2}$ generated by the $S q^{n}$ under composition, subject to relations which vanish on the cohomology of all spaces $X$.

$\mathrm{Wu}[\mathbf{2 2 4}, \mathbf{2 2 2}]$ conjectured certain relations among the squaring operations which were proved by Adem.

Theorem 1.2 (Adem $[\mathbf{1 0}, \mathbf{1 1}]$ ). All relations in the Steenrod algebra are generated by the set of Adem relations

$$
S q^{i} S q^{j}=\sum_{0 \leqslant k \leqslant[i / 2]}\left(\begin{array}{c}
j-k-1 \\
i-2 k
\end{array}\right) S q^{i+j-k} S q^{k}
$$

for $0<i<2 j$, where $[i / 2]$ denotes the greatest integer $\leqslant i / 2$, and the binomial coefficients are taken modulo 2.

The Steenrod algebra $\mathscr{A}$ can be formally defined as the graded associative algebra generated over $\mathbf{F}_{2}$ by symbols $S q^{k}$, for $k \geqslant 0$, subject to the Adem relations and $S q^{0}=1$. The grading of the monomial $S q^{i_{1}} S q^{i_{2}} \cdots S q^{i_{k}}$ is $i_{1}+\cdots+i_{k}$. The formal 
length of the monomial is $k$ if all $i_{j}$ are positive. The Adem relations preserve the grading and never increase length of monomials when the re-write rules are applied to $S q^{i} S q^{j}$ for $0<i<2 j$.

In his work on the cohomology of Eilenberg-MacLane spaces for the group of order 2, Serre $[\mathbf{1 7 1}, 44]$ gave a method of deriving the Adem relations in terms of a faithful representation of $\mathscr{A}$ on the cohomology of the infinite product of infinite real projective spaces. Let $\mathbf{W}=\mathbf{Z}\left[x_{1}, \ldots, x_{n}, \ldots\right]$ denote the polynomial algebra over the integers in a countable number of commuting variables $x_{n}$ graded by degree. Let $\mathbf{W}(n)$ be the subalgebra of $\mathbf{W}$ generated by the first $n$ variables, and let $\mathbf{W}_{d}$ be the abelian subgroup of homogeneous polynomials of degree $d$, with corresponding notation for the polynomial algebra $\mathbf{W} \otimes R=R\left[x_{1}, \ldots, x_{n}, \ldots\right]$ over a commutative ring $R$. The cohomology ring of the infinite product of infinite real projective spaces is isomorphic to $\mathbf{W} \otimes \mathbf{F}_{2}$. This furnishes the link with the purely algebraic development of the Steenrod algebra as expressed by the following characterisation of the Steenrod squares by their action on polynomials.

Lemma 1.3. The squaring operations are uniquely determined by the following conditions.

(1) $S q^{k}: \mathbf{W}_{d} \otimes \mathbf{F}_{2} \rightarrow \mathbf{W}_{d+k} \otimes \mathbf{F}_{2}$ are linear transformations.

(2) $S q^{0}$ is the identity.

(3) $S q^{1} x_{i}=x_{i}^{2}$ and $S q^{k} x_{i}=0$ for $k>1, i>0$.

(4) $S q^{n}(f g)=\sum_{0 \leqslant r \leqslant n} S q^{r}(f) S q^{n-r}(g)$ for polynomials $f$ and $g$.

The Steenrod squares preserve $\mathbf{W}(n) \otimes \mathbf{F}_{2}$ for each $n$. In principle, the Cartan formulae enable the evaluation of a squaring operation on any polynomial, by induction on degree. For example, if $f$ is a homogeneous polynomial of degree $k$, then $S q^{k}(f)=f^{2}$, which explains the terminology squaring operation. Furthermore, $S q^{k}(f)=0$ if $k>\operatorname{deg}(f)$.

The Steenrod algebra may therefore be viewed as the algebra of linear operators on polynomials generated under composition by the Steenrod squares, subject to the relations which vanish on all polynomials.

Serre introduced the notions of admissible and excess. The monomial

$$
S q^{i_{1}} \cdots S q^{i_{k}}
$$

is called admissible if $i_{j-1} \geqslant 2 i_{j}$ for $2 \leqslant j \leqslant k$ and $i_{k} \geqslant 1$. The Adem relations lead to an additive basis for $\mathscr{A}$.

THeOREM 1.4 (Serre [171]). The set of admissible monomials and $S q^{0}$ is an additive basis of the Steenrod algebra.

In grading 0 , the Steenrod algebra is the ground field $\mathbf{F}_{2}$, and $\mathscr{A}$ is finite dimensional in each grading. (In topologists' language, the Steenrod algebra is connected and of finite type.) For example, a vector space basis up to grading 3 is given by

$$
S q^{0}, \quad S q^{1}, \quad S q^{2}, \quad S q^{3}, \quad S q^{2} S q^{1} .
$$

Serre found a test for a linear combination of monomials of squaring operations to be the zero element in the Steenrod algebra $[\mathbf{1 4 5}, \mathbf{1 8 9}]$. 
THEOREM 1.5 ([189, Chapter 1]). A combination of squaring operations of grading $\leqslant n$ is identically zero if and only if it vanishes on the product of variables $x_{1} x_{2} \cdots x_{n}$.

The excess of an element $E$ in $\mathscr{A}$ is defined as the smallest positive integer $s$ such that $E\left(x_{1} x_{2} \cdots x_{s}\right) \neq 0$. The excess of the admissible element $S q^{i_{1}} \cdots S q^{i_{k}}$ is $2 i_{1}-d$, where $d=i_{1}+\cdots+i_{k}$ is the grading of the element.

The Adem relations also lead to a minimal algebraic generating set for $\mathscr{A}$. An element in grading $g$ is described as indecomposable if it cannot be written as a linear combination of products of elements of grading lower than $g$.

Theorem 1.6 ([189, Chapter 1]). The squaring operations $S q^{2^{k}}$ are indecomposable, and $\mathscr{A}$ is generated as an algebra by $S q^{0}$ and the $S q^{2^{k}}$ for $k \geqslant 0$.

In the decade 1950-60, the Steenrod algebra became one of the most powerful tools in algebraic topology. For example, Adams solved the Hopf invariant one problem [3], thereby proving that non-singular bilinear maps $\mathbf{R}^{n} \times \mathbf{R}^{n} \rightarrow \mathbf{R}^{n}$ exist only for dimensions $n=1,2,4,8$, where they are realised by real, complex, quaternionic and Cayley multiplication. The fact that such maps can exist only in dimensions of the form $2^{k}$ is already a consequence of Theorem 1.6. Adams went deeper into the structure of the Steenrod algebra, by decomposing $S q^{2^{k}}$ for $k>3$ in terms of certain 'secondary' operations to finally settle the problem. He also developed his spectral sequence $[\mathbf{2}, \mathbf{1 4 5}]$, which relates the homology of the Steenrod algebra to the 2-primary part of the stable homotopy groups of spheres. In another direction, Thom [200, 197] linked bordism theory of manifolds and characteristic classes of vector bundles to the Steenrod algebra [134].

The internal structure of $\mathscr{A}$ was investigated by Adams in Section 5 of [2]. $\mathrm{He}$ proved, in particular, that any finite collection of elements in $\mathscr{A}$ generates a finite subalgebra. In fact, $\mathscr{A}$ is a local algebra with elements of positive grading as its maximal ideal, and this is a nil-ring. For each non-zero element $E$ in $\mathscr{A}$ of positive grading, there is a positive integer $h$, the height of $E$, such that $E^{h}=0$ and $E^{h-1} \neq 0$.

Milnor [131] established that $\mathscr{A}$ has a natural coproduct which makes it into a Hopf algebra and incorporates Thom's involution as the conjugation. The coproduct $\psi$ and conjugation $\chi$ in $\mathscr{A}$ are determined on Steenrod squares by

$$
\psi\left(S q^{k}\right)=\sum_{0 \leqslant i \leqslant k} S q^{i} \otimes S q^{k-i}, \quad \chi\left(S q^{k}\right)=\sum_{1 \leqslant i \leqslant k} S q^{i} \chi\left(S q^{k-i}\right)
$$

and $\chi\left(S q^{0}\right)=1$. Conjugation is an anti-automorphism of the Steenrod algebra, and its square is the identity.

Milnor defined the element $\xi_{n}$ in the dual, $\mathscr{A}^{*}$, of $\mathscr{A}$ as the dual of

$$
S q^{2^{n-1}} S q^{2^{n-2}} \cdots S q^{2} S q^{1}
$$

with respect to the admissible basis. The grading of $\xi_{n}$ is $2^{n}-1$. He established that the dual of the coalgebra $\mathscr{A}$ is the polynomial algebra $\mathbf{F}_{2}\left[\xi_{1}, \xi_{2}, \ldots\right]$, and this gives rise to Milnor's basis of $\mathscr{A}$ by dualising the monomial basis of $\mathscr{A}^{*}$. A vector $R=\left(r_{1}, r_{2}, \ldots, r_{k}\right)$ of non-negative integers is identified with the sequence $\left(r_{1}, r_{2}, \ldots, r_{k}, \ldots\right)$ with trailing zeros after $r_{k}$. Only sequences with a finite number of 
non-zero terms are considered. The notation

$$
S q(R)=S q\left(r_{1}, r_{2}, \ldots, r_{k}\right)
$$

is used to denote the dual of the monomial $\xi_{1}^{r_{1}} \xi_{2}^{r_{2}} \cdots \xi_{k}^{r_{k}}$. If $r_{k} \neq 0$, then $k$ is the length of $S q(R)$. The grading of this element is

$$
r_{1}+3 r_{2}+7 r_{3}+\cdots+\left(2^{k}-1\right) r_{k} .
$$

In particular, $S q(r)=S q^{r}$. For example, the Milnor basis up to grading 3 consists of the elements

$$
S q(0), \quad S q(1), \quad S q(2), \quad S q(3), \quad S q(0,1)=S q^{3}+S q^{2} S q^{1} .
$$

The dimensions of the homogeneous parts of the dual Steenrod algebra, and therefore of the Steenrod algebra itself, are given by the coefficients of the Poincare series $\prod_{n=1}^{\infty}\left(1-t^{2^{n}-1}\right)^{-1}$.

Milnor constructed finite Hopf subalgebras $\mathscr{A}(n)$ of $\mathscr{A}$ by dualising certain quotients of the polynomial algebra $\mathscr{A}^{*}$. It turns out that $\mathscr{A}(n)$ is generated by the squares $S q^{2^{j}}$ for $0 \leqslant j \leqslant n$ and $S q^{0}$. The Steenrod algebra is the union of the finite Hopf algebras $\mathscr{A}(n)$. The Milnor basis is compatible with the subalgebras $\mathscr{A}(n)$, in the sense that $\mathscr{A}(n)$ is generated additively by the Milnor basis elements that lie in it. Since every element of the Steenrod algebra belongs to $\mathscr{A}(n)$ for some $n$, and the $\mathscr{A}(n)$ are finite dimensional vector spaces, the nilpotence result for the Steenrod algebra follows immediately. Other elementary facts that come from the Adem relations, or by dualising, are that $\mathscr{A}$ has a trivial centre and its abelianisation is a divided polynomial algebra on one generator [189].

The notation $P_{t}^{s}$ has become standard for the element $S q\left(\ldots, 2^{s}, \ldots\right)$, where 2 occupies position $t$, and the other positions are 0. Occasionally, $S q_{t}(R)$ is used to denote the Milnor basis element corresponding to the sequence with $r_{i}$ in position it and zeros elsewhere. For example,

$$
P_{t}^{s}=S q_{t}\left(2^{s}\right), \quad P_{t}^{0}=S q_{t}(1)=S q(0, \ldots, 0,1,0, \ldots) .
$$

It is convenient to write $\xi_{0}=1$. The coproduct and conjugation formulae in the dual algebra $\mathscr{A}^{*}$ are determined on generators by

$$
\psi\left(\xi_{k}\right)=\sum_{0 \leqslant i \leqslant k} \xi_{k-i}^{2^{i}} \otimes \xi_{i}, \quad \chi\left(\xi_{k}\right)=\sum_{0 \leqslant i \leqslant k-1} \xi_{k-i}^{2^{i}} \chi\left(\xi_{i}\right)
$$

and $\chi\left(\xi_{0}\right)=1$. The primitives in $\mathscr{A}^{*}$ are the elements $\xi_{1}^{2^{k}}$, and the $\xi_{i}$ are indecomposable. The primitives in the Steenrod algebra are the $P_{k}^{0}$, and the $S q^{2^{k}}$ are indecomposable.

Milnor also produced an explicit product formula for basis elements

$$
S q\left(r_{1}, \ldots, r_{m}\right), \quad S q\left(s_{1}, \ldots, s_{n}\right) .
$$

Consider matrices

$$
X=\left(\begin{array}{cccc}
* & x_{01} & x_{02} & \cdots \\
x_{10} & x_{11} & x_{12} & \cdots \\
x_{20} & x_{21} & x_{22} & \cdots \\
\vdots & \vdots & \vdots & \ddots
\end{array}\right),
$$

where the $x_{i j}$ are non-negative integers, with $x_{00}$ omitted. The $x_{i j}$ are subject to the 
constraints

$$
r_{i}=\sum_{v \geqslant 0} 2^{v} x_{i v}, \quad s_{j}=\sum_{u \geqslant 0} x_{u j},
$$

where $i, j \geqslant 1$. Let $t_{l}=\sum_{0 \leqslant i \leqslant l} x_{i, l-i}$ denote the diagonal sums of the matrix $X$, and let

$$
S q[X]=S q\left(t_{1}, \ldots, t_{l}, \ldots\right) .
$$

Multinomial coefficients are defined by

$$
b_{l}(X)=\frac{t_{l} !}{x_{0 l} ! x_{1, l-1} ! \cdots x_{l 0} !},
$$

and the notation is extended to $b(X)=b_{1}(X) b_{2}(X) \cdots$. Then Milnor's formula reads as follows.

THEOREM 1.7 (Milnor [131]).

$$
S q\left(r_{1}, \ldots, r_{m}\right) S q\left(s_{1}, \ldots, s_{n}\right)=\sum_{X} b(X) S q[X],
$$

where the summation is taken over all matrices $X$ subject to the given constraints, and the multinomial coefficients are reduced modulo 2.

Although the above descriptions of relations, products, coproducts and conjugation in the Steenrod algebra and its dual are very explicit, in practice several problems about the structure of $\mathscr{A}$ and its action on polynomials have continued to occupy the interest of topologists. Let $\mathscr{A}^{+}$denote the elements of positive grading in $\mathscr{A}$.

Problems 1.8 .

(1) Find the heights of specified elements in $\mathscr{A}$.

(2) Find defining relations for $\mathscr{A}$ in terms of the $S q^{2^{n}}$.

(3) Find efficient methods of evaluating excess and conjugation.

(4) Investigate transition matrices between various bases of $\mathscr{A}$.

(5) Identify the image of the action of $\mathscr{A}^{+}$on $\mathbf{W} \otimes \mathbf{F}_{2}$.

(6) Investigate integral lifts of the Steenrod squares and algebras related to the Steenrod algebra.

In 1960, Wall found a minimal defining set of relations for $\mathscr{A}$ involving just the $S q^{2^{n}}$.

THEOREM 1.9 (Wall [213]).

$$
S q^{2^{i}} S q^{2^{i}} \equiv S q^{2^{i-1}} S q^{2^{i}} S q^{2^{i-1}}+S q^{2^{i-1}} S q^{2^{i-1}} S q^{2^{i}}, \quad S q^{2^{i}} S q^{2^{j}} \equiv S q^{2^{j}} S q^{2^{i}}
$$

for $0 \leqslant j \leqslant i-2$, where the equivalences are modulo $\mathscr{A}(i-1)$.

Wall showed how these relations lead to a basis of $\mathscr{A}$ involving monomials in the $S q^{2^{n}}$, but no closed formulae were proposed for the relations themselves. Since then, several new bases of $\mathscr{A}$ have emerged $[\mathbf{1 7}, 143,220]$, and closed forms have been discovered for Wall's relations. To describe Wall's basis, let

$$
Q_{k}^{n}=S q^{2^{k}} S q^{2^{k+1}} \cdots S q^{2^{n}}
$$

for $n \geqslant k$. 
THEOREM 1.10 (Wall [213]). The set of monomials $Q_{k_{0}}^{n_{0}} Q_{k_{1}}^{n_{1}} \cdots Q_{k_{a}}^{n_{a}}$, where the vectors $\left(n_{i}, k_{i}\right)$ are in left lexicographic order

$$
\left(n_{a}, k_{a}\right)<\left(n_{a-1}, k_{a-1}\right)<\ldots<\left(n_{0}, k_{0}\right),
$$

is a basis of the Steenrod algebra.

Wall applied his results to the evaluation of the first few cohomology groups of the Steenrod algebra [213], and to settle some questions raised by Toda [201] about right multiplication in $\mathscr{A}$ by $S q^{2^{n}}$.

Cohen [46] showed how to obtain the Adem relations from the Milnor product formula. Of special interest are the following particular cases.

EXAMPLE 1.11. $S q^{2 m-1} S q^{m}=0$ for $m>0$

Kristensen $[106,104,105]$ introduced a process which he called differentiation, but which we shall call stripping, for deriving relations from relations in the Steenrod algebra. The stripping process is an action of the dual of the Steenrod algebra on $\mathscr{A}$ defined by

$$
\mathscr{A}^{*} \otimes \mathscr{A} \stackrel{1 \otimes w}{\longrightarrow} \mathscr{A}^{*} \otimes \mathscr{A} \otimes \mathscr{A} \stackrel{\kappa \otimes 1}{\longrightarrow} \mathscr{A},
$$

where $\psi$ is the coproduct in $\mathscr{A}$, and $\kappa$ is Kronecker evaluation of a vector space on its dual [78]. The element $\xi_{1}$ in the dual Steenrod algebra acts as a derivation. For example, the effect of stripping the relation $S q^{2 m-1} S q^{m}=0$ by $\xi_{1}$ is to produce the relation $S q^{2 m-2} S q^{m}+S q^{2 m-1} S q^{m-1}=0$. We shall explain later how this process works more generally. At this point, we state Kristensen's result.

Theorem 1.12 (Kristensen [106]). The Adem relations in the Steenrod algebra are obtained from the basic relations $S q^{2 m-1} S q^{m}=0$ by iterated stripping with $\xi_{1}$.

In fact, in any of the finite algebras $\mathscr{A}(n)$, the relations are generated by stripping just one relation $S q^{2^{m+1}-1} S q^{2^{m}}=0$ if $m$ is large enough, but then stripping by $\xi_{2}$ is also needed. The Adem relations are obtained by induction on degree and the stripping process.

The years 1960-70 saw the development of generalised cohomology theories, $K$ theory, bordism theory, $B P$-theory, stable homotopy theory and the general theory of spectra. New cohomology theories have continued to emerge. A major problem has been to find the algebraic structure of the stable operations in these theories analogous to the Steenrod algebra in ordinary cohomology, and to apply the results to generalised characteristic classes of manifolds. Attention is restricted in the next section to just one of these cohomology theories, which is of relevance to later sections of this paper.

1.2 Algebras related to the Steenrod algebra. Milnor [132] introduced the complex analogue $\mathbf{M U}^{*}$ of Thom's bordism theory. The stable operations $\mathbf{M U}^{*}(M U)$ were worked out by Landweber [111] and Novikov [149]. Briefly, $\mathbf{M U}^{*}(M U)$ can be identified additively with $\mathbf{M U}^{*}(\mathrm{pt}) \otimes \mathscr{S}^{*}$, where $\mathbf{M} \mathbf{U}^{*}(\mathrm{pt})$ is the value of $\mathbf{M U}^{*}$ on a point, and $\mathscr{S}^{*}$ is the Landweber-Novikov algebra. The multiplicative structure of $\mathbf{M U}^{*}(M U)$ is complicated, but the Landweber-Novikov algebra itself is a Hopf 
algebra which admits an algebraic description in a way analogous to the description of the Steenrod algebra in terms of the Milnor basis. The dual, $\mathscr{S}_{*}$, of $\mathscr{S}^{*}$ is a polynomial algebra $\mathbf{Z}\left[b_{1}, b_{2}, \ldots, b_{n}, \ldots\right]$ with generators $b_{n}$ in grading $n$. (Normally, in the topology literature, $b_{n}$ appears in grading $2 n$.) Since $\mathscr{S}^{*}$ is a Hopf algebra, the coproduct is determined by its value on the generators $b_{n}$. Consider the formal power series

$$
b(t)=b_{0}+b_{1} t+b_{2} t^{2}+\cdots+b_{n} t^{n}+\cdots,
$$

where $t$ is an indeterminate and $b_{0}=1$. Let $v(r, s)$ denote the coefficient of $t^{r}$ in the expansion of $b(t)^{s}$.

Definition 1.13. The coproduct in $\mathscr{S}^{*}$ is given by

$$
\psi\left(b_{n}\right)=\sum_{0 \leqslant j \leqslant n} v(n-j, j+1) \otimes b_{j} .
$$

This coproduct is related to functional substitution of power series. Indeed, the conjugate of $b_{k}$ is the coefficient of $t^{k+1}$ in the functional inverse of $t b(t)$, which can be found from Lagrange's reversion formula $[47,77,80,163,164]$.

Additively, $\mathscr{S}^{*}$ is generated over the integers by the duals of the monomials in the polynomial algebra $\mathbf{Z}\left[b_{1}, b_{2}, \ldots, b_{n}, \ldots\right]$. The sequence notation $s\left(r_{1}, r_{2}, \ldots\right)$ is used in the topology literature to denote the dual of the monomial $b_{1}^{r_{1}} b_{2}^{r_{2}} \cdots$. In his original paper on the subject, Landweber [111] proves a number of relations between composites of the additive generators $s\left(r_{1}, r_{2}, \ldots\right)$, establishes certain minimal algebraic generating sets of the reductions of $\mathscr{S}^{*}$ modulo primes, and identifies the algebra of modulo $p$ Steenrod operations inside such a reduction. In Sections 4 and 5 of [149], Novikov expresses the Steenrod operations and MU operations by their action on polynomials, and ties in the theory with symmetric functions, Stiefel-Whitney classes, Chern classes and Thom isomorphisms. In [102], Kozma extends Landweber's relations, and solves the problem of finding the irreducibles in the Landweber-Novikov algebra over the integers. The same results appear in Aikawa's paper [14], where an explicit product formula is developed for the additive generators. Recently, a natural product formula has been given in terms of composition of differential operators [221].

1.3 Remarks. A steady stream of work on the internal structure of the Steenrod algebra and its action on polynomials has continued to the present time. For example, Adams and Margolis [8] classify Hopf subalgebras of $\mathscr{A}$. Frank Peterson's work $[156,158,159]$ on the action of the Steenrod algebra on the cohomology of projective spaces has sparked off a general interest in hit problems. Questions about excess and conjugation in $\mathscr{A}$ have been investigated notably by Don Davis [55] and others $[\mathbf{2 0}, \mathbf{2 1}, \mathbf{7 0}, \mathbf{1 0 3}, \mathbf{1 1 6}, \mathbf{1 9 1}]$. Recent advances in these areas can be traced through the work of Crabb, Crossley and Hubbuck $[49$, 50, 51, 52, 53], Ken Monks $[139,138,142,143,140]$, and Bill Singer and Judith Silverman $[174,175,178,182]$.

In [36], Bullett and Macdonald devise a method for generating the Adem relations by equating coefficients in certain products of formal power series. This global approach to the study of squaring operations via generating functions emerges in the paper of Atiyah and Hirzebruch [19], and is treated more extensively in Li's work [116]. The global approach is now usually adopted in setting up the Steenrod 
algebra in an algebraic context. For example, in Larry Smith's book [183] and Kuhn's work [107, 108, 109], the rules in Lemma 1.3 are extended to cover finite fields through generating functions for total operations. Let $\mathbf{F}_{q}$ denote the Galois field, where $q$ is a power of the prime $p$. The Steenrod reduced power operations are linear transformations

$$
P^{n}: \mathbf{W}_{d} \otimes \mathbf{F}_{q} \rightarrow \mathbf{W}_{d+n(q-1)} \otimes \mathbf{F}_{q},
$$

uniquely characterised by the following rules satisfied by the formal power series

$$
P(t)=P^{0}+P^{1} t+\cdots+P^{n} t^{n}+\cdots
$$

in the indeterminate $t$.

Lemma 1.14.

$$
P(t): \mathbf{W} \otimes \mathbf{F}_{q} \rightarrow \mathbf{W} \otimes \mathbf{F}_{q}[[t]]
$$

is a ring homomorphism satisfying the normalising conditions

$$
P(t)(1)=1, \quad P(t)(x)=x+x^{q} t,
$$

for $x$ of degree 1 .

It should be noted here that the algebraic rather than the topological conventions have been adopted for grading in the odd prime case. Putting $q=2$ then reproduces the rules in Lemma 1.3 for $P^{n}=S q^{n}$.

The Cartan formulae from Theorem 1.1,

$$
P^{n}(f g)=\sum_{0 \leqslant r \leqslant n} P^{r}(f) P^{n-r}(g),
$$

are obtained by picking off the coefficient of $t^{n}$ in the expansion of $P(t)(f g)$.

It is then possible to verify the normalisation conditions $P^{n}(f)=f^{q}$ if $\operatorname{deg} f=n$, and $P^{n}(f)=0$ if $\operatorname{deg} f<n$, and, for $x$ of degree 1 , the formula

$$
P^{n}\left(x^{k}\right)=\left(\begin{array}{l}
k \\
n
\end{array}\right) x^{k+n(q-1)} .
$$

Smith explains in Chapters 10 and 11 of his book [183] how to obtain the BullettMacdonald formula.

Theorem 1.15 (Bullett and Macdonald [36]).

$$
P(s) P(1)=P(u) P\left(t^{q}\right),
$$

where $u=1+t+\cdots+t^{(q-1)}$ and $s=t u$.

The Adem relations in the $q$-case can then be derived as in [36]:

$$
P^{i} P^{j}=\sum_{0 \leqslant k \leqslant[i / q]}(-1)^{(i+1)}\left(\begin{array}{c}
(q-1)(j-k)-1 \\
i-q k
\end{array}\right) P^{i+j-k} P^{k}
$$

for $0<i<q j$, where the binomial coefficients are taken modulo $p$.

There are a number of other methods for setting up Steenrod operations in various contexts that we shall mention briefly. Early work on the geometric approach to Steenrod squares relates to Smith theory $[\mathbf{2 9}, \mathbf{2 0 0}, \mathbf{2 2 5}$, double point sets in 
bordism with singularities, and Spanier-Whitehead duality [130]. Dold sets up the Steenrod algebra for cohomology of a topological space in [60]. Quillen [161] uses Steenrod operations in bordism, further developed by Tom Dieck [202], in connection with formal groups and the Lazard ring. In [25], Bisson and Joyal treat similar topics in terms of a divided differential theory of coverings. In $[\mathbf{9 5}, \mathbf{9 6}]$, Karoubi combines the de Rham theory of differential forms for the homology of manifolds with symmetric products of topological spaces, to obtain certain chain complexes in terms of which cohomology of a space and Steenrod operations can be defined. In a more algebraic setting, Benson [23] shows how to set up the Steenrod algebra in the context of group cohomology theory. In the area of algebraic groups, Kaneda, Shimada, Tezuka and Yagita [93] represent the Steenrod algebra in terms of distributions on the unipotent subgroups of general linear groups. In the realm of invariant theory of general linear groups over finite fields, Mui [146] identifies the Milnor basis elements in terms of Dickson invariants under a certain coaction of the cohomology of elementary 2-groups. Recent work in this direction can be found in [118]. Lomonaco [119] has further related the Dickson invariants to the DyerLashof algebra in terms of May's universal Steenrod algebra [128] and the lambda algebra, which is a powerful device for calculating the cohomology of the Steenrod algebra [180] (not to be confused with the algebra $\Lambda$ of symmetric functions, to be discussed in a later section). We refer to Pengelley and Williams [155] for some new developments.

\section{Differential operators and integral Steenrod squares}

The appropriate setting for integral Steenrod squares is complex bordism theory, where the Landweber-Novikov algebra plays the role of the Steenrod algebra.

2.1 The divided differential operator algebra. The construction of the divided differential operator algebra is explained in [221]. The main features are summarised in the following paragraphs.

Let

$$
D_{k}=\sum_{i \geqslant 1} x_{i}^{k+1} \frac{\partial}{\partial x_{i}},
$$

for $k \geqslant 1$, be the partial differential operator acting in the usual way on the integral polynomial ring $\mathbf{W}=\mathbf{Z}\left[x_{1}, \ldots, x_{n}, \ldots\right]$. Although $D_{k}$ is formally an infinite sum, its action on a polynomial involves only a finite number of variables in any instance. The restriction of $D_{k}$ to $\mathbf{W}(n)$ is an element of the Weyl algebra in $n$ variables [221]. Following standard methods for relating filtered algebras to graded algebras, the wedge product $\vee$ of two differential operators, with variable coefficients, is defined by allowing the derivatives of the first operator to pass the variable coefficients of the second operator without acting. The wedge product is commutative and gives the term of highest differential order in the composition of the operators. Normally, juxtaposition of operators denotes composition, but occasionally for emphasis we shall use the symbol $\circ$. For example, the composite $D_{1} \circ D_{1}$ is given by

$$
\left(\sum_{i \geqslant 1} x_{i}^{2} \frac{\partial}{\partial x_{i}}\right)\left(\sum_{i \geqslant 1} x_{i}^{2} \frac{\partial}{\partial x_{i}}\right)=2\left(\sum_{i \geqslant 1} x_{i}^{3} \frac{\partial}{\partial x_{i}}\right)+\sum_{\left(i_{1}, i_{2}\right)} x_{i_{1}}^{2} x_{i_{2}}^{2} \frac{\partial^{2}}{\partial x_{i_{1}} \partial x_{i_{2}}},
$$


where the last summation is taken over all 2-vectors of non-negative integers $\left(i_{1}, i_{2}\right)$. Hence $D_{1} \circ D_{1}=2 D_{2}+D_{1} \vee D_{1}$. It should be noted that $D_{1} \vee D_{1}$ is divisible by 2 as an integral operator. More generally, an iterated wedge product is given by the formula

$$
D_{k_{1}} \vee D_{k_{2}} \vee \cdots \vee D_{k_{r}}=\sum_{\left(i_{1}, \ldots, i_{r}\right)} x_{i_{1}}^{k_{1}+1} \cdots x_{i_{r}}^{k_{r}+1} \frac{\partial^{r}}{\partial x_{i_{1}} \cdots \partial x_{i_{r}}},
$$

where the summation is taken over all $r$-vectors of non-negative integers. It can be seen from this that the iterated wedge product $D_{k}^{\vee r}$ is divisible by $r$ ! as an integral operator. By definition, the divided differential operator algebra $\mathscr{D}$ is generated over the integers by the divided operators $D_{k}^{\vee r} / r$ ! under wedge product.

Let $K=k_{1}^{r_{1}} k_{2}^{r_{2}} \cdots k_{a}^{r_{a}}$ denote a multiset of positive integers $k_{i}$ with positive superscripts $r_{i}$. Let $\left(t_{1}, t_{2}, \ldots\right)$ be a sequence of commuting indeterminates $t_{i}$. The usual abbreviated notation $t^{K}=t_{k_{1}}^{r_{1}} t_{k_{2}}^{r_{2}} \cdots t_{k_{a}}^{r_{a}}$ is adopted for a monomial in the $t_{i}$, and

$$
D(K)=\frac{D_{k_{1}}^{\vee r_{1}}}{r_{1} !} \vee \frac{D_{k_{2}}^{\vee r_{2}}}{r_{2} !} \vee \cdots \vee \frac{D_{k_{a}}^{\vee r_{a}}}{r_{a} !}
$$

denotes the iterated wedge product of divided differential operators. For example,

$$
D(k)=D_{k}, \quad D\left(k^{r}\right)=\frac{D_{k}^{\vee r}}{r !} .
$$

It should be noted that $D\left(k_{1}^{r_{1}} k_{2}^{r_{2}}\right)=D\left(k_{1}^{r_{1}}\right) \vee D\left(k_{2}^{r_{2}}\right)$. In particular, when $k_{1}=k_{2}=k$, we have

$$
D\left(k^{r_{1}} k^{r_{2}}\right)=D\left(k^{r_{1}}\right) \vee D\left(k^{r_{2}}\right)=\frac{\left(r_{1}+r_{2}\right) !}{r_{1} ! r_{2} !} D\left(k^{r_{1}+r_{2}}\right),
$$

indicating that $k^{r_{1}} k^{r_{2}}$ and $k^{r_{1}+r_{2}}$ are different multisets.

NotE. The use of superscripts in multiset notation can lead to confusion unless care is taken with the exponential law. To explain this in a general context, let $C$ denote a set of mathematical entities, and consider vectors $\left(c_{1}, \ldots, c_{n}\right)$ of elements $c_{i}$ in $C$. Two such vectors are equivalent if they have the same coordinates up to permutation. An equivalence class is what is meant by a (finite) multiset of elements of $C$. Such a multiset [13] is determined by a function $\pi: C \rightarrow\{0\} \cup \mathbf{N}$ which takes the value zero on all but a finite number of members of $C$. The function $\pi$ measures the number of repetitions of an element. A multiset may be written as an associative, commutative formal string of elements of $C$, allowing repetitions, but not allowing the unqualified exponential notation for repeated elements. The reason is that, in some contexts where double indexing is needed, the notation $c^{r}$ is reserved for an element in the cartesian product $C \times \mathbf{N}$, and multisets are taken over this set rather than $C$ itself. In this case, for example, $c^{1} c^{1}$ and $c^{2}$ are different multisets of elements from $C \times \mathbf{N}$ and, strictly speaking, must further be distinguished from $c c$, which is a multiset of elements in $C$. Of course, it is standard practice to identify $C$ with $C \times\{1\}$ in $C \times \mathbf{N}$ and view $c$ as the same as $c^{1}$. Concatenation of formal strings of elements of $C$ gives rise to the polynomial algebra $\mathscr{P}(C)$ generated over the integers by the set $C$, but it might be safer in the present context to use the wedge symbol $\vee$ for the product in this algebra. The superscript notation $c^{\vee r}$ then means the $r$-fold concatenation of the element $c$. We shall nevertheless continue to use the term 'multiset' to refer to $K=k_{1}^{r_{1}} k_{2}^{r_{2}} \cdots k_{a}^{r_{a}}$ with distinct $k_{i}$, unless otherwise 
specified. Conventionally, a zero exponent $r=0$ indicates the absence of the factor $k^{r}$ in the multiset. For example, $D\left(k^{0}\right)$ is identified with the identity element.

The collection $D(K)$, as $K$ ranges over multisets, forms an additive basis for $\mathscr{D}$. A significant fact is that $\mathscr{D}$ is closed under composition of operators. Furthermore, the natural coproduct $\psi\left(D_{n}\right)=1 \otimes D_{n}+D_{n} \otimes 1$ makes $\mathscr{D}$ into a Hopf algebra with respect to both the composition and the wedge products. Under composition, $\mathscr{D}$ is isomorphic to the Landweber-Novikov algebra [221]. The operators $D_{n}$ are the primitives of the Hopf algebra.

There is a Leibniz formula for the wedge-iterated differentiation of a product of polynomials $f, g$, which can best be expressed in terms of multisets by means of a generating function for the basis elements $D(K)$. This is provided by the wedge exponential function of a differential operator $E$,

$$
\exp _{\vee}(E)=1+E+\frac{E^{\vee 2}}{2 !}+\cdots+\frac{E^{\vee n}}{n !}+\cdots .
$$

Let $\mathbf{t}=\left(t_{1}, t_{2}, \ldots\right)$ be a formal sequence of commuting indeterminates, and let $\mathbf{D}=\left(D_{1}, D_{2}, \ldots\right)$ denote the sequence of differential operators. Now introduce the formal inner product

$$
\langle\mathbf{t}, \mathbf{D}\rangle=t_{1} D_{1}+t_{2} D_{2}+\cdots .
$$

Using multiset notation and the commutativity of the wedge product, we obtain

$$
\begin{aligned}
\exp _{\vee}(\langle\mathbf{t}, \mathbf{D}\rangle) & =\exp _{\vee}\left(t_{1} D_{1}+t_{2} D_{2}+\cdots\right) \\
& =\exp _{\vee}\left(t_{1} D_{1}\right) \vee \exp _{\vee}\left(t_{2} D_{2}\right) \vee \cdots \vee \exp _{\vee}\left(t_{n} D_{n}\right) \vee \cdots \\
& =\sum_{k \geqslant 0} t_{1}^{k} D\left(1^{k}\right) \vee \sum_{k \geqslant 0} t_{2}^{k} D\left(2^{k}\right) \vee \cdots \vee \sum_{k \geqslant 0} t_{n}^{k} D\left(n^{k}\right) \vee \cdots \\
& =\sum_{K} t^{K} D(K),
\end{aligned}
$$

where the final sum is taken over all multisets $K$. Then the Leibniz formula for differentiating a product of polynomials $f, g$ is as follows.

LEMMA 2.1.

$$
\exp _{\vee}(\langle\mathbf{t}, \mathbf{D}\rangle)(f g)=\left(\exp _{\vee}(\langle\mathbf{t}, \mathbf{D}\rangle)(f)\right)\left(\exp _{\vee}(\langle\mathbf{t}, \mathbf{D}\rangle)(g)\right) .
$$

Furthermore, since all derivatives of order greater than 1 vanish on the variable $x$ in degree 1, we also have the normalising formula

$$
\exp _{\vee}(\langle\mathbf{t}, \mathbf{D}\rangle)(x)=x\left(1+t_{1} x+t_{2} x^{2}+\cdots\right) .
$$

Lemma 2.1 furnishes an integral version of the rules of Lemmas 1.14 and 1.3, and converts the Leibniz formulae into the Cartan formulae.

We recall from [221] the definition of the integral Steenrod squares $S Q^{r}=D\left(1^{r}\right)$. It is shown in [221] that the modulo 2 reduction of $S Q^{k}$ is $S q^{k}$. More generally, the Milnor basis elements are given as follows.

Lemma 2.2. After modulo 2 reduction,

$$
S q\left(r_{1}, r_{2}, \ldots, r_{a}\right)=D\left(1^{r_{1}} 3^{r_{2}} \cdots\left(2^{a}-1\right)^{r_{a}}\right) .
$$


The coproduct of integral squares is given by

$$
\psi\left(S Q^{k}\right)=\sum_{0 \leqslant i \leqslant k} S Q^{i} \otimes S Q^{k-i} .
$$

It follows that the subalgebra $\mathscr{N}$ of $\mathscr{D}$ generated under composition by the integral Steenrod squares is a Hopf subalgebra and reduces modulo 2 to the Steenrod algebra. Similar results apply to the odd prime situation, by considering the differential operators $D_{p-1}$ and $D_{p^{r}-1}$ in place of $D_{1}$ and $D_{2^{r}-1}$. These are Landweber's original results [111] interpreted in terms of differential operators.

The Lie bracket of $D_{k}$ and $D_{l}$ is defined in the usual way by $\left[D_{k}, D_{l}\right]=D_{k} D_{l}-$ $D_{l} D_{k}$, and the following lemma is immediate from the definition of the differential operators.

$$
\text { Lemma 2.3. }\left[D_{k}, D_{l}\right]=(l-k) D_{k+l} \text {. }
$$

The additive group generated by the $D_{k}$ is therefore a Lie algebra, and its universal enveloping algebra $\mathscr{U}$ is the associative algebra generated by the $D_{k}$ under composition. Hence $\mathscr{U}$ is a subalgebra of $\mathscr{D}$.

The following reduction formulae relating the integral squaring operators to the $D_{k}$ were established in Lemma 5.6 of $[\mathbf{2 2 1}]$.

LeMma 2.4. $r S Q^{r}=D_{1} S Q^{r-1}-2 D_{2} S Q^{r-2}+2^{2} D_{3} S Q^{r-3}-\cdots \pm 2^{r-1} D_{r}$.

The proof goes through for any integer $q$ greater than 1, and gives

$$
\begin{aligned}
r D\left((q-1)^{r}\right)= & D(q-1) D\left((q-1)^{r-1}\right)+\cdots \\
& +(-1)^{k-1} q^{k-1} D(k(q-1)) D\left((q-1)^{r-k}\right)+\cdots \pm q^{r-1} D(r(q-1)) .
\end{aligned}
$$

THEOREM 2.5 (Wood [221]). Over the rationals, the divided differential operator algebra $\mathscr{D}$, the universal enveloping algebra $\mathscr{U}$, and the integral Steenrod algebra $\mathscr{N}$ are equal, and each is generated by $S Q^{1}$ and $S Q^{2}$ under composition.

Equivalently, the differential operator algebra is generated over the rationals by $D_{1}$ and $D_{2}$. The proof is a straightforward application of Lemmas 2.3 and 2.4.

Product formulae for basis elements in the Landweber-Novikov algebra were established in Landweber's original paper [111] and extended in the papers of Aikawa [14] and Kozma [102]. The following application is quoted here in the language of differential operators and multisets.

THEOREM 2.6 (Kozma [102]). The elements $D\left(1^{p^{n}}\right), D\left(2^{p^{n}}\right)$, as $p$ ranges over primes and $n \geqslant 0$, form a minimal algebraic generating set for $\mathscr{D}$.

More details on relations between these generators can be found in [102].

Richard Kane [92] has produced analogues of the Milnor product formulae in $B P$-theory. $\mathrm{Li}[\mathbf{1 1 5}, \mathbf{1 1 6}]$ has constructed global product formulae for certain types of Hopf algebras in terms of the dual coproduct and convolution of formal sequences. Total Steenrod squaring operations are studied in $[\mathbf{1 0 0}, \mathbf{1 2 0}]$. Aikawa's product formula [14] for the Landweber-Novikov algebra is phrased in the traditional sequence notation $s\left(r_{1}, r_{2}, \ldots\right)$. In [221], the product formula in the differential 
operator algebra is phrased in terms of multisets. Both are complicated in their most general form. Theorem 4.4 of [221] presents a more workable version of the product formula, which we quote here. It applies in the first instance to simple sets $K=\left\{k_{1}, \ldots, k_{a}\right\}, L=\left\{l_{1}, \ldots, l_{b}\right\}$ of positive numbers. Let $L$ be augmented by an extra element $l_{0}=0$, to make the set $L^{+}=\left\{l_{0}, l_{1}, \ldots, l_{b}\right\}=L \cup\left\{l_{0}\right\}$. Consider a set function $\Phi: K \rightarrow L^{+}$. Combinatorial functions are associated with $\Phi$ in the following way:

$$
\tau\left(l_{i}\right)=\#\left\{\Phi^{-1} l_{i}\right\}, \quad \sigma\left(l_{i}\right)=\sum_{k_{j} \in \Phi^{-1} l_{i}} k_{j}, \quad \rho\left(l_{i}\right)=\left(l_{i}+1\right)\left(l_{i}\right) \cdots\left(l_{i}+2-\tau\left(l_{i}\right)\right),
$$

measuring, respectively, the number of elements in a fibre of the function, the sum of the elements in a fibre, and the falling factorial associated with an element of $L$. We adopt the conventions $\rho\left(l_{i}\right)=1$ if $\tau\left(l_{i}\right)=0$, and write $\rho(\Phi)=\rho\left(l_{1}\right) \rho\left(l_{2}\right) \cdots \rho\left(l_{b}\right)$. These are combinatorial ingredients which one would expect to enter a formula for composing two differential operators with variable coefficients.

TheOrem 2.7 (Wood [221]).

$$
D(K) \circ D(L)=\sum_{\Phi} \rho(\Phi) D\left(\Phi^{-1}\left(l_{0}\right)\right) \vee D_{l_{1}+\sigma\left(l_{1}\right)} \vee D_{l_{2}+\sigma\left(l_{2}\right)} \vee \cdots \vee D_{l_{b}+\sigma\left(l_{b}\right)},
$$

where the summation is taken over all functions

$$
\Phi:\left\{k_{1}, k_{2}, \ldots, k_{a}\right\} \rightarrow\left\{l_{0}, l_{1}, l_{2}, \ldots, l_{b}\right\} .
$$

The following special cases are typical of the formulae used by Landweber, Kozma and Aikawa in arriving at the irreducibles in the Landweber-Novikov algebra.

EXAMPLE 2.8. Taking $K=\{k\}$ to have one element, we have

$$
\begin{gathered}
D_{k} \circ\left(D_{l_{1}} \vee \cdots \vee D_{l_{b}}\right) \\
=D_{k} \vee D_{l_{1}} \vee \cdots \vee D_{l_{b}}+\left(l_{1}+1\right) D_{k+l_{1}} \vee \cdots \vee D_{l_{b}}+\cdots+\left(l_{b}+1\right) D_{l_{1}} \vee \cdots \vee D_{k+l_{b}} .
\end{gathered}
$$

In this formula, $k$ is added to each subscript in turn on the right-hand side of the equality.

In the case where $L$ has just one element, a function from $K$ into $L^{+}=\left\{l_{0}, l\right\}$ may be viewed as an ordered partition of the set $K=\left\{k_{1}, \ldots, k_{a}\right\}$ into two disjoint subsets $U=\left\{u_{1}, \ldots, u_{r}\right\}$ and $V=\left\{v_{1}, \ldots, v_{s}\right\}$, where $r+s=a$, and conventionally $r=0$ or $s=0$ indicates that the corresponding set is empty. For such a partition, let $\rho(r)=(l+1)(l) \cdots(l+2-r)$, where conventionally $\rho(0)=1$. Then the product formula yields the following.

EXAMPLE 2.9.

$$
\left(D_{k_{1}} \vee \cdots \vee D_{k_{a}}\right) \circ D_{l}=\sum_{(U, V)} \rho(s) D_{u_{1}} \vee \cdots \vee D_{u_{r}} \vee D_{l+v_{1}+\cdots+v_{s}},
$$

where the sum is taken over the ordered partitions $U, V$ of $K$ into two subsets $U=\left\{u_{1}, \ldots, u_{r}\right\}, V=\left\{v_{1}, \ldots, v_{s}\right\}$.

As an application of the general product formula, Theorem 4.2 in [221], consider 
the case where $K=k^{r}$ and $L=l^{s}$. Let $\Theta$ stand for a solution of the simultaneous equations

$$
s=t_{0}+\sum_{1 \leqslant i \leqslant l+1} t_{i}, \quad r=\theta_{0}+\sum_{1 \leqslant i \leqslant l+1} i t_{i},
$$

in non-negative integers $\theta_{0}, t_{i}$, and set $\rho(\Theta)=\prod_{i=1}^{l+1}\left(\begin{array}{c}l+1 \\ i\end{array}\right)^{t_{i}}$.

EXAMPLE 2.10.

$$
D\left(k^{r}\right) \circ D\left(l^{s}\right)=\sum_{\Theta} \rho(\Theta) D\left(k^{\theta_{0}}\right) \vee \bigvee_{j=0}^{j=l+1} D\left((l+j k)^{t_{j}}\right),
$$

where the sum is taken over all solutions $\Theta$ of the given equations.

Since no two of the numbers $l+j k$ can be equal for different values of $j$, we can write this formula, in case $k \neq l$, more succinctly as

$$
D\left(k^{r}\right) \circ D\left(l^{s}\right)=\sum_{\Theta} \rho(\Theta) D\left(k^{\theta_{0}} l^{t_{0}}(l+k)^{t_{1}} \cdots(l+(l+1) k)^{t_{l+1}}\right) .
$$

If $k=l$, then the expression has to be modified to

$$
D\left(k^{r}\right) \circ D\left(k^{s}\right)=\sum_{\Theta} \rho(\Theta) \frac{\left(\theta_{0}+t_{0}\right) !}{\theta_{0} ! t_{0} !} D\left(k^{\theta_{0}+t_{0}}(2 k)^{t_{1}} \cdots\left(k^{2}+2 k\right)^{t_{k+1}}\right) .
$$

Specialising to the cases where the entries in $L$ and $K$ are equal to 1 or 2 gives rise to the following identities.

EXAMPLE 2.11.

$$
\begin{aligned}
S Q^{1} \circ S Q^{1} & =2 D_{2}+2 S Q^{2}, \\
S Q^{1} \circ S Q^{2} & =2 D_{1} \vee D_{2}+3 S Q^{3}, \\
S Q^{2} \circ S Q^{1} & =D_{3}+2 D_{1} \vee D_{2}+3 S Q^{3}, \\
S Q^{1} \circ S Q^{1} \circ S Q^{1} & =6\left(D_{1} \vee D_{2}+D_{3}+S Q^{3}\right), \\
D_{3} & =\left[S Q^{2}, S Q^{1}\right] .
\end{aligned}
$$

From these formulae we can obtain the first relation in the integral Steenrod algebra $\mathscr{N}$ which occurs in grading 3.

EXAMPLe 2.12. $S Q^{1} S Q^{1} S Q^{1}=6\left[S Q^{2}, S Q^{1}\right]+3\left(S Q^{1} S Q^{2}-S Q^{3}\right)$.

A basis for $\mathscr{N}$ up to grading 3 is given by

$$
S Q^{1}, \quad S Q^{2}, \quad S Q^{1} S Q^{1}, \quad S Q^{3}, \quad S Q^{2} S Q^{1}, \quad S Q^{1} S Q^{2} .
$$

We can ask for a basis of $\mathscr{N}$ in all gradings. A rational basis for $\mathscr{N}$, which is the same as $\mathscr{D}$ and $\mathscr{U}$ over $\mathbf{Q}$ by Theorem 2.5, is given by the classical PoincaréBirkhoff-Witt theorem in the universal enveloping algebra of a Lie algebra [89]. The set of composites $D_{k_{1}}^{\circ r_{1}} \circ D_{k_{2}}^{\circ r_{2}} \circ \cdots \circ D_{k_{a}}^{\circ r_{a}}$, where $k_{1}>k_{2}>\ldots>k_{a}$, forms an additive basis of $\mathscr{U}$. It is not immediately clear that a similar statement is true if $S Q^{k}$ is substituted for $D_{k}$. In any case, we see from the relations exhibited above that

$$
S Q^{3}, \quad S Q^{2} S Q^{1}, \quad S Q^{1} S Q^{1} S Q^{1}
$$


is definitely not a basis of $\mathscr{N}$ in grading 3 , and therefore we cannot expect to parametrise a basis for $\mathscr{N}$ in terms of composites of integral Steenrod squares by partitions of the grading in descending order of parts.

2.2 Global product formulae. We shall now look at a global formulation of the product formulae in the differential operator algebra, following the work of Li [116]. Consider the polynomial algebra $\mathbf{Z}\left[b_{1}, b_{2}, \ldots\right]$ equipped with the Landweber-Novikov coproduct as explained in Definition 1.13, namely

$$
\psi\left(b_{n}\right)=\sum_{0 \leqslant j \leqslant n} v(n-j, j+1) \otimes b_{j},
$$

where $v(r, s)$ denotes the coefficient of $t^{r}$ in the expansion of $\left(1+b_{1} t+b_{2} t^{2}+\cdots\right)^{s}$.

EXAMPLE 2.13.

$$
\psi\left(b_{1}\right)=b_{1} \otimes b_{0}+b_{0} \otimes b_{1}, \quad \psi\left(b_{2}\right)=b_{2} \otimes b_{0}+2 b_{1} \otimes b_{1}+b_{0} \otimes b_{2} .
$$

Let $\mathbf{t}=\left(t_{1}, t_{2}, \ldots\right), \mathbf{s}=\left(s_{1}, s_{2}, \ldots\right)$ denote two sequences of indeterminates associated with the power series

$$
\mathbf{t}(x)=x+t_{1} x^{2}+\cdots+t_{n} x^{n+1}+\cdots, \quad \mathbf{s}(x)=x+s_{1} x^{2}+\cdots+s_{n} x^{n+1}+\cdots,
$$

and define the convolution product $\mathbf{t} * \mathbf{s}$ to be the sequence associated with the power series

$$
\mathbf{u}(x)=x+u_{1} x^{2}+\cdots+u_{n} x^{n+1}+\cdots,
$$

where $u_{n}=(\mathbf{t} * \mathbf{s})_{n}$, obtained by substituting $\mathbf{t}$ in $\mathbf{s}$; in other words, $\mathbf{u}(x)$ is the composite power series $\mathbf{s}(\mathbf{t}(x))$.

EXAMPLE 2.14. The first few terms of the convolution product $(\mathbf{t} * \mathbf{s})$ are

$$
\left(t_{1}+s_{1}, t_{2}+2 t_{1} s_{1}+s_{2}, t_{3}+s_{1}\left(t_{1}^{2}+2 t_{2}\right)+3 t_{1} s_{2}+s_{3}, \ldots\right) .
$$

In general, the coefficient $u_{n}$ is related to the partial Bell polynomial [121] by replacing $t_{i}$, $s_{i}$ with $t_{i} /(i+1) !, s_{i} /(i+1)$ !, respectively, and multiplying by $(n+1)$ ! to restore integer coefficients.

The convolution product of formal sequences is associative but not commutative, and the convolution inverse $\hat{\mathbf{t}}$, satisfying $\hat{\mathbf{t}} * \mathbf{t}=0$, is obtained from the composition inverse of the power series $\mathbf{t}(x)$. This is given by the Lagrange reversion formula, as follows.

LEMMA 2.15.

$$
\hat{\mathbf{t}}(t)=x+\frac{v_{1}}{2} x^{2}+\cdots+\frac{v_{n}}{n+1} x^{n+1}+\cdots,
$$

where $v_{n}$ is the coefficient of $x^{n}$ in the expansion of

$$
\left(1+t_{1} x+t_{2} x^{2}+\cdots\right)^{-n-1} .
$$

EXAMPLE 2.16. The first few terms of the convolution inverse of $\mathbf{t}$ are

$$
\left(-t_{1},-t_{2}+2 t_{1}^{2},-t_{3}+5 t_{1} t_{2}-3 t_{1}^{3}, \ldots\right) \text {. }
$$


Now recall the inner product $\langle\mathbf{t}, \mathbf{D}\rangle=t_{1} D_{1}+t_{2} D_{2}+\cdots$ and the wedge exponential function $\exp _{\mathrm{v}}(\langle\mathbf{t}, \mathbf{D}\rangle)=\sum_{K} t^{K} D(K)$. The following theorem encapsulates the global structure of the differential operator algebra.

THEOREM 2.17 (Wood [221]). The product, coproduct and conjugation in the differential operator algebra $\mathscr{D}$ are given globally by

$$
\begin{aligned}
& \exp _{\vee}(\langle\mathbf{t}, \mathbf{D}\rangle) \circ \exp _{\vee}(\langle\mathbf{s}, \mathbf{D}\rangle)=\exp _{\vee}(\langle\mathbf{t} * \mathbf{s}, \mathbf{D}\rangle), \\
& \psi\left(\exp _{\vee}(\langle\mathbf{t}, \mathbf{D}\rangle)\right)=\exp _{\vee}(\langle\mathbf{t}, \mathbf{D}\rangle) \otimes \exp _{\vee}(\langle\mathbf{t}, \mathbf{D}\rangle), \\
& \chi\left(\exp _{\vee}(\langle\mathbf{t}, \mathbf{D}\rangle)\right)=\exp _{\vee}(\langle\hat{\mathbf{t}}, \mathbf{D}\rangle) .
\end{aligned}
$$

The first formula states that the composite $D(K) \circ D(L)$ is the coefficient of $t^{K} s^{L}$ in the exponential expansion of the convolution product. The second formula is the Leibniz formula of Lemma 2.1, expressed in terms of the coproduct. The third formula states that the conjugate $\chi(D(K))$ is the coefficient of $t^{K}$ in the exponential expansion of the convolution inverse. Putting $s=\hat{t}$ in the product formula gives the result

$$
\sum_{K} t^{K} D(K) \circ \sum_{L} t^{L} \chi(D(L))=\exp (\langle\hat{t} * t, \mathbf{D}\rangle)=1,
$$

which can be re-stated as follows.

LEMMA 2.18. For any non-empty multiset $M=m_{1}^{t_{1}} \cdots m_{n}^{t_{n}}$, we have

$$
\sum_{K \cup L=M} D(K) \circ \chi(D(L)=0
$$

where the summation is over all ordered pairs of multisets

$$
K=m_{1}^{r_{1}} \cdots m_{n}^{r_{n}}, \quad L=m_{1}^{s_{1}} \cdots m_{n}^{s_{n}}
$$

such that $r_{i}+s_{i}=t_{i}$ for $1 \leqslant i \leqslant n$.

Similarly, the coproduct formula can be written in the form of a generalised Leibniz formula.

Lemma 2.19. For any non-empty multiset $M=m_{1}^{t_{1}} \cdots m_{n}^{t_{n}}$, and polynomials $u, v$, we have

$$
D(M)(u v)=\sum_{K \cup L=M} D(K)(u) D(L)(v),
$$

where the summation is over all pairs of multisets

$$
K=m_{1}^{r_{1}} \cdots m_{n}^{r_{n}}, \quad L=m_{1}^{s_{1}} \cdots m_{n}^{s_{n}}
$$

such that $r_{i}+s_{i}=t_{i}$ for $1 \leqslant i \leqslant n$.

Taking $M=1^{k}, k>1$, in Lemma 2.18 , we obtain

$$
\sum_{0 \leqslant i \leqslant k} S Q^{i} \chi\left(S Q^{k-i}\right)=0
$$

whose modulo 2 reduction is a familiar formula in the Steenrod algebra.

The above formulae will prove useful in the later section on hit problems. 
Note. Theorem 2.17 is essentially a transcription of Li's Theorem 3.2 in [116]. We refer to Li's star operator on sequences as convolution because the first global structure equation in Theorem 2.17 is reminiscent of the Laplace formula in classical analysis for the convolution product $\mathscr{L}(f * g)=\mathscr{L}(f) \mathscr{L}(g)$, where

$$
\mathscr{L}(f)(s)=\int_{0}^{\infty} e^{-s t} f(t) d t, \quad f * g(t)=\int_{0}^{t} f(s) g(t-s) d s .
$$

The appropriate coproduct in the case of the differential operator algebra is the coproduct of the Landweber-Novikov algebra, whose associated convolution is functional composition of power series.

Although the global formulation of the structure equations for the differential operator algebra is very elegant and provides a framework in which to discuss iterated composites of Steenrod operations [116], in practice it has been found very difficult to get a sufficiently good grip on the divisibility of the coefficients of the exponential expansion in order to apply the results to some of the harder questions about nilpotence in the Steenrod algebra. This matter will be taken up again in a later section.

2.3 The contragredient action of the differential operator algebra. Differential operator algebras arise in the theory of $\mathscr{D}$-modules, with applications in algebraic geometry, quantum groups and Lie algebras [48]. A classic example is the Weyl algebra defined over the integers by generators $x_{1}, \ldots, x_{n}, \partial_{1}, \ldots, \partial_{n}$ subject to relations

$$
\left[x_{i}, x_{j}\right]=0, \quad\left[\partial_{i}, \partial_{j}\right]=0, \quad\left[\partial_{i}, x_{j}\right]=\delta_{i j},
$$

where $\delta_{i j}$ is the Kronecker $\delta$ function. The Weyl algebra is represented by the usual action of $\partial_{i}$ as the partial derivative $\partial / \partial x_{i}$ on polynomials in the $x_{i}$. The differential operator $D_{k}$, restricted to the first $n$ variables, belongs to the Weyl algebra and is a symmetric element under the action of the symmetric group which simultaneously permutes variables and partial derivatives. On the other hand, the divided differential operator $D_{k}^{\vee r} / r$ ! does not, in general, belong to the Weyl algebra over the integers. However, it is clearly necessary to consider such operators in order to have a viable modular theory, such as the Steenrod algebra. A simple illustration of this point is given by the Laplacian

$$
\nabla^{2}=\frac{\partial^{2}}{\partial x_{1}^{2}}+\cdots+\frac{\partial^{2}}{\partial x_{n}^{2}}
$$

whose action on integral polynomials is divisible by 2 . Hence $\nabla^{2} / 2$ is an integral operator, with an interesting modulo 2 reduction. The Weyl algebra contains both 'going down' operators, like the Laplacian, and 'going up' operators, like $D_{k}$. An element $E$ in the Weyl algebra, of one type, is changed into the other type by switching $x_{i}$ and $\partial_{i}$ for each $i$ in the expression for $E$. For example, the going up operator

$$
D_{k}=\sum_{i \geqslant 1} x_{i}^{k+1} \frac{\partial}{\partial x_{i}}
$$

transforms into the going down operator

$$
D_{k}^{*}=\sum_{i \geqslant 1} x_{i} \frac{\partial^{k+1}}{\partial x_{i}^{k+1}} .
$$


This process is sometimes referred to in the literature as the Fourier transform, because in classical analysis, the Fourier transform of the derivative $d f / d x$ of a function $f$ is given by $i x \hat{f}$, where $\hat{f}$ is the Fourier transform of $f$ and $i=\sqrt{-1}$. We can extend the Fourier transform to elements of $\mathscr{D}$, and form the contragredient representation of $\mathscr{D}$ on the dual of the polynomial algebra $\mathbf{W}=\mathbf{Z}\left[x_{1}, \ldots, x_{n}, \ldots\right]$, equipped with its usual coproduct $\psi\left(x_{i}\right)=1 \otimes x_{i}+x_{i} \otimes 1$. Then the dual $\mathbf{W}^{*}$ is the divided polynomial algebra with additive basis

$$
x(K)=\frac{x_{k_{1}}^{r_{1}}}{r_{1} !} \frac{x_{k_{2}}^{r_{2}}}{r_{2} !} \cdots \frac{x_{k_{a}}^{r_{a}}}{r_{a} !}
$$

dual to the monomial base $x^{K}=x_{k_{1}}^{r_{1}} x_{k_{2}}^{r_{2}} \cdots x_{k_{a}}^{r_{a}}$ of $\mathbf{W}$ as $K$ runs over multisets.

EXAmPLE 2.20. The 'going down' integral Steenrod square $S Q_{2}$ is represented by

$$
S Q_{2}=\frac{D_{1}^{*} \vee D_{1}^{*}}{2}=\frac{1}{2} \sum_{(i, j)} x_{i} x_{j} \frac{\partial^{4}}{\partial x_{i}^{2} \partial x_{j}^{2}} .
$$

Its evaluation on divided polynomials in two variables is given by

$$
\begin{aligned}
S Q_{2}\left(\frac{x_{1}^{u}}{u !} \frac{x_{2}^{v}}{v !}\right)= & \frac{1}{2} \sum_{i=1,2} x_{i}^{2} \frac{\partial^{4}}{\partial x_{i}^{4}}\left(\frac{x_{1}^{u}}{u !} \frac{x_{2}^{v}}{v !}\right)+x_{1} x_{2} \frac{\partial^{2}}{\partial x_{1}^{2} \partial x_{2}^{2}}\left(\frac{x_{1}^{u}}{u !} \frac{x_{2}^{v}}{v !}\right) \\
= & \frac{(u-2)(u-3)}{2} \frac{x_{1}^{u-2}}{(u-2) !} \frac{x_{2}^{v}}{v !}+\frac{(v-2)(v-3)}{2} \frac{x_{1}^{u}}{u !} \frac{x_{2}^{v-2}}{(v-2) !} \\
& +(u-1)(v-1) \frac{x_{1}^{u-1}}{(u-1) !} \frac{x_{2}^{v-1}}{(v-1) !} .
\end{aligned}
$$

Checking the equality of the inner products

$$
\left\langle S Q^{2}\left(x_{1}^{p} x_{2}^{q}\right), \frac{x_{1}^{u}}{u !} \frac{x_{2}^{v}}{v !}\right\rangle=\left\langle x_{1}^{p} x_{2}^{q}, S Q_{2}\left(\frac{x_{1}^{u}}{u !} \frac{x_{2}^{v}}{v !}\right)\right\rangle
$$

for all $p, q, u, v$ shows that the differential formula for $S Q_{2}$ is indeed the Kronecker dual of $S Q^{2}$.

From a topologist's point of view, the divided polynomial algebra $\mathbf{W}^{*}(n) \otimes \mathbf{F}_{2}$ is the homology of the product of $n$ copies of real infinite dimensional projective space, on which the Steenrod algebra acts by Kronecker duality. This action is exploited, for example, in studying the splitting of classifying spaces of general linear groups over finite fields [216], and work on the hit problem $[\mathbf{1 5}, \mathbf{1 6}, \mathbf{5 0}]$.

An observation which is sometimes useful in dealing with the action of the Steenrod algebra on $\mathbf{W} \otimes \mathbf{F}_{2}$ is the commutativity with partial differentiation.

LEMMA 2.21.

$$
S q^{n} \frac{\partial}{\partial x_{i}}=\frac{\partial}{\partial x_{i}} S q^{n}
$$

This result is not true for integral Steenrod squares. The proof in the modulo 2 case is a straightforward application of the Cartan formula. A simple application is a quick demonstration that if an element $E$ in $\mathscr{A}$ has excess $k$, then $E\left(x_{1} \cdots x_{m}\right) \neq 0$ for all $m \geqslant k$. 
2.4 Other sources of integral Steenrod squares. In the theory of deformations of Hopf algebras, Duflot [63] introduces an integral version of the Steenrod algebra, starting with the free associative algebra

$$
V=\mathbf{Z}\left[X_{1}, X_{2}, \ldots X_{n}, \ldots\right]
$$

on a countable number of generators $X_{i}$ with $X_{0}=1$. Then $V$ is a Hopf algebra with coproduct determined on generators by

$$
\psi\left(X_{n}\right)=\sum_{0 \leqslant i \leqslant n} X_{i} \otimes X_{n-i} .
$$

Let $V\left[\left[h_{1}, h_{2}\right]\right]$ denote the ring of formal power series over $V$ in the commuting indeterminates $h_{1}, h_{2}$, and let

$$
f(t)=\sum_{i \geqslant 0} t^{i} X_{i}
$$

be the formal power series in the indeterminate $t$. Now introduce

$$
\alpha=h_{1}^{2}+h_{1} h_{2}, \quad \beta=h_{2}^{2}, \quad \delta=h_{2}^{2}+h_{1} h_{2}, \quad \gamma=h_{1}^{2},
$$

and define the ideal $J$ in $V$ in terms of generators obtained by equating coefficients of all monomials $h^{i} h^{j}$ in the identity

$$
f(\alpha) f(\beta)=f(\delta) f(\gamma) .
$$

These relations are designed to fit the Bullett-Macdonald formulae in Theorem 1.15. It is shown in [63] that $J$ is a Hopf ideal, and the quotient $A_{2}(\mathbf{Z})=V / J$ qualifies as an integral version of the Steenrod algebra because its modulo 2 reduction is $\mathscr{A}$. A number of relations are worked out in [63] for the algebra $A_{2}(\mathbf{Z})$. In particular,

$$
X_{1}^{i}=i ! X_{i}, \quad \text { and } \quad X_{i} X_{j}=\left(\begin{array}{c}
i+j \\
j
\end{array}\right) X_{i+j} \quad \bmod C,
$$

where $C$ is the commutator ideal. Furthermore, $C$ consists of the torsion elements in $A_{2}(\mathbf{Z})$. There is at least a superficial resemblance to differential operators if $X_{i}$ is matched with $S Q^{i}$. It should be noted, however, that there is no torsion in the integral Steenrod algebra $\mathscr{N}$ introduced earlier.

Yet another version of the Steenrod algebra comes from the work of Barratt and Miller [20] on conjugation in $\mathscr{A}$, and from similar work of Crossley and Hubbuck [53], relating to $K$-theory operations. Again, using notation slightly modified from that of the authors, Crossley and Hubbuck define an algebra $A(2)$ as a quotient of the free associative algebra $V \otimes \mathbf{F}_{2}=\mathbf{F}_{2}\left[X_{0}, X_{1}, \ldots\right]$. Let $v_{2}(k)$ denote the highest exponent of a power of 2 in $k$. Elements $q_{n}$ are defined recursively by

$$
q_{0}=X_{0}, \quad \sum_{0 \leqslant i \leqslant n} X_{i} q_{n-i}=0
$$

and then elements $p_{n, N}$ are defined for $n>0$ and $N \geqslant n+1+v_{2}(n !)$ by

$$
p_{n, N}=\sum_{0 \leqslant i \leqslant N-n}\left(\begin{array}{c}
n+i \\
i
\end{array}\right) X_{n+i} q_{N-n-i}
$$

The algebra $A(2)$ is the quotient of $V \otimes \mathbf{F}_{2}$ by the ideal generated by the $p_{n, N}$. It is shown in [53] that $A(2)$ is a Hopf algebra with the usual coproduct as described already on generators $X_{n}$ in the Duflot algebra. The map which associates $X_{i}$ to $S q^{i}$ 
induces a surjective Hopf algebra map of $A(2)$ onto $\mathscr{A}$ which fails to be injective for the first time in grading 5 .

2.5 Remarks. It would be interesting to relate the algebras mentioned above and various ways of setting up the Steenrod algebra in Section 1 to differential operators. This programme may involve extending the scope of the differential operators $D_{k}$ to negative $k$ acting on Laurent polynomials in the variables $x_{i}[\mathbf{6}, \mathbf{2 1 5}$.

For interpretations of Landweber-Novikov operations as differential operators in the context of conformal field theory, quantum groups and diffeomorphisms of the line, we refer to $[33,34,35,98,150]$.

It would also be interesting to know if the complex bordism of a compact Hausdorff topological space $X$ could be defined in an algebraic manner, perhaps in terms of the algebra of complex-valued functions on $X$ in the style of algebraic $K$-theory, in such a way as to admit the differential operator algebra as natural operations in a calculus setting.

\subsection{Problems.}

Problem 2.22. Describe an additive basis of the integral Steenrod algebra $\mathscr{N}$ in terms of composites of integral Steenrod squares $S Q^{k}$.

Problem 2.23. Describe relations in the $S Q^{k}$ covering the Adem relations in $\mathscr{A}$.

Problem 2.24. What is the relationship between the integral Steenrod algebra $\mathscr{N}$ arising out of differential operators, Duflot's algebra $A_{2}(\mathbf{Z})$ arising out of Hopf algebra deformation theory, and the Crossley-Hubbuck algebra $A(2)$ ?

Problem 2.25. How are the local formulae for compositions of wedge products of differential operators $D(K)$ obtained from the global exponential form and the convolution product?

Problem 2.26. Can the 'calculus' approach to Landweber-Novikov operations and the Steenrod algebra, in terms of differential operators, be extended to other cohomology theories?

Problem 2.27. Is there any natural interpretation of May's universal Steenrod algebra, the lambda algebra and the Dyer-Lashof algebra in terms of differential operators $D_{k}$, perhaps extended to negative $k$ acting on Laurent series?

Problem 2.28. How can the complex bordism of a topological space be defined in a way that allows a natural action of the differential operator algebra?

\section{Symmetric functions and differential operators}

The basic properties of the ring $\Lambda$ of symmetric functions in a countable number of variables $x_{i}$ are set out in Chapter 1 of Macdonald's book [121]. A symmetric function is a formally infinite sum of monomials in $\mathbf{W}$ which restricts to a symmetric polynomial when, for each $n \geqslant 1$, all variables are put to zero except the first $n$. 
We shall denote by $\Lambda(n)$ the ring of symmetric polynomials in $x_{1}, \ldots, x_{n}$ over the integers.

3.1 The algebraic Thom map. The elementary symmetric functions $e_{i}$ and the power sums $p_{i}$ are defined in the usual ways. Multiplication in $\Lambda$ is defined as for polynomials, and we shall sometimes emphasise it by the dot product. For example,

$$
e_{1}^{2}=e_{1} \bullet e_{1}=\left(x_{1}+x_{2}+\cdots\right)^{2}=2 e_{2}+p_{2} .
$$

For each multiset $K=k_{1}^{r_{1}} k_{2}^{r_{2}} \cdots k_{a}^{r_{a}}$, there is an associated monomial symmetric function $m(K)$ defined to be the smallest symmetric function containing

$$
\left(x_{1} \cdots x_{r_{1}}\right)^{k_{1}}\left(x_{r_{1}+1} \cdots x_{r_{1}+r_{2}}\right)^{k_{2}} \cdots\left(x_{r_{1}+\cdots+r_{a-1}+1} \cdots x_{r_{1}+\cdots+r_{a}}\right)^{k_{a}} .
$$

For example, $m\left(1^{r}\right)=e_{r}$ and $m(k)=p_{k}$. The sum of all the $m(K)$ in a given degree $n$, which is the sum of all monomials in that degree, is called the complete symmetric function $h_{n}$. The usual notation in the literature for the monomial symmetric function is $m_{\lambda}$, where $\lambda$ is a partition.

Note. We shall continue to work with multisets rather than partitions, which are more traditional in symmetric function theory [121], even though at first sight the notation $m(K)$ for the monomial symmetric function is not in keeping with the usage of the previous section on differential operators. There are several reasons for this choice. Given a partition $\lambda=\left(\lambda_{1}, \ldots, \lambda_{n}\right)$ of the number $|\lambda|=\lambda_{1}+\cdots+\lambda_{n}$, and a collection of 'type' $f$ symmetric functions, the notation $f_{\lambda}$ is frequently used to denote the product $f_{\lambda_{1}} f_{\lambda_{2}} \cdots f_{\lambda_{n}}$ of members of the $f$-collection. For example, the family $e_{\lambda}$, as $\lambda$ ranges over all partitions, is an additive basis of $\Lambda$ because $\Lambda$ is the polynomial algebra generated by the elementary symmetric functions. A similar statement is true for the family $h_{\lambda}$. The family $m(K)$, as $K$ ranges over multisets, is also an additive basis of $\Lambda$. The traditional notation $m_{\lambda}$ does not refer to a product in this case. The main reason, however, for the multiset notation is to do with the algebraic Thom map [221]. Let $\pi=x_{1} x_{2} \cdots$ denote the formal product of the variables $x_{i}$. Then the algebraic Thom map $\phi: \mathscr{D} \rightarrow \Lambda$ is given on differential operators by

$$
\phi(D(K))=\frac{D(K)(\pi)}{\pi},
$$

where $D(K)$ is first evaluated on $\pi$ and the result divided by $\pi$. It is shown in [221] that the map $\phi$ sets up an additive isomorphism of $\mathscr{D}$ with $\Lambda$. There are some familiar correspondences of families of functions under the algebraic Thom map.

\section{EXAMPLE 3.1.}

$$
\phi(D(K))=m(K), \quad \phi\left(D_{k}\right)=p_{k}, \quad \phi\left(S Q^{r}\right)=e_{r}, \quad \phi\left(\frac{D_{1}^{\circ r}}{r !}\right)=h_{r} .
$$

We recall that the $D_{k}$ are the primitives in the Hopf algebra $\mathscr{D}$. In $\Lambda$ there is a coproduct $[\mathbf{7 1}, \mathbf{1 2 1}, \mathbf{2 0 8}]$ which makes $\Lambda$ into a Hopf algebra with respect to the dot product. The primitives are the $p_{k}$. We noted that $\phi\left(D_{k}\right)=p_{k}$, and it can be checked that the algebraic Thom map is a coalgebra map. Hence the dot product lifts back to make yet a third Hopf algebra structure on $\mathscr{D}$ with the same coproduct. 
For example,

$$
D_{1} \bullet D_{1}=D_{1} \vee D_{1}+D_{2}
$$

3.2 Products and conjugation in the symmetric algebra. The identities exhibited in Example 3.1 are fairly natural, and it is a matter of taste whether to work in the differential operator algebra or the symmetric function algebra. For example, if we work in the symmetric function algebra, then the Steenrod algebra is generated by the elementary symmetric functions under the composition product. This particular product does not, however, feature prominently in standard treatments of symmetric functions. On the other hand, if we work in the differential operator algebra, then the dot product is somewhat alien. The wedge product is not commonly considered in either model, but note the factorisation

$$
m(K)=m\left(k_{1}\right)^{\vee r_{1}} \vee m\left(k_{2}\right)^{\vee r_{2}} \vee \cdots \vee m\left(k_{a}\right)^{\vee r_{a}},
$$

which is analogous to the factorisation of $e_{\lambda}$ into elementary symmetric functions with respect to the dot product.

There is a problem, therefore, of understanding the relationship between the three products $\vee, \bullet, \circ$. This problem has been treated in a topological context in terms of Chern classes, Stiefel-Whitney classes, cohomology of classifying spaces and Thom complexes $[19,190]$. However, it would be interesting to codify the material in terms of differential operators. The three conjugations $\chi=\chi^{\vee}, \chi^{\bullet}, \chi^{\circ}$ all have the same value $\chi\left(D_{k}\right)=-D_{k}$ on the primitives. If we work in the symmetric algebra, then the conjugation $\chi^{\bullet}$ is, up to sign, Macdonald's involution $\omega$ [121]. For example, the elementary symmetric functions and the complete symmetric functions are related by conjugation: $\chi^{\bullet}\left(e_{j}\right)=(-1)^{j} h_{j}=(-1)^{j} \omega\left(e_{j}\right)$.

Furthermore, there is a product formula for composition of dot products analogous to the product formula in Theorem 2.7 for composites of wedge products. With a similar notation, consider set functions

$$
\Phi:\left\{k_{1}, k_{2}, \ldots, k_{a}\right\} \rightarrow\left\{l_{0}, l_{1}, l_{2}, \ldots, l_{b}\right\}
$$

and define, as in Theorem 2.7, the numerical functions $\tau\left(l_{j}\right)=\#\left\{\Phi^{-1} l_{j}\right\}$ for the number of elements in the inverse image of $l_{j}$, and $\sigma\left(l_{j}\right)=\sum_{k_{i} \in \Phi^{-1} l_{j}} k_{i}$ for the sum of the elements in the inverse image of $l_{j}$. But instead of the falling factorial function $\rho(\Phi)$, define the function $\pi(\Phi)=l_{1}^{\tau\left(l_{1}\right)} l_{2}^{\tau\left(l_{2}\right)} \cdots l_{b}^{\tau\left(l_{b}\right)}$. The following product formula is due to Grant Walker.

THEOREM 3.2 (Walker [208]).

$$
\begin{gathered}
\left(D_{k_{1}} \bullet D_{k_{2}} \bullet \cdots \bullet D_{k_{a}}\right) \circ\left(D_{l_{1}} \bullet D_{l_{2}} \bullet \cdots \bullet D_{l_{b}}\right) \\
=\sum_{\Phi} \pi(\Phi) D^{\bullet}\left(\Phi^{-1}\left(l_{0}\right)\right) \bullet D_{l_{1}+\sigma\left(l_{1}\right)} \bullet D_{l_{2}+\sigma\left(l_{2}\right)} \bullet \cdots \bullet D_{l_{b}+\sigma\left(l_{b}\right)},
\end{gathered}
$$

where, for a multiset $K=k_{1}^{r_{1}} k_{2}^{r_{2}} \cdots k_{a}^{r_{a}}$,

$$
D^{\bullet}(K)=D_{k_{1}}^{\bullet r_{1}} \bullet D_{k_{2}}^{\bullet r_{2}} \bullet \cdots \bullet D_{k_{a}}^{\bullet r_{a}} \text {. }
$$

It should be possible to give global structure equations for the dot product and dot conjugation by analogy with Theorem 2.17 .

A recurrent problem in topology has been the efficient evaluation of the conjugates of compositions of Steenrod squares. Working in the symmetric algebra, the 
following example shows the values of the composition conjugates of the first few elementary symmetric functions (alias the integral Steenrod squares).

EXAMPLE 3.3 .

$$
\begin{aligned}
-\chi^{\circ}\left(e_{1}\right)= & m(1), \\
\chi^{\circ}\left(e_{2}\right)= & m\left(1^{2}\right)+2 m(2), \\
-\chi^{\circ}\left(e_{3}\right)= & m\left(1^{3}\right)+2 m\left(2^{1} 1^{1}\right)+5 m(3), \\
\chi^{\circ}\left(e_{4}\right)= & m\left(1^{4}\right)+4 m\left(2^{2}\right)+5 m\left(3^{1} 1^{1}\right)+2 m\left(2^{1} 1^{2}\right)+14 m(4), \\
-\chi^{\circ}\left(e_{5}\right)= & m\left(1^{5}\right)+2 m\left(2^{1} 1^{3}\right)+4 m\left(2^{2} 1^{1}\right)+5 m\left(3^{1} 1^{2}\right)+10 m\left(3^{1} 2^{1}\right) \\
& +14 m\left(4^{1} 1^{1}\right)+42 m\left(5^{1}\right) .
\end{aligned}
$$

The coefficients of the last terms $m(k)$ are the ubiquitous Catalan numbers, up to sign. In fact, all the coefficients can be expressed as products of Catalan numbers. The argument is an application of the global formula in Theorem 2.17 for conjugation, restricted to the special case of the formal sequence $\mathbf{t}=(t, 0,0, \ldots)$.

EXAMPLE 3.4. The formal sequence $\mathbf{t}=(t, 0,0, \ldots)$ is associated with the polynomial $x+t x^{2}$ whose functional inverse is

$$
\frac{-1+(1+4 t x)^{1 / 2}}{2 t}=x-t x^{2}+2 t^{2} x^{2}-5 t^{3} x^{4}+14 t^{4} x^{5}-\cdots
$$

Apart from sign, this is a generating function for Catalan numbers. The associated formal sequence is

$$
\hat{\mathbf{t}}=\left(-t, 2 t^{2},-5 t^{3}, 14 t^{4}, \ldots,(-1)^{n} c_{n} t^{n}, \ldots\right),
$$

where $c_{n}=\left(\begin{array}{c}2 n \\ n\end{array}\right) /(n+1)$. Now $\exp _{\vee}\left(t D_{1}\right)=1+t S Q^{1}+t^{2} S Q^{2}+\cdots$, and by Theorem 2.17

$$
\begin{aligned}
\chi^{\circ}\left(\exp _{\vee}\left(t D_{1}\right)\right) & =1+t \chi^{\circ}\left(S Q^{1}\right)+t^{2} \chi^{\circ}\left(S Q^{2}\right)+\cdots \\
& =\exp _{\vee}(\langle\hat{\mathbf{t}}, \mathbf{D}\rangle) \\
& =\exp _{\vee}\left(-t c_{1} D_{1}+t^{2} c_{2} D_{2}+\cdots+(-1)^{n} c_{n} t^{n}+\cdots\right) \\
& =\exp _{\vee}\left(-t c_{1} D_{1}\right) \vee \exp _{\vee}\left(t^{2} c_{2} D_{2}\right) \vee \cdots \\
& =\sum_{i \geqslant 0}\left(c_{1}^{i}(-t)^{i} D\left(1^{i}\right)\right) \vee \cdots \vee \sum_{i \geqslant 0}\left(c_{k}^{i}(-t)^{k i} D\left(k^{i}\right)\right) \vee \cdots
\end{aligned}
$$

Comparing coefficients of $t^{n}$ on both sides of this equation, we obtain

$$
(-1)^{n} \chi^{\circ}\left(S Q^{n}\right)=\sum_{i_{1}+2 i_{2}+\cdots+k i_{k}+\cdots=n} c_{1}^{i_{1}} c_{2}^{i_{2}} \cdots D\left(1^{i_{1}} 2^{i_{2}} \cdots\right) .
$$

Now $c_{r}$ is an even number unless $r=2^{\lambda}-1$. Hence, reducing modulo 2, we obtain Milnor's formula in the Steenrod algebra, which states that the conjugate of a squaring operation is the sum of the Milnor basis elements in the same degree:

$$
\chi^{\circ}\left(S q^{n}\right)=\sum_{j_{1}+3 j_{2}+\cdots+\left(2^{\lambda}-1\right) j_{\lambda}+\cdots=n} D\left(1^{j_{1}} 3^{j_{2}} \cdots\left(2^{\lambda}-1\right)^{j_{\lambda} \cdots}\right) .
$$

Conjugation formulae for elements in $\mathscr{D}$ can be found in [208]. 
EXAMPLE 3.5 .

$$
\begin{aligned}
-\chi^{\circ}\left(D_{k} \vee D_{l} \vee D_{m}\right)= & D_{k} \vee D_{l} \vee D_{m}+(k+l+2) D_{k+l} \vee D_{m} \\
& +(k+m+2) D_{k+m} \vee D_{l}+(l+m+2) D_{l+m} \vee D_{k} \\
& +(k+l+m+3)(k+l+m+2) D_{k+l+m}
\end{aligned}
$$

It would be interesting to compare such formulae with Li's version of Milnor's formulae [116] for conjugation in the Steenrod algebra.

3.3 Remarks. Other bases of the symmetric function algebra, not yet mentioned, are provided by the forgotten functions and the Schur functions. The latter play an important part in the representation theory of the symmetric group [121, 206] and the cohomology of Grassmannians. The Schur basis is related to standard bases by transition matrices of Kostka numbers. It would be interesting to investigate how much of the material in [121] on symmetric functions translates naturally into the language of differential operators, and to see if any light can be cast on the standard combinatorics of Young tableaux by the use of differential operators and Steenrod squares.

Since the differential operators $D(K)$ are symmetric in the variables and derivatives, the action of $\mathscr{D}$ on $\mathbf{W}$ preserves the algebra $\Lambda(n)$ of symmetric polynomials in $n$ variables, and this raises the question of finding closed formulae in $\Lambda(n)$ for the action of $D(K)$ on prescribed symmetric polynomials.

From a topologist's point of view, $\Lambda(n)$ (with suitable conventions about grading) is identified with the cohomology over the integers of the classifying space $B U(n)$ of the unitary group $U(n)$, and the elementary symmetric functions are called 'universal Chern classes' in this context. The classifying space $B U(n)$ is so called because a complex $n$-plane bundle $E$ over a connected compact Hausdorff base space $X$ is induced from a certain universal bundle over $B U(n)$ by a map of $X$ into $B U(n)$. The Thom isomorphism $[\mathbf{1 3 4}, \mathbf{1 9 9}]$

$$
\phi: \mathbf{H}^{*}(X) \rightarrow \tilde{\mathbf{H}}^{*+n}\left(E^{*}\right)
$$

is an isomorphism from the cohomology of $X$ to the reduced cohomology of the one-point compactification $E^{*}$ of $E$, known as the Thom complex of $E$. For the universal bundle $B U(n)$, this Thom complex is denoted by $M U(n)$, and its integral cohomology is identified with the elements of $\Lambda(n)$ divisible by $\pi_{n}=$ $x_{1} \cdots x_{n}$. The Thom isomorphism in this case is identified with multiplication by $\pi_{n}$. Similar remarks apply to real vector bundles, the classifying space $B O(n)$ of the real orthogonal group $O(n)$, and the Thom isomorphism for cohomology modulo 2 . The universal Stiefel-Whitney classes $w_{m}$ are the modulo 2 reductions of the elementary symmetric function $e_{m}$, and generate the modulo 2 cohomology of the classifying space $B O(n)$. For a real vector bundle $E$ over $X$, the Stiefel-Whitney classes $w_{m}$ of the bundle are elements in the cohomology of $X$. They are related to Steenrod squares acting on the cohomology of the Thom complex $E^{*}$ by the formula

$$
w_{m}=\phi^{-1} S q^{m} \phi(1),
$$

where $1 \in \mathbf{H}^{0}(X)$ is the identity element. In the universal case, the formula is the composite of the action of the Steenrod square on $\pi_{n}$ and division by $\pi_{n}$. This offers some explanation for the use of the term 'algebraic Thom map' linking the 
differential operator algebra to the symmetric function algebra introduced at the beginning of the section.

As well as the interpretation of the Steenrod squares in terms of elementary symmetric functions via the algebraic Thom map, the Steenrod algebra also acts on $\Lambda(n) \otimes \mathbf{F}_{2}$, as we explained in a purely algebraic way by the symmetry of differential operators, or topologically by the identification of $\Lambda(n) \otimes \mathbf{F}_{2}$ with $\mathbf{H}^{*}\left(B O(n) ; \mathbf{F}_{2}\right)$. The Steenrod algebra also acts on the cohomology of the Thom complex $\mathbf{H}^{*}\left(M O(n) ; \mathbf{F}_{2}\right)$ viewed as the symmetric polynomials divisible by $x_{1} \cdots x_{n}$. Closed formulae for these actions have been of interest to algebraic and differential topologists, for example in the immersion theory of manifolds $[64,65,66,68]$.

Classically, the Wu formulae $[\mathbf{1 3 4}, \mathbf{2 2 3}]$ answer the question of how the Steenrod squares act on the elementary symmetric polynomials in $\Lambda(n) \otimes \mathbf{F}_{2}$ :

$$
S q^{k}\left(w_{m}\right)=w_{k} w_{m}+\left(\begin{array}{c}
k-m \\
1
\end{array}\right) w_{k-1} w_{m+1}+\cdots+\left(\begin{array}{c}
k-m \\
k
\end{array}\right) w_{m+k},
$$

where the negative binomial coefficients are reduced modulo 2 .

Work on Wu-type formulae appears in $[4,28,30,31,82,110,157,172,192]$. Recently, Lenart [114] has produced integral Wu formulae using differential operators in conjunction with Schur functions, Schubert varieties and the Hammond operators of classical combinatorics.

\subsection{Problems.}

Problem 3.6. How are Schur functions and the forgotten functions related to integral Steenrod squares and differential operators?

Problem 3.7. Can the differential operator approach to the symmetric function algebra throw any light on Kostka numbers and other numbers arising out of transition matrices between various bases of $\Lambda$ ?

Problem 3.8. Explore the interrelations between the dot product, wedge product and composition product in the divided differential operator algebra.

Problem 3.9. Find combinatorial interpretations for the coefficients which occur in the expansions of the various conjugations in the differential operator algebra.

Problem 3.10. What is the significance of the order of a differential operator when translated into the symmetric function algebra under the algebraic Thom map?

\section{Bases, excess and conjugates}

For a variety of reasons, the Milnor basis is the most popular choice when it comes to studying problems in the Steenrod algebra, especially those to do with products, conjugation and excess. Perhaps the interpretation of Milnor basis elements in terms of differential operators offers some explanation for their significance. For example, the notion of excess has a natural meaning in terms of differential operators. Kraines shows in [103] that the excess of Milnor's basis element $S q\left(r_{1}, r_{2}, \ldots, r_{a}\right)$ is

$$
r_{1}+r_{2}+\cdots+r_{a}
$$


and by Lemma 2.2, we can now interpret excess in the following simple way.

Lemma 4.1. The excess of the element

$$
E=\frac{D_{1}^{\vee r_{1}}}{r_{1} !} \vee \frac{D_{3}^{\vee r_{2}}}{r_{2} !} \vee \cdots \vee \frac{D_{2^{a}-1}^{\vee r_{a}}}{r_{a} !}
$$

is the order of $E$, in the usual sense for differential operators.

This conforms to the original definition of excess, because the differential operator $D(K)$, associated with the multiset $K=k_{1}^{r_{1}} k_{2}^{r_{2}} \cdots k_{a}^{r_{a}}$, has order $e=r_{1}+r_{2}+\cdots+r_{a}$, annihilates all polynomials of degree less than $e$, and is non-zero on the product of $e$ variables.

The term of highest order in the composition of two differential operators $D(K)$ and $D(L)$ is the wedge product $D(K) \vee D(L)$. In the Steenrod algebra this corresponds to the following statement, which can be found in Chapter 15 of [125], namely

$$
S q\left(r_{1}, r_{2}, \ldots, r_{a}\right) S q\left(s_{1}, s_{2}, \ldots, s_{b}\right)=\varepsilon S q\left(r_{1}+s_{1}, r_{2}+s_{2}, \ldots\right)+E,
$$

where $\varepsilon$ is 0 or 1 , and the error term $E$ has excess strictly lower than that of $S q\left(r_{1}+s_{1}, r_{2}+s_{2}, \ldots\right)$. Since $\varepsilon$ may be zero in the modular case, the task of finding the highest-order terms in a product of elements in the Steenrod algebra is not so straightforward. The same applies to the problem of finding lowest-order terms and excess of iterated composites.

One major reason for the importance of the Milnor basis is the algorithmic product formula in Theorem 1.7, which no other basis seems to possess in such a workable form. As we saw in Section 2, even at the integral level, there are combinatorial formulae for evaluating compositions and conjugates. Another reason for the importance of the Milnor basis is the fact that a Hopf subalgebra of $\mathscr{A}$ is generated as a vector space by the Milnor basis elements which lie in it [125]. For example, the algebra $\mathscr{A}(n)$ is a Poincaré algebra with top element

$$
S q\left(2^{n+1}-1,2^{n}-1, \ldots, 3,1\right),
$$

and a basis for $\mathscr{A}(n)$ consists of the elements $S q\left(r_{1}, \ldots, r_{n+1}\right)$ with $r_{i}<2^{n+2-i}$. The admissible basis is not so convenient. For example, the element

$$
S q^{5}+S q^{4} S q^{1}
$$

which is the sum of two admissible monomials each lying in $\mathscr{A}(2)$, actually lies in $\mathscr{A}(1)$, as we see from the Adem relation

$$
S q^{2} S q^{3}=S q^{5}+S q^{4} S q^{1}
$$

A summary of the various bases now available in the Steenrod algebra can be found in Monks' paper [143], together with information about the transition matrices between them. We shall just make a few comments on certain particular features of some of these bases.

4.1 The admissible basis. Ken Monks [143] has investigated the question of when certain bases of the Steenrod algebra are triangularly related to the Milnor basis with respect to suitable orderings. The admissible basis has this property with respect to the right lexicographic ordering. 
Consider the Milnor element $S q\left(r_{1}, r_{2}, \ldots, r_{a}\right)$, and define $t_{a}=r_{a}$ and $t_{i}=r_{i}+2 t_{i+1}$ for $1 \leqslant i<a$. The excess of $S q^{t_{1}} S q^{t_{2}} \cdots S q^{t_{a}}$ is

$$
2 t_{1}-\left(t_{1}+t_{2}+\cdots+t_{a}\right)=r_{1}+r_{2}+\cdots+r_{a}=e .
$$

In Lemma 8 of Milnor's paper $[\mathbf{1 3 1}]$ and in $[\mathbf{1 4 3}, \mathbf{2 1 0}]$, we find the following result.

LEMMA 4.2.

$$
S q\left(r_{1}, r_{2}, \ldots, r_{a}\right)=S q^{t_{1}} S q^{t_{2}} \cdots S q^{t_{a}}+E,
$$

where the error term $E$ has excess strictly greater than $e$.

Monks' condition for the error term $E$ to be zero is proved in [210], and can be explained in terms of the function $2^{\omega(r)}$ which designates the smallest power of 2 not less than $r$.

TheOREM 4.3 (Monks $[\mathbf{1 4 3}, \mathbf{2 1 0}]$ ). The Milnor basis element $S q\left(r_{1}, r_{2}, \ldots, r_{m}\right)$ is also an admissible basis element, and is therefore equal to $S q^{t_{1}} S q^{t_{2}} \cdots S q^{t_{a}}$, if and only if $r_{i} \equiv-1 \bmod 2^{\omega\left(r_{i+1}\right)}$ for all $1 \leqslant i<m$.

For example, the top element of $\mathscr{A}(2)$ is $S q^{17} S q^{5} S q^{1}$.

Another numerical function which frequently occurs in the study of the Steenrod algebra is defined as follows.

Definition 4.4. Let $\mu(f)$ denote the smallest number of positive integers of the form $2^{\lambda}-1$ adding up to $f$, and let $\zeta(f)=(f+\mu(f)) / 2$.

Given a number $f$, following Singer [182], we construct an admissible sequence as follows:

$$
\zeta_{1}=\zeta(f), \quad \zeta_{2}=\zeta\left(f-\zeta_{1}\right), \quad \ldots, \quad \zeta_{r}=\zeta\left(f-\zeta_{1}-\cdots-\zeta_{r-1}\right) .
$$

The sequence stops when $\zeta_{r+1}=0$. For example, the sequence for $f=7$ is $4,2,1$, and the sequence for $f=8$ is $5,2,1$.

THEOREM 4.5 (Singer [182]). The largest term, in right lexicographic order, in the expansion of $\chi\left(S q^{f}\right)$ in the admissible basis is $S q^{\zeta_{1}} S q^{\zeta_{2}} \cdots S q^{\zeta_{r}}$.

As it happens, $S q^{\zeta_{1}} S q^{\zeta_{2}} \cdots S q^{\zeta_{r}}$ is the largest term in right lexicographic order among all admissibles in grading $f$, and is also the term of least excess. Recently, the following result has been established by Judith Silverman.

TheOrem 4.6 (Silverman [178]). The excess of $\chi\left(S q^{2^{k-1} f} S q^{2^{k-2} f} \cdots S q^{2 f} S q^{f}\right)$ is $\left(2^{k}-1\right) \mu(f)$.

One might conjecture that the largest term in right lexicographic order in the admissible expansion of $\chi\left(S q^{2^{k-1} f} S q^{2^{k-2} f} \cdots S q^{2 f} S q^{f}\right)$ is

$$
S q^{\left(2^{k}-1\right) \zeta_{1}} S q^{\left(2^{k}-1\right) \zeta_{2}} \cdots S q^{\left(2^{k}-1\right) \zeta_{r}} .
$$

This element is not, in general, the largest element over all elements of the same grading. 
4.2 The $P_{t}^{s}$ bases. We call a number of the form $2^{s}\left(2^{t}-1\right)$ atomic. Most of the bases of recent origin are assembled from strings of atomic squares $S q^{2^{s}\left(2^{t}-1\right)}$ in various orders, or from Milnor elements $P_{t}^{s}$ in atomic degrees $2^{s}\left(2^{t}-1\right)$. It is convenient to arrange the $P_{t}^{s}$ family as in Margolis' table in Chapter 15 of [125].

EXAMPLE 4.7.

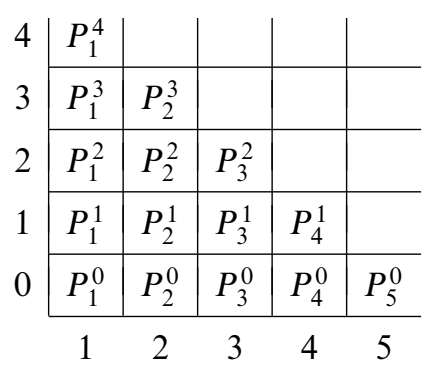

The element $P_{t}^{s}$ is placed in position $(t, s)$ in cartesian coordinates. The relationship between the following theorem of Monks and differential operators can be found in [221].

Theorem 4.8 (Monks [143]). Strings of distinct $P_{t}^{s}$, taken in a fixed but arbitrary order under composition, form a basis of the Steenrod algebra, and this basis is triangularly related to the Milnor basis.

The $P_{t}^{s}$ family is particularly useful in handling questions about Hopf subalgebras of $\mathscr{A}$. Information relating Hopf subalgebras of the Steenrod algebra to shapes in the $(s, t)$-plane defined by profile functions can be found in Margolis [125].

ExAmple 4.9. The top element of $\mathscr{A}(n)$ is the product of the elements $P_{t}^{s}$, in any order, satisfying $s+t \leqslant n+1$. A basis for $\mathscr{A}(n)$ is obtained by taking subproducts in the given order. The situation may be described geometrically by the triangle $s+t \leqslant n+1$ in the $(s, t)$-plane. The elements $P_{1}^{s}=S q^{2^{s}}$ along the vertical side of the triangle, $s \leqslant n$, form a minimal algebraic generating set of $\mathscr{A}(n)$. The elements along the base of the triangle, $P_{t}^{0}=S q(0, \ldots, 0,1,0, \ldots)$, form an exterior subalgebra of $\mathscr{A}(n)$. Furthermore, $\left(P_{t}^{s}\right)^{2}=0$ if and only if $s<t$, and in this case the elements are fixed by conjugation, $\chi\left(P_{t}^{s}\right)=P_{t}^{s}$.

To explain some of these formulae in the language of differential operators, recall that the primitive elements $D_{2^{t}-1}$, corresponding to $P_{t}^{0}$, satisfy the Lie bracket formulae

$$
\left[D_{2^{t_{1}}-1}, D_{2^{t_{2}-1}}\right]=\left(2^{t_{2}}-2^{t_{1}}\right) D_{2^{t_{2}}+2^{t_{1}}-2},
$$

and therefore commute when reduced modulo 2. Furthermore, the composition product formula in Theorem 2.7 shows in general that $D_{k} \circ D_{l}=D_{k} \vee D_{l}+(l+1) D_{k+l}$, and in particular that

$$
\left(D_{2^{t}-1}\right)^{\circ 2}=\left(D_{2^{t}-1}\right)^{\mathrm{V} 2}+2^{t} D_{2^{t+1}-2} .
$$

Now $\left(D_{2^{t}-1}\right)^{\vee 2}$ is divisible by 2 . This explains the formula $\left(P_{t}^{0}\right)^{2}=0$ in the Steenrod algebra. The more general formula $\left(P_{t}^{s}\right)^{2}=0$, for $s<t$, follows from the modulo 2 reduction of Example 2.10. 
4.3 Arnon's C-basis. The question arises of whether it is possible to write down a basis of $\mathscr{A}$ which is monomial in the $S q^{i}$ and at the same time restricts to a basis $\mathscr{A}(n)$ for all $n$ in the way that the Milnor basis does. As we have noted in Problems 1.8, this question was first broached by Wall [213], who constructed a basis in terms of strings of $S q^{2^{k}}$. Wall's basis is not triangularly related to Milnor's basis. Dan Arnon rediscovered Wall's basis and found several others, in particular the C-basis which is triangularly related to Milnor's basis. To describe the C-basis, we call a string of squares

$$
S q^{t_{1}} S q^{t_{2}} \cdots S q^{t_{m}}
$$

$C$-admissible if $t_{i} \leqslant 2 t_{i+1}$ for $1 \leqslant i<m$ and $t_{m-i}$ is divisible by $2^{i}$. Then one of Arnon's results states the following.

THEOREM 4.10 (Arnon [17]). The set of $C$-admissible monomials is a basis of the Steenrod algebra.

For example, the elements

$$
S q^{2^{k-1} f} S q^{2^{k-2} f} \cdots S q^{2 f} S q^{f}, \quad\left(S q^{2^{k}}\right)^{k+1}
$$

belong to the C-basis for all $f, k \geqslant 1$, but $\left(S q^{8}\right)^{5}$ does not. The following result exhibits a nice property of the $\mathrm{C}$-basis elements.

Lemma 4.11. The excess of a C-admissible element is the superfix of its last factor.

The proof is a straightforward induction on the number of factors, observing that

$$
S q^{2 s} S q^{t}\left(x_{1} \cdots x_{t}\right)=\left(S q^{s}\left(x_{1} \cdots x_{t}\right)\right)^{2},
$$

and this is non-zero if $s \leqslant t$, as observed after Lemma 2.21 .

4.4 The $Y$-basis and Z-basis. Yet further families of monomial bases in atomic squares have recently emerged [220], which respect the $\mathscr{A}(n)$, but are not triangularly related to Milnor's basis. We first consider two orderings of atomic numbers, called the $\mathrm{Y}$-order and the $\mathrm{Z}$-order.

The $\mathrm{Y}$-order is given by

$$
1,3,7,15, \ldots, 2,6,14,30, \ldots, 4,12,28,60, \ldots, 8, \ldots
$$

and the $\mathrm{Z}$-order is given by

$$
1,3,2,7,6,4,15,14,12,8, \ldots
$$

In the Y-order, all numbers of the form $2^{r}-1$ are listed first in their natural order, then their doubles, quadruples, etc. The Z-order of the atomic numbers is induced by the ordering of all natural numbers

$$
1,3,2,7,6,5,4,15,14,13,12,11,10,9,8, \ldots
$$

in which intervals of the form $\left[2^{n}, \ldots, 2^{n+1}-1\right]$, in their natural order, have been reversed. 
THEOREM 4.12 (Wood [220]). The top element of $\mathscr{A}(n)$ is the product of the atomic squares $S q^{2^{s}\left(2^{t}-1\right)}$ in reverse $Y$-order with superfix not greater than $2^{n+1}-1$, and the set of substrings of this element is a basis of $\mathscr{A}(n)$. A similar statement applies to the Z-order.

For example, the top element of $\mathscr{A}(3)$ is given by each of the following expressions in the reverse $\mathrm{Y}$-order and Z-order, respectively.

EXAMPLE 4.13.

$$
\begin{aligned}
& S q^{8} S q^{12} S q^{4} S q^{14} S q^{6} S q^{2} S q^{15} S q^{7} S q^{3} S q^{1}, \\
& S q^{8} S q^{12} S q^{14} S q^{15} S q^{4} S q^{6} S q^{7} S q^{2} S q^{3} S q^{1} .
\end{aligned}
$$

A basis for $\mathscr{A}(3)$ consists of the $2^{10}$ strings of atomic squares that can be selected from the top string in the given order. Since these bases are not triangularly related to Milnor's basis, it is a problem to calculate the excess and find closed formulae for products in the given basis. However, one nice feature of the Z-basis is that the bases of the subalgebras $\mathscr{A}(n)$ build up from right to left. The Steenrod algebra is free as a right module over $\mathscr{A}(n)$. This is a useful property for handling problems about right multiplication in the Steenrod algebra. For example, to find the kernel $K$ of right multiplication by $S q^{2^{n}}$ in $\mathscr{A}$, it is enough to find the kernel $K(n)$ of this action in the subalgebra $\mathscr{A}(n)$. A basis for $K$ has the form $E F$, where $E$ is a Z-basis element whose last factor is higher than $S q^{2^{n+1}-1}$ in the Z-order, and $F$ is a basis element for $K(n)$.

ExAmple 4.14. The kernel of right multiplication by $S q^{1}$ in $\mathscr{A}$ is the left ideal generated by $S q^{1}$ and has a basis of the form $E S q^{1}$, where $E$ is a Z-basis element whose last factor is higher than $S q^{1}$.

We refer to the pioneering work of Toda $[\mathbf{1 4 7}, \mathbf{1 4 8}, 201]$ and Wall [213] for information on kernel problems of this kind.

4.5 Wall's relations. We return now to the topic of Wall's relations $[\mathbf{1 5 3}, \mathbf{2 1 3}]$. It will be found convenient to write

$$
\widehat{E}=\chi(E)
$$

for the conjugate of an element $E$ in the Steenrod algebra.

It has been clear since the work of Barratt and Miller [20], Davis [55, 56], Wall [213] and other contributors $[21,49,70,116,191]$ that among the most fruitful relations in the Steenrod algebra are those which express rules for the evaluation of the product $E \widehat{F}$ of an element with a conjugate element, and provide efficient formulae for the evaluation of conjugation itself. For example, the following formulae have been found useful in handling nilpotence questions.

EXAMPLE 4.15. For positive integers $a, b$,

$$
\widehat{S q^{2^{a}}} S q^{2^{a}\left(2^{b}-1\right)}=S q^{2^{a-1}\left(2^{b+1}-1\right)} \widehat{S q^{2^{a-1}}} .
$$


One way to establish these results is through the work of Davis [55], as extended by Silverman [174], which exploits Milnor's product formula. Li [116] has reproduced the results in terms of his global product formulae. Following [174], we say that if $a=\sum_{i \geqslant 0} a_{i} 2^{i}$ and $b=\sum_{i \geqslant 0} b_{i} 2^{i}$ are integers $\geqslant 0$, where $a_{i}, b_{i} \in\{0,1\}$, then $a$ dominates $b$ if $a_{i} \geqslant b_{i}$ for all $i$, and write $a \geq b$. This is equivalent to the condition that the binomial coefficient $\left(\begin{array}{l}a \\ b\end{array}\right)$ is odd. For a sequence $R=\left(r_{1}, \ldots, r_{k}\right)$, we recall that the degree of $S q(R)$ is $|R|=\sum_{i \geqslant 1}\left(2^{i}-1\right) r_{i}$, and the excess of $S q(R)$ is $\sum_{i \geqslant 1} r_{i}$. The Davis-Silverman $[\mathbf{5 5}, \mathbf{1 1 6}, \mathbf{1 7 4}]$ relations are

$$
\begin{gathered}
S q^{u} \widehat{S q^{v}}=\sum_{R} S q(R):|R|=u+v, \quad|R|+\sum_{i \geqslant 1} r_{i} \geq 2 u, \\
\widehat{S q^{u}} S q^{v}=\sum_{R} S q(R):|R|=u+v, \quad \sum_{i \geqslant 1} r_{i} \geq v .
\end{gathered}
$$

Formulae of this kind are also implicit in the work of Barratt and Miller [20], which modifies the formulation of the Adem relations given by Bullett and Macdonald [36]. Among these relations (also established by Walker [207] and by Crabb, Crossley and Hubbuck [49] using $K$-theory methods) are the expressions for the conjugates of the squaring operations.

EXAMPLE 4.16.

$$
\widehat{S q^{k}}=\sum_{u-1 \geq k^{\prime}-1} S q^{u} \widehat{S q^{v}}
$$

where $k^{\prime}=(k+\mu(k)) / 2$ and $\mu(k)$ is as in Definition 4.4. This confirms the earlier statement, in Theorem 4.5, that the excess of $\widehat{S q^{k}}$ is $\mu(k)$. Here are some particular cases:

$$
\begin{gathered}
\widehat{S q^{19}}=S q^{11} \widehat{S q^{8}}+S q^{12} \widehat{S q^{7}}+S q^{15} \widehat{S q^{4}}+S q^{16} \widehat{S q^{3}} \\
\widehat{S q^{55}}=S q^{30} \widehat{S q^{25}}+S q^{32} \widehat{S q^{23}}, \\
\widehat{S q^{58}}=S q^{32} \widehat{S q^{26}} .
\end{gathered}
$$

Further formulae can be found in Ken Monks' article [139]. A special case of interest is Straffin's formula.

$$
\text { THEOREM } 4.17 \text { (Straffin [191]). } \widehat{S q^{2^{n}}}=S q^{2^{n}}+S q^{2^{n-1}} \widehat{S q^{2^{n-1}}} \text {. }
$$

This provides an efficient way of finding the conjugates of an algebraic generating set of the Steenrod algebra, and leads to the idea that conjugation should perhaps be somehow incorporated into the Steenrod algebra as a defining part of its structure. Wall went some way to doing this in [213], where he defined a set of relations for the Steenrod algebra involving the $S q^{2^{k}}$ modulo $\mathscr{A}(k-1)$, as explained in Theorem 1.9. These relations can, in fact, be expressed in closed form if the conjugation operator is brought into play.

THEOREM 4.18 (Walker [207]). Closed forms of the Wall relations are

(i) $S q^{2^{i}} S q^{2^{i}}=S q^{2^{i-1}} \widehat{S q^{2 i}} S q^{2^{i-1}}+S q^{2^{i-1}} S q^{2^{i-1}} \widehat{S q^{2^{i}}}$,

(ii) $S q^{2^{i}} \widehat{S q^{2 j}}+\widehat{S q^{2 j}} S q^{2^{i}}=\widehat{S q^{2^{j+1}}} S q^{2^{i}-2^{j}}$ for $i-j \geqslant 2$.

$A$ third relation may be added:

(iii) $S q^{2^{i-1}} \widehat{S q^{2^{i}}}+\widehat{S q^{2^{i}}} S q^{2^{i-1}}=\widehat{S q^{2^{i-1}}} S q^{2^{i}}+S \widehat{q^{2^{i}+2^{i-1}}}$. 
Wall's original relations can be recovered by removing 'hats', and in principle the 'error' terms of Theorem 1.9 can be written down explicitly in $\mathscr{A}(i-1)$. For example, in relation (i) we have, by Straffin's formula,

$$
\begin{aligned}
S q^{2^{i}} S q^{2^{i}}= & S q^{2^{i-1}}\left(S q^{2^{i}}+S q^{2^{i-1}} \widehat{S q^{2^{i-1}}}\right) S q^{2^{i-1}} \\
& +S q^{2^{i-1}} S q^{2^{i-1}}\left(S q^{2^{i}}+S q^{2^{i-1}} \widehat{S q^{2^{i-1}}}\right) \\
= & S q^{2^{i-1}} S q^{2^{i}} S q^{2^{i-1}}+S q^{2^{i-1}} S q^{2^{i-1}} S q^{2^{i}} \\
& +S q^{2^{i-1}} S q^{2^{i-1}} \widehat{S q^{2^{i-1}}} S q^{2^{i-1}}+S q^{2^{i-1}} S q^{2^{i-1}} S q^{2^{i-1}} \widehat{S q^{2^{i-1}}},
\end{aligned}
$$

which is Wall's formula with the explicit error term in $\mathscr{A}(i-1)$.

\subsection{Problems.}

Problem 4.19. Which orderings of atomic Steenrod squares give rise to bases of the $\mathscr{A}(n)$ ?

Problem 4.20. How is excess computed for the Y-basis and Z-basis?

Problem 4.21. Find efficient formulae for the excess of conjugates of composites of Steenrod squares.

Problem 4.22. Are there any bases of the Steenrod algebra, other than the Milnor basis, which admit a workable product formula?

Problem 4.23. Are there analogues of atomic bases for the integral Steenrod algebra and the odd prime cases?

Problem 4.24. Can the Steenrod algebra be developed from the closed versions of Wall's formulae in a way that incorporates conjugation as a defining part of the structure? Are there integral and modulo $p$ analogues?

Problem 4.25. Develop explicit formulae in the differential operator algebra for products of the form $D(K) \circ \chi(D(L))$.

Problem 4.26. Describe the fixed point set of conjugation $\chi^{\circ}$ in $\mathscr{A}, \mathscr{D}$ and related Hopf algebras [54].

\section{The stripping technique}

In theory, any relation $E=0$ in the Steenrod algebra can be detected by evaluating $E$ on the product of variables $x_{1} \cdots x_{n}$ if the grading of $E$ does not exceed $n$. In practice, however, this is not a feasible method of proceeding, except in certain cases. The combinatorial problem of keeping track of binomial coefficients modulo 2 gets out of hand for long strings of Steenrod squares, as will be discovered by anyone who cares to prove that $\left(S q^{32}\right)^{12}=0$ by this method. It does succeed, however, for short strings. For example, the relation $S q^{2 k-1} S q^{k}=0$ can be demonstrated in this way for all values of $k \geqslant 0$. Similar remarks apply to the difficulties arising from the iterated use of Adem relations, as well as the dual approach using the coproduct 
and the global composition formulae of Li [116]. We mentioned in Theorem 1.12 that all relations in $\mathscr{A}(n)$ are generated from $S q^{2 k-1} S q^{k}=0$, for any $k$ large enough, by the stripping process. It is sometimes possible to short-cut otherwise prohibitively complicated methods for producing relations in the Steenrod algebra by starting with a known relation and then stripping it down to the required form. The technique works in any Hopf algebra, in the sense that the algebra acts on its dual. However, the success of the method depends on how easily the action can be implemented in specified bases of the algebra. By combining the stripping technique and a reverse process, which we call 'strapping', it is shown how all relations in the Steenrod algebra come from the basic relation $S q^{1} S q^{1}=0$.

5.1 Stripping in the Steenrod algebra. We shall now explain in more detail how the stripping process works in the Steenrod algebra. Call a vector allowable if its entries consist of adjacent descending powers of two. For example, (1), $(4,2)$, $(8,4,2,1)$ are allowable, but $(4,1)$ is not. Given a monomial $X=S q^{r_{1}} S q^{r_{2}} \cdots S q^{r_{n}}$ in Steenrod squares with superfix vector $w=\left(r_{1}, r_{2}, \ldots, r_{n}\right)$, and given an allowable vector $v$, first build up $v$ in all possible ways into vectors $v_{1}, v_{2}, \ldots$ of the same dimension, $n$, as $w$ by inserting zeros, if necessary. Then write down the polynomial whose monomial superfixes are the vectors $w-v_{1}, w-v_{2}, \ldots$ We refer to this polynomial as the monomial $X$ stripped by the allowable vector $v$. If the dimension of $v$ already exceeds that of $w$, or an entry of $v_{i}$ exceeds the corresponding entry of $w$, then we count the result of stripping as zero (not to be confused with stripping $(2,1)$ from $S q^{2} S q^{1}$, which gives $\left.S q^{0}\right)$. Clearly, the process extends to a polynomial in the Steenrod algebra, and it can be shown that the process is compatible with the Adem relations. Hence a relation stripped by an allowable vector gives a new relation. The following routine illustrates the process.

EXAMPLE 5.1. Take the relation $S q^{17} S q^{9}=0$, and strip by the vector (16) to obtain $S q^{1} S q^{9}=0$. Also strip by the vector (8), to obtain $S q^{9} S q^{9}+S q^{17} S q^{1}=0$. Next multiply on the right by $S q^{9}$, giving $S q^{9} S q^{9} S q^{9}=0$. Now take the two-dimensional vector $(2,1)$, and consider all the ways of making this vector into a three-dimensional vector by inserting zeros, namely $(0,2,1),(2,0,1),(2,1,0)$. Strip from $(9,9,9)$ to finish with the relation

$$
S q^{9} S q^{7} S q^{8}+S q^{7} S q^{9} S q^{8}+S q^{7} S q^{8} S q^{9}=0 .
$$

The quickest way to demonstrate why stripping works is to consider a variable $x$ and a general polynomial $f$ independent of $x$. Applying a given element $E$ in the Steenrod algebra to $x f$, and using the Cartan formula, produces an expansion of the form

$$
x E(f)+x^{2} \omega_{1}(E)(f)+x^{4} \omega_{(2,1)}(E)(f)+\cdots,
$$

where $\omega_{v}$ denotes the operation of stripping by the vector $v$. If $E=0$ is a relation in the Steenrod algebra, then by picking off the coefficients of $x^{2}, x^{4}, \ldots$, we obtain the relation stripped by the vectors $(1),(2,1), \ldots$, because $f$ is a general polynomial. The same argument, replacing $x$ by $x^{2^{k}}$, shows why stripping works for a general allowable vector. Iterated stripping by $(2,1)$ of the relation $S q^{2^{k}-1} S q^{2^{k-1}}=0$ produces relations $S q^{2 m-1} S q^{m}=0$ in lower grading, and further stripping by the vector (1) produces the Adem relations. 
It is clear from the definition that stripping operators commute. Hence the set of stripping operators $\omega_{v}$, as $v$ ranges over allowable vectors, constitutes a commutative algebra $\Omega$. In fact, $\Omega$ is canonically isomorphic to the dual Steenrod algebra $\mathscr{A}^{*}$ as a polynomial algebra. To set up the isomorphism, we associate $\xi_{n}^{2^{k}}$ in $\mathscr{A}^{*}$ with the stripping operator $\omega_{v}$, where $v=\left(2^{k+n-1}, \ldots, 2^{k}\right)$, and extend to an arbitrary power $\xi_{n}^{r}$ by first expressing $r$ in binary form and then taking the composite of the corresponding stripping operators. The construction is then extended to an arbitrary monomial in the $\xi_{n}$, and finally to $\mathscr{A}^{*}$ by linearity. For example, $\xi_{1}^{5} \xi_{2}^{3}$ corresponds to the stripping operator $\omega_{(1)} \omega_{(4)} \omega_{(2,1)} \omega_{(4,2)}$. Under this isomorphism, it is now straightforward to check that the stripping process is expressed by the composite map mentioned in Section 1.

LeMma 5.2. The stripping process is given by the composite map

$$
\mathscr{A}^{*} \otimes \mathscr{A} \stackrel{1 \otimes \psi}{\longrightarrow} \mathscr{A}^{*} \otimes \mathscr{A} \otimes \mathscr{A} \stackrel{\kappa \otimes 1}{\longrightarrow} \mathscr{A},
$$

where $\psi$ is the coproduct and $\kappa$ is the Kronecker product.

For example, following the map through on the element $\xi_{n} \otimes E$, for $\xi_{n} \in \mathscr{A}^{*}$ and a monomial $E$ in Steenrod squares, we first write down the coproduct $\psi(E)=$ $\sum E^{\prime} \otimes E^{\prime \prime}$, where $E^{\prime}$ and $E^{\prime \prime}$ are monomials in Steenrod squares. Then we observe that the Kronecker product $\left\langle\xi_{n}, E^{\prime}\right\rangle$ is non-zero only when $E^{\prime}=S q^{2^{n-1}} \cdots S q^{1}$, by definition of $\xi_{n}$. The complementary monomials $E^{\prime \prime}$ then constitute $E$ stripped by $\omega_{\left(2^{n-1}, \ldots, 1\right)}$.

Stripping is analogous to the construction of the cap product of a cohomology class $\xi$ and a homology class $X$. For an element $X$ in $\mathscr{A}$ and $\xi$ in $\mathscr{A}^{*}$, let

$$
\psi(X)=\sum X^{\prime} \otimes X^{\prime \prime}, \quad \psi(\xi)=\sum \xi^{\prime} \otimes \xi^{\prime \prime}
$$

be the usual coproducts. We shall write the action of $\xi$ on $X$ as a cap product

$$
\xi \cap X=\sum\left\langle\xi, X^{\prime}\right\rangle X^{\prime \prime}
$$

As in Subsection 4.5, we write $\widehat{X}$ for $\chi(X)$. The following stripping rules can be found, for example, in [210].

Lemma 5.3.

$$
\begin{gathered}
\xi \eta \cap X=\xi \cap(\eta \cap X), \\
\xi \cap\left(X_{1} X_{2}\right)=\sum\left(\xi^{\prime} \cap X_{1}\right)\left(\xi^{\prime \prime} \cap X_{2}\right), \\
\chi(\xi \cap X)=\chi(\xi) \cap \chi(X), \\
\langle\xi \eta, X\rangle=\langle\xi, \eta \cap X\rangle .
\end{gathered}
$$

The third rule explains how to strip 'under the hat'.

ExAmple 5.4. To strip $\widehat{S q^{7} S} q^{2}$ by $\xi_{2}$, we first write $\widehat{\xi_{2}}=\xi_{2}+\xi_{1}^{3}$, which corresponds to the stripping operation $\omega_{(2,1)}+\omega_{(1)} \omega_{(2)}$. Now

$$
\begin{gathered}
\xi_{2} \cap\left(S q^{7} S q^{2}\right)=\omega_{(2,1)}\left(S q^{7} S q^{2}\right)=S q^{5} S q^{1}, \\
\xi_{1}^{3} \cap\left(S q^{7} S q^{2}\right)=\omega_{(1)} \omega_{(2)}\left(S q^{7} S q^{2}\right)=S q^{4} S q^{2}+S q^{5} S q^{1}+S q^{6} .
\end{gathered}
$$


Hence

$$
\xi_{2} \cap\left(S \widehat{q^{7} S} q^{2}\right)=S \widehat{q^{4} S} q^{2}+\widehat{S q^{6}}=S q^{6}+S q^{4} S q^{2} .
$$

The calculation can be counter-checked in this case, because $S \widehat{q^{7} S} q^{2}=S q^{6} S q^{2} S q^{1}$, and stripping directly by $\xi_{2}=\omega_{(2,1)}$ gives $S q^{6}+S q^{4} S q^{2}$.

There is an alternative algorithm for stripping by a conjugate, which can be illustrated with reference to the above example. As we saw, stripping by $\widehat{\xi}_{2}$ is the sum of stripping by $(2,1)$ and (2)(1). Now stripping by (2)(1) is equivalent to stripping by the sum $(2,1)+(1,2)+(3)$, as can be seen by stripping first by $(2)$, and then observing that (1) can appear in front of, behind, or on top of (2). Care must be taken, however, to use only $(1,2)$ and (3) as formal stripping operators in conjunction, because individually they are not allowable vectors and do not commute with the Adem relations.

EXAMPLE 5.5. Stripping by $\widehat{\xi}_{2}$ is equivalent to stripping by the sum $(1,2)+(3)$. Hence

$$
\widehat{\xi_{2}} \cap\left(S q^{7} S q^{2}\right)=\widehat{S q^{6}}+S \widehat{q^{4} S} q^{2} .
$$

More generally, stripping by $\widehat{\xi}_{n}$ is the same as stripping by the sum of the composites of the subvectors formed by inserting markers in all possible ways in the allowable vector $\left(2^{n-1}, \ldots, 1\right)$. For example, stripping by $\widehat{\xi}_{3}$ is effected by

$$
(4)(2)(1)+(4,2)(1)+(4)(2,1)+(4,2,1) \text {. }
$$

This, in turn, is equivalent to stripping by the sum of all the vectors of the form $\left(a_{1}, a_{2}, \ldots, a_{k}\right)$ satisfying the following properties:

- each $a_{i}$ is an 'atomic' number of the form $2^{\lambda}\left(2^{\mu}-1\right)$,

- $a_{1}<a_{2}<\ldots<a_{k}$

- $\sum_{1 \leqslant i \leqslant k} a_{i}=2^{k}-1$.

Such vectors $\left(a_{1}, a_{2}, \ldots, a_{k}\right)$ are obtained from $\left(1,2, \ldots, 2^{n-1}\right)$ by inserting markers and adding up the entries between markers. For example, stripping by $\widehat{\xi}_{3}$ is equivalent to stripping by the sum of vectors

$$
(7)+(3,4)+(1,6)+(1,2,4) \text {. }
$$

Again it should be emphasised that, individually, each vector in this sum is stripped formally, and has no meaning on its own as an operation in the Steenrod algebra. Applications of conjugate stripping and related topics can be found in $[\mathbf{1 7 7}, \mathbf{1 7 8}, \mathbf{1 7 6}]$.

Stripping in the Milnor basis is very straightforward. The following result was used for the first time by Monks in [142].

Lemma 5.6. On a Milnor element $S q\left(r_{1}, r_{2}, \ldots\right)$, stripping by $\xi_{1}^{t_{1}} \xi_{2}^{t_{2}} \cdots$ gives $S q\left(r_{1}-t_{1}, r_{2}-t_{2}, \ldots\right)$. If any entry is negative, then the element is regarded as zero.

In particular, the Milnor basis is stable under stripping, and one can see immediately that, as a vector space transformation, stripping by a monomial element in $\mathscr{A}^{*}$ is a surjective map of $\mathscr{A}$, and its kernel is generated by the Milnor basis elements which are transformed to zero. For example, the kernel of stripping by $\xi_{1}$ is generated as a vector space by Milnor basis elements $S q\left(0, r_{2}, r_{3}, \ldots\right)$. 
As a simple illustration of the stripping technique, we re-work an old result of Mizuno and Saito [137], extended by Peterson [156] and further extended by Lin [117] and Li [116], which states the following relations in the Steenrod algebra.

EXAMPLE 5.7.

$$
\begin{gathered}
\sum_{0 \leqslant i \leqslant k} S q^{i} S q^{k-i}=0, \quad k \neq 0 \bmod 3, \\
\sum_{0 \leqslant i \leqslant k} S q^{i} S q^{k-i}=S q(0, k / 3), \quad k=0 \bmod 3 .
\end{gathered}
$$

Consider the vector subspace $\mathscr{V}[n]$ of $\mathscr{A}$ generated by monomials in Steenrod squares of length not greater than $n$. Let $\mathscr{V}_{k}[n]$ be the summand of $\mathscr{V}[n]$ in grading $k$. Then $\mathscr{V}[n]$ is stable under stripping and the Adem relations. The admissible elements of length not greater than $n$ form a vector space basis of $\mathscr{V}[n]$, and the Milnor product formula shows that the same is true for the Milnor basis elements. For example, $\mathscr{V}_{k}[2]$ is generated by terms $S q^{a} S q^{b}$, where $a \geqslant 2 b$ and $a+b=k$. It is also generated by $S q\left(r_{1}, r_{2}\right)$ for $r_{1}+3 r_{2}=k$. The kernel of stripping by $\xi_{1}$ is generated by $S q\left(0, r_{2}\right)$, and is therefore concentrated in gradings $k=0 \bmod 3$. In these gradings, it is generated by the single element $S q(0, k / 3)$. By examining adjacent terms in the lexicographic ordering of the admissible basis of $\mathscr{V}_{k}$ [2], one sees that

$$
S q^{k}+S q^{k-1} S q^{1}+\cdots+S q^{1} S q^{k-1}+S q^{k}
$$

is in the kernel of $\xi_{1}$. The first relation in Example 5.7 follows immediately, and the second can be proved by a further stripping argument to show that the left-hand side is non-zero. The same method can be used to demonstrate the other relations in $[116,117,156]$.

EXAMPLE 5.8 .

$$
\begin{gathered}
\sum_{0 \leqslant i \leqslant k} S q_{s}(i) S q_{s}(k-i)=0, \quad k \neq 0 \bmod 2^{s}+1, \\
\sum_{0 \leqslant i \leqslant k} S q_{s}(i) S q_{s}(k-i)=S q_{2 s}\left(k /\left(2^{s}+1\right)\right), \quad k=0 \bmod 2^{s}+1 .
\end{gathered}
$$

Another straightforward application of the stripping technique is in relation to Example 4.14.

EXAMPLE 5.9. The kernel $K$ of right multiplication by $S q^{2^{n}}$ in the Steenrod algebra is in the left ideal generated by $S q^{2^{n}}$, and $K$ is zero in degrees less than $2^{n+1}-1$.

To see why this is true, we start with a relation $E S q^{2^{n}}=0$, for some element $E$ in $\mathscr{A}(n)$, and strip by the vector $\left(2^{n}\right)$ to obtain $E=F S q^{2^{n}}$ for an element $F \in \mathscr{A}(n)$. It follows that $F S q^{2^{n}} S q^{2^{n}}=0$. If the grading of $E$ is less than $2^{n+1}-1$, then the grading of $F$ is less than $2^{n}-1$, and stripping by the vector $\left(2^{n}, 2^{n-1}\right)$ yields $F S q^{2^{n-1}}=0$. An induction argument on $n$ completes the proof.

It would be interesting to find how far stripping methods can be exploited in the analysis of ideals in $\mathscr{A}$. 
5.2 The strapping process. In [189] it is shown that there is a well-defined algebra map $\gamma: \mathscr{A} \rightarrow \mathscr{A}$ which formally divides the exponents of a string of Steenrod squares by 2 , namely

$$
\gamma\left(S q^{k_{1}} S q^{k_{2}} \cdots S q^{k_{r}}\right)=S q^{k_{1} / 2} S q^{k_{2} / 2} \cdots S q^{k_{r} / 2},
$$

with the understanding that the map is zero if any $k_{i}$ is odd. The inverse process $\gamma^{-1}$ of formally duplicating the exponents of a string of Steenrod squares is not well defined as a map of the Steenrod algebra. However, $\gamma^{-1}$ is well defined modulo the two-sided ideal generated by $S q^{1}$, as we see from the Adem relations and the equality of binomial coefficients

$$
\left(\begin{array}{c}
j-k-1 \\
i-2 k
\end{array}\right)=\left(\begin{array}{c}
2 j-2 k-1 \\
2 i-4 k
\end{array}\right) \bmod 2
$$

The situation can be partially restored in the Steenrod algebra itself by exploiting the stripping technique and the properties of the element

$$
S q(1,1, \ldots, 1)=S q^{2^{k}-1} S q^{2^{k-1}-1} \cdots S q^{3} S q^{1} .
$$

For notational convenience, we shall call this element $T^{k}$. With reference to Example 4.9 , we see that $T^{k}$ is the product of the primitives $P_{i}^{0}$, for $1 \leqslant i \leqslant k$, which generate an exterior algebra with $T^{k}$ as the top element. It can be seen immediately, from either the admissible form or the Milnor form, that $T^{k}$ is annihilated by the stripping operation $\xi^{2}$ for any element $\xi$ of positive degree in the dual Steenrod algebra.

The next result expresses the fact that for any string $E$ of $r$ even squares, the effect of pre-multiplying by $T^{k}$ is to increase the exponents of $E$ by adding on successively the numbers $2^{k}-1,2^{k-1}-1, \ldots, 3,1$ to the first $k$ exponents of $E$ (assuming $r \geqslant k$ by adding a string of $S q^{0}$ if necessary).

THEOREM 5.10. For all $r \geqslant k \geqslant 1$,

$$
T^{k} S q^{2 a_{1}} S q^{2 a_{2}} \cdots S q^{2 a_{r}}=S q^{2 a_{1}+2^{k}-1} S q^{2 a_{2}+2^{k-1}-1} \cdots S q^{2 a_{k}+1} \cdots S q^{2 a_{r}} .
$$

For example,

$$
S q^{3} S q^{1} S q^{8} S q^{8} S q^{6}=S q^{11} S q^{9} S q^{6}, \quad S q^{7} S q^{3} S q^{1} S q^{4} S q^{8} S q^{6}=S q^{11} S q^{11} S q^{7} .
$$

The proof of the result follows by induction on length, starting with the case

$$
S q^{2^{k}-1} S q^{2^{k-1}-1} \cdots S q^{3} S q^{1} S q^{2 a}=S q^{2^{k}-1+2 a} S q^{2^{k-1}-1} \cdots S q^{3} S q^{1},
$$

which can be demonstrated by Adem relations, Milnor's product formula, or checking the result when $a$ is a power of 2 and then stripping down by the vector (2). A companion result, with similar proof, applies to the case of odd exponent with a constraint on length.

THEOREM 5.11. If any exponent $a_{i}$ is odd in the range $1 \leqslant i \leqslant k$, then

$$
T^{k} S q^{a_{1}} S q^{a_{2}} \cdots S q^{a_{k}}=0 .
$$

Recalling the definition of the vector subspaces $\mathscr{V}[k]$ of $\mathscr{A}$ in the previous section, we now see how the process of formal duplication of exponents can be made to work in the Steenrod algebra. 
THEOREM 5.12. The composition

$$
\delta_{k}=T^{k} \gamma^{-1}
$$

of formal duplication and pre-multiplication by $T^{k}$ is a well-defined linear map

$$
\delta_{k}: \mathscr{V}[k] \rightarrow \mathscr{V}[k] .
$$

Furthermore, $\delta_{k}$ is injective.

The proof that $\delta_{k}$ is well defined follows from the previous two theorems by observing that $T^{k}$ kills the error terms in the process of formal duplication of exponents. Injectivity is a consequence of the fact that $\delta_{k}$ preserves the admissible basis. We refer to the maps $\delta_{k}$ as the 'strapping process'. Starting with the relation $S q^{1} S q^{1}=0$ and strapping iteratively by $\delta_{2}$ produces certain relations of the form $S q^{2 m-1} S q^{m}=0$ for arbitrarily large $m$. We have already observed that these relations are enough to generate all relations by stripping.

One consequence of Theorem 5.12 is that any relation in the Steenrod algebra can be converted into an equivalent relation involving only odd exponents by the application of $\delta_{k}$ for $k$ large enough. It is a moot point, however, whether these considerations can help in solving some of the hard questions about nilpotence heights. The papers of Monks [139] and Li [116] go further into this matter and produce more refined duplication formulae. The general idea is to combine duplication and stripping in suitable ways. For example, we note from the Adem relations that the set of strings of Steenrod squares, with all exponents odd, forms a subalgebra of the Steenrod algebra, which in Monks [139] is called $\mathcal{O}$. Furthermore, the formal length of a non-zero string of odd squares remains constant under the re-write rules of the Adem relations. This allows us to define a map

$$
\lambda: \mathcal{O} \rightarrow \mathcal{O}
$$

by the prescription 'double the exponent and subtract 1':

$$
\lambda\left(S q^{a_{1}} S q^{a_{2}} \cdots S q^{a_{k}}\right)=S q^{2 a_{1}-1} S q^{2 a_{2}-1} \cdots S q^{2 a_{k}-1} .
$$

This map can alternatively be described as the composite of three maps, namely formal duplication $\gamma^{-1}$, followed by pre-multiplication with $T^{k}$, followed by stripping with $\xi_{k}^{2}$. On a Milnor basis element $S q\left(r_{1}, r_{2}, \ldots, r_{k}\right)$, the effect of $\lambda$ is to produce $S q\left(2 r_{1}+1,2 r_{2}+1, \ldots, 2 r_{k-1}+1,2 r_{k}-1\right)$ as explained in Monks [139] (note that the -1 in the end position comes from stripping by $\xi_{k}^{2}$ ). The algebra $\mathcal{O}$ is generated by Milnor elements with odd entries. Clearly, $\lambda$ is formally multiplicative and therefore actually multiplicative as map on $\mathcal{O}$. It is also a monomorphism, because it sends distinct admissibles to distinct admissibles. Monks [139] concludes that $S q^{k}$ and $S q^{2 k-1}$ have the same nilpotence height when $k$ is odd. More generally, we have the following result.

THEOREM 5.13. For a sequence $a_{1}, a_{2}, \ldots, a_{k}$ of odd numbers, the monomials

$$
S q^{a_{1}} S q^{a_{2}} \cdots S q^{a_{k}}, \quad S q^{2 a_{1}-1} S q^{2 a_{2}-1} \cdots S q^{2 a_{k}-1}
$$

have the same nilpotence height. A similar statement applies to $S q\left(r_{1}, r_{2}, \ldots, r_{k}\right)$ and $S q\left(2 r_{1}+1,2 r_{2}+1, \ldots, 2 r_{k-1}+1,2 r_{k}-1\right)$. 


\subsection{Problems}

Problem 5.14. Can the Steenrod algebra be developed from the basic relations

$$
S q^{2^{k}-1} S q^{2^{k-1}}=0
$$

by incorporating the stripping process as part of the structure?

Problem 5.15. Can the Steenrod algebra be developed from the basic relation

$$
S q^{1} S q^{1}=0
$$

by incorporating the stripping and strapping processes as part of the structure?

Problem 5.16. How can analogues of the stripping and strapping processes be developed in the differential operator algebra $\mathscr{D}$ and modulo $p$ reductions?

Problem 5.17. Working in the symmetric function algebra, can the stripping technique be used to elucidate any aspects of the combinatorics of Young diagrams and the representations of symmetric groups, for example the theory of $p$-hooks and the Littlewood-Richardson rule?

Problem 5.18. How can the stripping technique and the Z-basis be exploited to relate the kernel of right multiplication in the Steenrod algebra by $S q^{2^{n}}$ and the left ideal generated by $S q^{2^{n+1}}-1$ ?

\section{Iteration theory and nilpotence}

The purpose of this section is to draw a parallel between some of the elementary aspects of the study of iteration theory of analytic functions in the plane and the global method of producing relations in the Steenrod algebra by the use of formal power series. The comparison is purely analogical. At present we cannot claim any serious interconnections between the two areas. We shall then contrast the global procedures with local procedures for tackling nilpotence results using the stripping technique.

6.1 Iteration of power series. It is clear from Theorem 2.17 and Example 3.4 that composites and functional inverses of quadratic polynomials of the form $z+t z^{2}$ have a role to play in finding composites and conjugates of Steenrod squares. From the point of view of complex dynamics [22], the three quadratic polynomials

$$
z+t z^{2}, \quad z+z^{2}, \quad z^{2}+1 / 4,
$$

for $t \neq 0$, are equivalent because they are conjugate via Möbius transformations, and this implies, for example, that the intrinsic geometry of their Julia sets is the same. In particular, the parameter $t$ can be taken equal to 1 . In discussing Julia sets and the Mandelbrot set associated with the iteration theory of quadratic polynomials, it is customary to take the quadratic polynomial in standard form $z^{2}+c$, for a complex parameter $c$, which can always be achieved by Möbius conjugation. For our purposes, however, it is more convenient to take the form $z+t z^{2}$ for a quadratic polynomial, and more generally

$$
t_{0} z+t_{1} z^{2}+\cdots+t_{n} z^{n+1}+\cdots
$$


for a power series, where the origin is then a fixed point and $t_{0}$ is a dynamic invariant, the derivative at the fixed point, whose modulus measures whether the fixed point is attracting $\left(\left|t_{0}\right|<1\right)$, repelling $\left(\left|t_{0}\right|>1\right)$ or indifferent $\left(\left|t_{0}\right|=1\right)$. We shall restrict attention to the case $t_{0}=1$. The set of all formal power series

$$
f(z)=z+t_{1} z^{2}+\cdots+t_{n} z^{n+1}+\cdots
$$

forms a group under composition (formal substitution). The product and inverse are expressed in Theorem 2.17 by the convolution product $\mathbf{t} * \mathbf{S}$ and conjugate $\hat{\mathbf{t}}$ of formal sequences $\mathbf{t}, \mathbf{s}$.

Given any sequence $\mathbf{f}=\left(f_{0}, f_{1}, f_{2}, \ldots\right)$ of set functions $f_{i}: \mathbf{C} \rightarrow \mathbf{C}$, the bound set $B(\mathbf{f})$ is defined to be the set of complex numbers $z$ such that the sequence $\left(f_{0}(z), f_{1}(z), f_{2}(z), \ldots\right)$ is bounded; in other words,

$$
B(\mathbf{f})=\left\{z \in \mathbf{C}: \sup _{n}\left|f_{n}(z)\right|<\infty\right\} .
$$

For example, if $f(z)$ is a polynomial and the sequence is defined recursively by iteration,

$$
\begin{gathered}
f_{0}(z)=z, \quad f_{1}(z)=f(z), \\
f_{n}(z)=f\left(f_{n-1}(z)\right),
\end{gathered}
$$

then the bound set is called the filled-in Julia set, $B(\mathbf{f})=K(f)$, of the polynomial $f(z)$. The boundary, in the sense of point set topology, is the actual Julia set $J(f)=\partial K(f)$ (this is not a valid definition for arbitrary analytic functions). If the sequence $f_{n}$ is defined recursively by

$$
\begin{gathered}
f_{0}(z)=z, \quad f_{1}(z)=z+z^{2}, \\
f_{n}(z)=z+\left(f_{n-1}(z)\right)^{2}
\end{gathered}
$$

(which is not an iteration sequence), then the corresponding bound set is called the Mandelbrot set, $B(\mathbf{f})=M$. The parameter $c$ lies in $M$ if and only if the Julia set of $z^{2}+c$ is connected; otherwise, the Julia set is a Cantor set. The picture of $M$ is very familiar [154]. It is a compact connected subset of the plane whose intersection with the real axis is the interval $[-2,1 / 4]$. For example, the point $c=1 / 4$ corresponds to the quadratic polynomial $z+z^{2}$, whose Julia set is therefore connected. Although the quadratic polynomials $z+z^{2}$ and $z+t z^{2}$ are equivalent, for $t \neq 0$, as far as dynamics is concerned, there is a good reason for retaining the parameter $t$ in the study of formal iteration theory. Indeed, we can consider the quadratic polynomials $q_{n}(z)=z+t_{n} z^{2}$, for $n=1,2, \ldots$, and form the sequence

$$
\begin{gathered}
p_{0}(z)=z, \quad p_{1}(z)=q_{1}(z)=z+t_{1} z^{2}, \\
p_{n}(z)=q_{n}\left(p_{n-1}(z)\right) .
\end{gathered}
$$

The actual iteration sequence of $z+z^{2}$ is obtained by putting all $t_{i}$ equal to 1 . For a selection of values of $t_{i}$ near to 1 , the bound set $B(\mathbf{p})$ of this sequence provides a deformation of the filled-in Julia set of $z+z^{2}$. It is interesting to study the geometry of $B(\mathbf{p})$ in the spirit of complex dynamics [32]. Of course, the construction of iterated composition may be made in the formal sense on any sequence of formal sequences $\mathbf{t}_{1}, \mathbf{t}_{2}, \ldots, \mathbf{t}_{n}, \ldots$ The result is expressed by the sequence of iterated convolution products $\mathbf{t}_{1} * \mathbf{t}_{2} * \cdots * \mathbf{t}_{n}$. In particular, the case $\mathbf{t}_{n}=\left(t_{n}, 0, \ldots\right)$, where all terms of the formal sequence are zero except the first, corresponds to the quadratic case. For this particular choice we have the following formulae. 
EXAMPLE 6.1.

$$
\begin{gathered}
\mathbf{t}_{1} * \mathbf{t}_{2}=\left(t_{1}+t_{2}, 2 t_{1} t_{2}, t_{1} t_{2}^{2}\right), \\
\mathbf{t}_{1} * \mathbf{t}_{2} * \mathbf{t}_{3}=\left(t_{1}+t_{2}+t_{3}, 2\left(t_{1} t_{2}+t_{1} t_{3}+t_{2} t_{3}\right), t_{1}^{2} t_{2}+t_{1}^{2} t_{3}+t_{2}^{2} t_{3}+6 t_{1} t_{2} t_{3},\right. \\
\left.6 t_{1}^{2} t_{2} t_{3}+4 t_{1} t_{2}^{2} t_{3}, 6 t_{1}^{2} t_{2}^{2} t_{3}+2 t_{1}^{3} t_{2} t_{3}, 4 t_{1}^{3} t_{2}^{3} t_{3}, t_{1}^{4} t_{2}^{2} t_{3}\right) .
\end{gathered}
$$

The modulo 2 reductions of these examples are

$$
\left(t_{1}+t_{2}, 0, t_{1} t^{2}\right), \quad\left(t_{1}+t_{2}+t_{3}, 0, t_{1}^{2} t_{2}+t_{1}^{2} t_{3}+t_{2}^{2} t_{3}, 0,0,0, t_{1}^{4} t_{2}^{2} t_{3}\right),
$$

and it follows by induction on $n$ that all terms in the modulo 2 reduction of $\mathbf{u}=\mathbf{t}_{1} * \mathbf{t}_{2} * \cdots * \mathbf{t}_{n}$ vanish except in positions $2^{k}-1$, where the term $u_{2^{k}-1}$ is the weighted elementary symmetric function [116], obtained from the elementary symmetric function

$$
\sum_{i_{1}<i_{2}<\ldots<i_{k}} t_{i_{1}} t_{i_{2}} \cdots t_{i_{k}}
$$

in $n \geqslant k$ variables $t_{i}$, written in increasing order of subscript, by replacing $t_{i}$ with $t_{i}^{k^{k-i}}$.

Lemma 6.2. The modulo 2 reduction of $u_{2^{k}-1}$ is

$$
\sum_{i_{1}<i_{2}<\ldots<i_{k}} t_{i_{1}}^{2^{k-1}} t_{i_{2}}^{2^{k-2}} \cdots t_{i_{k}}
$$

From Theorem 2.17, we then have the global product formula in the Steenrod algebra [116]:

$$
\begin{aligned}
\left(\sum_{i \geqslant 0} t_{1}^{i} S q^{i}\right) \cdots\left(\sum_{i \geqslant 0} t_{n}^{i} S q^{i}\right) & =\exp _{\vee}\left(t_{1} D_{1}\right) \circ \exp _{\vee}\left(t_{2} D_{1}\right) \circ \cdots \circ \exp _{\vee}\left(t_{n} D_{1}\right) \\
& =\exp _{\vee}\left(u_{1} D_{1}+u_{3} D_{3}+\cdots+u_{2^{n}-1} D_{2^{n}-1}\right) \\
& =\exp _{\vee}\left(u_{1} D_{1}\right) \vee \cdots \vee \exp _{\vee}\left(u_{2^{n}-1} D_{2^{n}-1}\right) \\
& =\left(\sum_{i \geqslant 0} u_{1}^{i} D\left(1^{i}\right)\right) \vee \cdots \vee\left(\sum_{i \geqslant 0} u_{2^{n}-1}^{i} D\left(2^{n}-1\right)^{i}\right) .
\end{aligned}
$$

In principle, it should be possible to pick off the coefficient of $t_{1}^{r_{1}} \cdots t_{n}^{r_{n}}$ in the expansion of the right-hand side of this formula, and thereby obtain an expression for the iterated product

$$
S q^{r_{1}} S q^{r_{2}} \cdots S q^{r_{n}}
$$

in terms of the Milnor basis elements $D\left(1^{i_{1}} 3^{i_{2}} \cdots\left(2^{n}-1\right)^{i_{n}}\right)$. In practice, it seems a difficult task to find the parity of the relevant coefficients in general. For example, knowledge of the parity of the coefficient of $\left(t_{1} t_{2} \cdots t_{n}\right)^{k}$ for all $n$ and $k$ would settle the nilpotence question for the Steenrod squaring operations $S q^{k}$. In [116], Li goes some way to solving this problem in special cases, and recaptures results in Monks' work [143] on nilpotence problems for odd exponents.

In some sense, therefore, nilpotence in the Steenrod algebra has to do with the iterated composition of deformations $z+t z^{2}$ of the quadratic polynomial $z+z^{2}$ corresponding to the point $1 / 4$ in the Mandelbrot set. In passing from power series to differential operators, we make the umbral substitution of $D_{k}$ for $z^{k+1}$. It would 
be interesting to know if there could be any significant interplay between complex dynamics and the Steenrod algebra.

6.2 Nilpotence in the Steenrod algebra. For certain families of elements in the Steenrod algebra, the nilpotence height is known [116, 139, 209]. For example, the Wilson conjecture has been proved.

THEOREM 6.3 (Walker and Wood [209]). The nilpotence height of $S q^{2^{k}}$ is $2 k+2$.

Previously, it was shown by Don Davis $[\mathbf{5 5}, \mathbf{5 7}]$ that $\left(S q^{2^{k}}\right)^{2 k+1} \neq 0$. This can be seen directly by evaluation on a monomial in two variables in the polynomial algebra $\mathbf{F}_{2}[x, y]$.

THEOREM 6.4 (Walker and Wood [212]).

for $k \geqslant 1$.

$$
\left(S q^{2^{n}}\right)^{2 n+1}\left(x y^{k 2^{n+1}-1}\right)=\left[\left(S q^{2^{n-1}}\right)^{2 n-1}\left(x y^{(k+1) 2^{n}-1}\right)\right]^{2}
$$

The odd prime analogue of Theorem 6.3 is also proved in the same paper.

THEOREM 6.5 (Walker and Wood [212]). The nilpotence height of $P^{p^{n}}$ is $p(n+1)$.

Monks has generalised Theorem 6.4 to the $P_{t}^{s}$ family for the prime 2 in [139], and Karaca has extended this to the odd prime case.

TheOREM 6.6 (Karaca [94]). In the modulo $p$ Steenrod algebra, the nilpotence height of $P_{t}^{s}$ is

$$
p[s / t]+p
$$

The work of Monks contains further information on nilpotence heights of squares with odd exponent and Milnor basis elements. A sample result is quoted here.

TheOREM 6.7 (Monks [139]). The height of $S q^{2^{n}-1}$ is $n+1$.

One way to prove this result is to notice that $\left(S q^{2^{n}-1}\right)^{n+1}$ lies in the Hopf subalgebra $\mathscr{A}(n-1)$ and can therefore be reduced to Y-basis form as in Theorem 4.12. In such a reduction, the number of odd squares in any monomial does not decrease [220]. But any Y-basis element for $\mathscr{A}(n-1)$ has at most $n$ odd squares in it, hence the result. The argument extends to show that any monomial of Steenrod squares in $\mathscr{A}(n-1)$ containing more than $n$ odd factors is zero. In particular, the nilpotence height of $S q^{2 k+1}$ does not exceed the smallest number $r$ for which $2 k+1<2^{r}-1$. In general, an upper bound for the nilpotence height of an element can be given in terms of the smallest finite subalgebra containing the element, but this is not easy to determine for an arbitrary string of Steenrod squares.

It would be nice to exploit stripping techniques to find the heights of all the atomic squares $S q^{2^{m}\left(2^{k}-1\right)}$. The cases $m=0$ and $k=0$ are two special cases in the spectrum of atomic numbers of the form $2^{m}\left(2^{k}-1\right)$ for which results are known, but the methods of proof are very different. It is not clear, for example, how one should 
tackle the case of exponent $2\left(2^{k}-1\right)$. The following table indicates the heights of the first few atomic squares arranged in the same pattern as the $P_{t}^{s}$ in the table of Example 4.7. The entry at position $(k, m)$ is the height of $S q^{2^{m}\left(2^{k}-1\right)}$.

\begin{tabular}{l|c|c|c|c|c}
4 & 10 & & & & \\
\cline { 2 - 7 } 3 & 8 & 8 & & & \\
\cline { 2 - 7 } 2 & 6 & 7 & 8 & & \\
\cline { 2 - 7 } 1 & 4 & 4 & 5 & 6 & \\
\cline { 2 - 7 } 0 & 2 & 3 & 4 & 5 & 6 \\
\cline { 2 - 7 } & \multicolumn{1}{c}{1} & 2 & 3 & 4 & 5
\end{tabular}

There is another direction in which nilpotence questions can be generalised. Let $L_{n}$ denote the left ideal in the Steenrod algebra generated by $\mathscr{A}(n)^{+}$. For example, $L_{0}$ is the set of elements in the Steenrod algebra which can be written in the form $E S q^{1}$ for some element $E$ in $\mathscr{A}$. Define $L_{r}$ to be 0 for $r \leqslant-1$. Experimental evidence suggests the following ideal conjecture.

CONJECTURE 6.8. $\left(S q^{2^{n}}\right)^{2 k} \in L_{n-k}$.

The proof of the conjecture is straightforward for small values of $k$ and all $n$, but gets progressively more difficult as $k$ increases. At one time, the intention was to prove the height conjecture Theorem 6.3 by induction through the ideal conjecture, but now we are in the curious position of knowing the two ends of the ideal conjecture but nothing in between!

A criterion for belonging to $L_{n}$ is easily established by the stripping technique.

Lemma 6.9. An element $E$ in the Steenrod algebra belongs to the ideal $L_{n}$ if and only if it is right annihilated by the top element of $\mathscr{A}(n)$.

The ideal conjecture states, in particular, the following result.

CONJeCtuRe 6.10. $\left(S q^{2^{n}}\right)^{2 n} S q^{1}=0$.

The truth of this statement leads to the nilpotence result Theorem 6.3 by stripping by the vector (1) and pre-multiplying by $\left(S q^{2^{n}}\right)^{2}$. It would be interesting to prove the converse.

6.3 Annihilator ideals. The non-vanishing of $\left(\mathrm{Sq}^{2^{k}}\right)^{2 k+1}$ cannot be detected on polynomials in one variable because, for any positively graded element $E$ in the Steenrod algebra, $E^{2}$ annihilates all polynomials in a single variable. An argument in [73], prior to the general proof, had shown directly that $\left(S q^{2^{k}}\right)^{2 k+2}$ vanishes on polynomials in two variables. This raises the general question of how few variables are needed to detect a non-zero element in the Steenrod algebra. To be more precise, consider the two-sided ideal $\mathscr{K}(n)$ of all elements of the Steenrod algebra which annihilate $\mathbf{F}_{2}\left[x_{1}, \ldots, x_{n}\right]$. How do we describe the quotient $\mathscr{A} / \mathscr{K}(n)$ ? This problem is addressed in [74].

The problem can also be formulated in the divided differential operator algebra. 
We recall that $\mathscr{D}$ preserves the polynomial algebra $\mathbf{W}(n)$. Although $\mathscr{D}$ has no nilpotent elements, there are elements of $\mathscr{D}$ which annihilate all polynomials in a fixed number of variables. For example, $D_{1} \vee D_{3}-D_{2} \vee D_{2}$ annihilates all polynomials in one variable. We can ask for a description of the two-sided ideal in $\mathscr{D}$ which annihilates $\mathbf{W}(n)$. Even the case $n=1$ is interesting. Here, the operator $D_{k}$ boils down to

$$
x^{k+1} \frac{d}{d x} \text {. }
$$

The wedge product is given by

$$
D_{k} \vee D_{l}=x^{k+l+2} \frac{d^{2}}{d x^{2}} .
$$

In the modulo 2 case, we note from Theorem 6.4 that $\left(S q^{2^{n}}\right)^{2 n+1}$ is non-zero on $\mathbf{W}(2) \otimes \mathbf{F}_{2}$, and this may be viewed as a measure of the depth of the nilpotence result. We refer to $[\mathbf{7 3}, \mathbf{7 4}]$ for recent results in this area.

\subsection{Problems.}

Problem 6.11. Find the heights of all the atomic squares $S q^{2^{m}\left(2^{k}-1\right)}$.

Problem 6.12. Prove the ideal conjecture $\left(S q^{2^{n}}\right)^{2 k} \in L_{n-k}$, in particular the case $k=n$.

Problem 6.13. Given a non-zero element $E$ in the Steenrod algebra, what is the smallest number, $n$, of variables such that $E$ is detected by its action on $\mathbf{F}_{2}\left[x_{1}, \ldots, x_{n}\right]$ ? For this value of $n$, is there a detecting monomial of degree equal to the excess of $E$ ?

Problem 6.14. Investigate the quotients of subalgebras of $\mathscr{D}$ by the annihilators of all polynomials in a fixed number of variables, in the integral and modular cases.

Problem 6.15. Find some significant connection between complex dynamics of quadratic functions and the Steenrod algebra.

\section{The hit problem and invariant theory}

The expression 'hit problem' is used here to refer to certain special cases of a general problem in algebra which has many applications to topology. Let $M=$ $\bigoplus_{i \geqslant 0} M^{i}$ be a graded left module over a graded ring $R=\bigoplus_{i \geqslant 0} R^{i}$. We wish to find a criterion for an element $f \in M^{n}, n>0$, to be expressible in the form

$$
f=\sum_{1 \leqslant i \leqslant k} r_{i} f_{i},
$$

where $r_{i} \in R$, and the $f_{i} \in M$ have strictly lower grading than that of $f$. Such elements form a submodule $H=R^{+} M$, and have become known as 'hit' elements. If $M=R^{+}$is viewed as a left module over $R$, then the problem is to find the decomposables in $R$. For example, all Steenrod squares are decomposable except for the $S q^{2^{n}}$. This fact itself has had interesting applications in the early applications 
of the Steenrod algebra in topology, as we observed in the introductory section. A closely related problem is to find an additive basis of the quotient

$$
C=M / H
$$

of $M$ by the hit elements $H$. Representatives of such a basis then furnish a minimal generating set for $M$ as an $R$-module. Finding minimal generating sets for modules is a recurrent problem in the calculation of the cohomology of algebras by minimal resolution techniques, as, for example, in the Adams spectral sequence [145]. We shall use the letter $C$ to refer generically to the quotient $M / H$ for the hit problem associated with an action of a ring on a module $M$. In the first instance, $C$ is a graded abelian group. It is a vector space if $R$ is an algebra over a field.

7.1 The equivariant version for differential operators. To add more structure to the hit problem, we consider an equivariant version in which a group $G$ acts on the right of each $M^{i}$ compatibly with the left action of $R$. In other words, each $M^{i}$ is a representation of $G$, and an element $r \in R^{n}$ induces a $G$-map $M^{i} \rightarrow M^{i+n}$. The hit problem is then equivariant. An element $f \in M$ is hit if and only if $f \gamma$ is hit for $\gamma \in G$. Furthermore, $C$ is a representation of $G$, which is of some interest in group theory. The following well-known example is taken from classical invariant theory [183].

EXAMPLE 7.1. Let $M=\mathbf{W}(n) \otimes \mathbf{Q}=\mathbf{Q}\left[x_{1}, \ldots, x_{n}\right]$ be the polynomial algebra over the rationals in $n$ variables, graded by degree as usual. Let $G=\Sigma_{n}$ be the symmetric group acting on the right of a polynomial $f\left(x_{1}, \ldots, x_{n}\right)$ by

$$
(f \gamma)\left(x_{1}, \ldots, x_{n}\right)=\left(x_{\gamma(1)}, \ldots, x_{\gamma(n)}\right),
$$

where $\gamma \in \Sigma_{n}$. Take for $R$ the ring of symmetric polynomials in $M$ acting on $M$ by the usual multiplication of polynomials. The $R$-action commutes with the $G$-action. The quotient $C$ in this case is known [183] to be isomorphic as a $G$-space to the regular representation of $\Sigma_{n}$. For example, in the case $n=3$, the equivalence classes of the monomials

$$
1, \quad x_{1}, \quad x_{2}, \quad x_{1}^{2}, \quad x_{2}^{2}, \quad x_{1}^{2} x_{2}
$$

generate $C$. From elementary representation theory, it is then known that the three irreducible representations of $\Sigma_{3}$ must appear in $C$ with multiplicity equal to their dimension. Indeed, the trivial representation appears once, generated by 1 in grading 0 . The sign representation appears once, generated by $x_{1}^{2} x_{2}$ in grading 3 , and the irreducible 2-dimensional representation of $\Sigma_{3}$ appears twice, generated by $x_{1}, x_{2}$ in grading 1 and by $x_{1}^{2}, x_{2}^{2}$ in grading 2 .

In this example, every homogeneous polynomial $f$ of degree at least 4 is hit; in other words, it can be written in the form

$$
f=r_{1}+r_{2} x_{1}+r_{3} x_{2}+r_{4} x_{1}^{2}+r_{5} x_{2}^{2}+r_{6} x_{1}^{2} x_{2},
$$

where the $r_{i}$ are symmetric polynomials of positive degree. To verify this statement, it is enough to check that every monomial $f$ of degree 4 is hit, because the product of a hit element by any polynomial is also hit. If $f$ is divisible by $x_{1} x_{2} x_{3}$, then it is hit. Furthermore, $x_{i}^{3}$ is hit because $x_{i}$ satisfies the equation

$$
\left(X-x_{1}\right)\left(X-x_{2}\right)\left(X-x_{3}\right)=0 .
$$


By equivariance, it remains to verify that the monomial $x_{1}^{2} x_{2}^{2}$ is hit. The formula

$$
x_{1}^{2} x_{2}^{2}=x_{1} x_{2}\left(x_{1} x_{2}+x_{1} x_{3}+x_{2} x_{3}\right)-x_{1} x_{2} x_{3}\left(x_{1}+x_{2}\right)
$$

shows that $x_{1}^{2} x_{2}^{2}$ is hit, and an actual representation in terms of the basis is given by

$$
x_{1}^{2} x_{2}^{2}=-e_{2}^{2}-e_{2} x_{1}^{2}-e_{2} x_{2}^{2}+\left(e_{1} e_{2}-e_{3}\right) x_{1}+\left(e_{1} e_{2}-e_{3}\right) x_{2},
$$

where the $e_{i}$ are the elementary symmetric functions.

The general case of $n$ variables is worked out by Artin in [18]. The equivalence classes of the $n$ ! monomials $x_{1}^{i_{1}} x_{2}^{i_{2}} \cdots x_{n-1}^{i_{n-1}}$, where $0 \leqslant i_{r} \leqslant r$, form the Artin basis of the regular representation $C$ of the symmetric group. All homogeneous polynomials of degree greater than $n(n-1) / 2$ are hit. Of course, this is just one example of the fundamental result in classical invariant theory which states that the set of co-invariants $C$ is a finite dimensional vector space over the rationals [183].

The success in answering the hit problem in the above example is partly due to commutativity of the ring of operators and the fact that the product of a hit element by a polynomial is also hit. This will not be true in general. In the next example, $M$ and $G$ remain the same, but the ring of operators is changed to $R=\mathscr{D} \otimes \mathbf{Q}$, the differential operator algebra over the rationals.

EXAMPLE 7.2. Recall that $\mathscr{D} \otimes \mathbf{Q}$ is generated under composition by the operators

$$
D_{k}=\sum_{i \geqslant 1} x_{i}^{k+1} \frac{\partial}{\partial x_{i}},
$$

and $D_{1}, D_{2}$ form a minimal algebraic generating set. By the symmetry of the $D_{k}$, it is evident that the action of $\mathscr{D} \otimes \mathbf{Q}$ on $M=\mathbf{W}(n) \otimes \mathbf{Q}$ commutes with the action of the symmetric group. The hit problem in this case reduces to the question of finding criteria on a polynomial $g$ such that the differential equation

$$
D_{1} f_{1}+D_{2} f_{2}=g
$$

can be solved for polynomials $f_{1}, f_{2}$.

In the case $n=1$, the equation $D_{k}(x)=x^{k}$ shows immediately that all powers of $x$ are hit except $x$ itself. In the two-variable case, it can be shown that $1, x_{1}, x_{2}, x_{1} x_{2}, x_{1}^{2} x_{2}$ form a basis of the quotient $C$. In particular, the quotient is finite dimensional, as in Example 7.1. Furthermore, the differential equation $D_{1} f_{1}+D_{2} f_{2}=g$ can be solved for any homogeneous polynomial $g$ of degree at least 4 . Another similarity with Example 7.1 is that the monomials $x_{1} x_{2}, x_{1}^{2} x_{2}$ generate the regular representation of $\Sigma_{2}$ in $C$. The monomial $x_{1} x_{2}$ generates the trivial representation, and the equation $D_{1}\left(x_{1} x_{2}\right)=x_{1}^{2} x_{2}+x_{1} x_{2}^{2}$ shows that $x_{1}^{2} x_{2}$ generates the sign representation of $\Sigma_{2}$ in $C$.

In the case of three variables, $n=3$, it is shown in [211] that the equivalence classes of the monomials

$$
\begin{aligned}
& 1, \quad x_{1}, \quad x_{2}, \quad x_{3}, \quad x_{1} x_{2}, \quad x_{1} x_{3}, \quad x_{2} x_{3}, \quad x_{1}^{2} x_{2}, \quad x_{1}^{2} x_{3}, \quad x_{2}^{2} x_{3}, \\
& x_{1} x_{2} x_{3}, \quad x_{1} x_{2}^{2} x_{3}, \quad x_{1} x_{2} x_{3}^{2}, \quad x_{1} x_{2}^{2} x_{3}^{2}, \quad x_{1} x_{2} x_{3}^{3}, \quad x_{1} x_{2}^{2} x_{3}^{3}
\end{aligned}
$$

generate $C$. The regular representation is generated by those monomials in the list which are divisible by $x_{1} x_{2} x_{3}$. This time, the differential equation $D_{1} f_{1}+D_{2} f_{2}=g$ can be solved if the homogeneous polynomial $g$ has degree at least 7 . In the general 
case of $n$ variables, it is known that $C$ is finite dimensional. However, the following conjecture, suggested by the above particular cases, seems harder to prove.

CONJECTURE 7.3. In the usual action of the differential operator algebra $\mathscr{D} \otimes \mathbf{Q}$ on the polynomial algebra $\mathbf{W}(n) \otimes \mathbf{Q}=\mathbf{Q}\left[x_{1}, \ldots, x_{n}\right]$, the quotient $C$ of $\mathbf{W}(n) \otimes \mathbf{Q}$ by the hit elements contains the regular representation of the symmetric group $\Sigma_{n}$ generated by the equivalence classes of monomials divisible by the product of the variables $x_{1} \cdots x_{n}$. In particular, the highest grading of $C$ is $d=n(n+1) / 2$ and, in this grading, $C_{d}$ is the 1-dimensional sign representation of $\Sigma_{n}$, generated by the equivalence class of $x_{1} x_{2}^{2} \cdots x_{n}^{n}$. Furthermore, the equivalence classes of monomials of the form

$$
x_{1}^{i_{1}} x_{2}^{i_{2}} \cdots x_{n}^{i_{n}},
$$

where $1 \leqslant i_{r} \leqslant r$, form a basis of the part of $C$ divisible by $x_{1} \cdots x_{n}$.

This conjecture implies, in particular, that every homogeneous polynomial $f$ of degree greater than $n(n+1) / 2$ is hit; in other words, the differential equation

$$
D_{1} f_{1}+D_{2} f_{2}=g
$$

can be solved for any $g$ in these degrees. There is clearly a close connection between the representation theory of the symmetric group and the hit problem for the differential operator algebra. The decomposition of $\mathbf{W} \otimes \mathbf{Q}$ by a complete set of orthogonal idempotents associated with the irreducible representations of $\Sigma_{n}$ is preserved by the action of $\mathscr{D} \otimes \mathbf{Q}$. The piece of $\mathbf{W} \otimes \mathbf{Q}$ corresponding to the trivial representation is the subspace of symmetric polynomials.

THEOREM 7.4. Any symmetric polynomial divisible by $x_{1} \cdots x_{n}$ and of degree greater than $n$ is a hit element by a differential operator in $\mathscr{D}$.

This follows by restricting the algebraic Thom map to $n$ variables. The behaviour of the differential operator algebra on the polynomials corresponding to the other representations of the symmetric group would seem to be more intricate.

To account for polynomials not divisible by the product of the variables, it is useful to note that the action of the differential operator algebra $\mathscr{D}$ on polynomials commutes with the action of the larger semigroup $\Gamma_{n}$ of functions

$$
\gamma:\{0,1, \ldots, n\} \rightarrow\{0,1, \ldots, n\}
$$

satisfying the condition $\gamma(0)=0$. The product in this semigroup is composition of functions, and the symmetric group $\Sigma_{n}$ is a subgroup of $\Gamma_{n}$ in a natural way. The right action of $\Gamma_{n}$ on polynomials is defined as for the symmetric group, with the understanding that $x_{0}=0$. This allows variables to be equated to each other or to be put to zero. We refer to [211] for work on the rational hit problem for differential operators with this extended semigroup of symmetries.

Conjecture 7.3 can be re-cast in its dual form, in terms of the contragredient down operators introduced in Subsection 2.3.

CONJECTURE 7.5. The intersection of the kernels of the down operators $D_{1}^{*}$ and $D_{2}^{*}$ on the divided polynomial algebra $\mathbf{W}^{*}(n)$ is zero in degrees above $n(n+1) / 2$.

A useful lemma, referred to as the ' $\chi$-trick' $[152,218,219]$, that facilitates the 
proof of many results on hit problems is formulated here in terms of the differential operator algebra $\mathscr{D}$, acting in the usual way on polynomials.

THEOREM 7.6. Let $E$ be an element in $\mathscr{D}$, with composition conjugate $\hat{E}=\chi(E)$. Let $u, v \in \mathbf{W}$ be two polynomials. Then $v \hat{E}(u)-u E(v)$ is hit.

By linearity, it is sufficient to prove the result for $E=D(P)$, where $P$ is a multiset. The proof relies on Lemmas 2.18 and 2.19. We start with the formula

$$
\sum_{K \cup L=M} D(K)(u) D(L)(v)=D(M)(u v),
$$

and replace $v$ by $\chi(D(N))(v)$, where $N$ is a multiset, to obtain

$$
\sum_{K \cup L=M} D(K)(u) D(L)(\chi(D(N))(v))=D(M)(u \chi(D(N))(v)) .
$$

We now sum over multisets $M, N$ such that $M \cup N=P$, to obtain

$$
\sum_{K \cup L \cup N=P} D(K)(u) D(L)(\chi(D(N))(v))=\sum_{M \cup N=P} D(M)(u \chi(D(N))(v)) .
$$

On the right-hand side, all terms are hit elements, except possibly $u \chi(D(P))(v)$, arising from the empty multiset $M$. In the light of the formula

$$
\sum_{K \cup L=M} D(K) \circ \chi(D(L))=0,
$$

all terms on the left-hand side also vanish, except $D(P)(u)(v)$, arising from the case where $L, N$ are both empty. This completes the proof of Theorem 7.6.

A typical application of the $\chi$-trick gives a criterion for a hit element in terms of excess.

THEOREM 7.7. Let $E$ be an element in $\mathscr{D}$ in positive grading such that the excess of $\chi(E)$ exceeds the degree of the homogeneous polynomial $u$. Then, for any homogeneous polynomial $v$ of positive degree, the element $u E(v)$ is hit.

The proof follows immediately from Theorem 7.6 and the definition of excess in Lemma 4.1

7.2 Modular hit problems. The hit problem for the differential operator algebra $\mathscr{D}$ acting on $\mathbf{W}$ can, in principle, be posed over the integers, but it would then seem to be a very difficult question to answer in more than a few variables. On the other hand, modular reductions of the integral question lead back to the original problem, in the context of the Steenrod algebra, posed by Frank Peterson [158]. Several reasons for studying the hit problem are listed in [219]. These include Frank Peterson's work on Stiefel-Whitney classes and bordism of manifolds [158, 159], Bill Singer's work [181] on covariants of the general linear group $\operatorname{GL}\left(n, \mathbf{F}_{2}\right)$ with applications to the Adams spectral sequence, and the relationship between the modular representation theory of the general linear groups over finite fields with the stable splitting of the classifying spaces $B(\mathbf{Z} / p)^{n}$ and $B\left(T^{n}\right)[\mathbf{7 9}, \mathbf{1 3 6}, \mathbf{2 1 6}]$. In the case of the Steenrod algebra, the problem is to find criteria for a homogeneous 
polynomial $f$ in $\mathbf{W}_{d}(n) \otimes \mathbf{F}_{2}$ to be of the form

$$
f=\sum_{i>0} S q^{i} f_{i},
$$

for some polynomials $f_{i}$ of degree $d-i$. Recall that the Steenrod squares are given in terms of differential operators by $S Q^{r}=D\left(1^{r}\right)$. The Steenrod algebra is a subalgebra of $\mathscr{D} \otimes \mathbf{F}_{2}$, and a prior question, therefore, is to ask for the solution of the hit problem for the ring of operators $\mathscr{D} \otimes \mathbf{F}_{2}$ itself. For two variables, the answer has been worked out by Walker and Xiao. We continue to use the notation $C$ generically for the quotient of the module by the hit elements, with the usual embellishments $C(n), C_{d}(n)$ when referring to the polynomial algebra in $n$ variables and the degree $d$.

EXAMPLE 7.8. For the action of $\mathscr{D} \otimes \mathbf{F}_{2}$ on $\mathbf{W}(2) \otimes \mathbf{F}_{2}$, a basis for $C(2)$ is given by the equivalence classes of the monomials $1, x_{1}, x_{2}, x_{1}^{2} x_{2}, x_{1}^{2^{n}-1} x_{2}$ for $n \geqslant 1$.

Unlike the rational case, the quotient $C$ in the modular case is no longer finite dimensional. It is interesting to contrast the situation for the differential operator algebra with the Steenrod algebra. Already, in the one-variable case, under the action of the Steenrod algebra, the elements $x^{2^{n}-1}$ are not hit, and form a vector space basis for $C(1)$. In the two-variable case, the original work of Peterson [158] gives the following result.

Example 7.9. For the action of $\mathscr{A}$ on $\mathbf{W}(2) \otimes \mathbf{F}_{2}$, a basis for $C(2)$ is given by the equivalence classes of the monomials

$$
x_{1}^{2^{k}-1} x_{2}^{2^{r}-1}
$$

for $k, r \geqslant 0$, and

$$
x_{1}^{2^{a}-1} x_{2}^{2^{a-1}-1+2^{a}\left(2^{b}-1\right)}
$$

for $a, b \geqslant 1$

In the general $n$-variable problem, there will be fewer elements in a basis of $C(n)$ for the action of $\mathscr{D} \otimes \mathbf{F}_{2}$ than for action of the Steenrod algebra. In particular, 'spikes', namely monomials of the form $x_{1}^{2^{\lambda_{1}}-1} x_{2}^{2^{\lambda_{2}}-1} \cdots x_{n}^{2^{\lambda_{n}}-1}$, are never hit under the action of the Steenrod algebra, and must be included in a basis of $C(n)$, but spikes can be hit under the action of the differential operator algebra. It is not clear what is the exact relationship between the hit problems for the two algebras $\mathscr{A}$ and $\mathscr{D} \otimes \mathbf{F}_{2}$ in the general $n$-variable case.

7.3 The hit problem for the Steenrod algebra. We shall confine our attention in this subsection to some specific results in the case of the hit problem for the Steenrod algebra. It can be seen directly that matrix substitution, in a given number of variables over the field of two elements, commutes with the action of the modulo 2 reduction of the differential operator

$$
D_{2^{k}-1}=\sum_{i \geqslant 1} x_{i}^{2^{k}} \frac{\partial}{\partial x_{i}},
$$

and also with wedge products of operators of this type. In other words, the action of the Steenrod algebra (on the left) of $\mathbf{W}(n) \otimes \mathbf{F}_{2}$ admits the full semigroup 
$M\left(n, \mathbf{F}_{2}\right)$ of all $n \times n$ matrices over $\mathbf{F}_{2}$ as symmetries acting on the right. This is the modular analogue of the semigroup $\Gamma_{n}$ of symmetries for the action of the differential operator algebra in the integral case. For the Steenrod algebra, the quotients $C_{d}(n)$ are representations of $M\left(n, \mathbf{F}_{2}\right)$ over the natural field $\mathbf{F}_{2}$ [217]. The equivariant version of the hit problem asks for a description of $C(n)$ as an $M\left(n, \mathbf{F}_{2}\right)$-module. Information in the three-variable case can be found in $[\mathbf{4 2}, \mathbf{5 0}, \mathbf{9 1}$, and progress on the general problem can be traced through the papers $[15,26,50,178,179,182,218,219]$. Problems still exist in four or more variables. The following results illustrate some of the progress.

Recall the numerical function $\mu(d)$ as in Definition 4.4, the least number $k$ for which it is possible to write $d=\sum_{1 \leqslant j \leqslant k}\left(2^{\lambda_{j}}-1\right)$, where $\lambda_{j} \geqslant 1$. Peterson [158] made the following conjecture, subsequently proved by the $\chi$-trick. hit.

THEOREM $7.10\left(\right.$ Wood [218]). If $\mu(d)>n$, then every element of $\mathbf{W}_{d}(n) \otimes \mathbf{F}_{2}$ is

Phrased another way, the theorem states that the dimension of $C_{d}(n)$ is zero unless $\mu(d) \leqslant n$. An odd prime analogue appears in [173].

A recent advance has been Judith Silverman's proof of the Singer conjecture.

THEOREM 7.11 (Silverman [178]). Let $u$ and $v$ be polynomials of degrees $e$ and $f$, respectively, and suppose $e<\left(2^{k+1}-1\right) \mu(f)$ for some $k \geqslant 0$. Then $u v^{k^{k+1}}$ is hit.

The proof uses the $\chi$-trick and Theorem 4.6.

In [42], an upper bound is proposed for the dimension of $C_{d}(n)$ for the prime 2 case in terms of a certain function of $n$. This has been generalised to the odd prime case by Crossley [51]. It remains a problem, however, to find the least upper bound. Kameko [91] conjectures in the prime 2 case that

$$
\operatorname{dim} C_{d}(n) \leqslant \prod_{i=1}^{n}\left(2^{i}-1\right),
$$

and Crossley conjectures in the odd prime case that the upper bound is

$$
\prod_{i=1}^{n}\left(2 p^{i-1}-1\right)
$$

In the prime 2 case, Kameko's conjecture is true for $n \leqslant 3$.

Among the most useful devices for handling the hit problem for the action of the Steenrod algebra on polynomials are the right and left lexicographic orderings of the weight vectors of monomials $x_{1}^{e_{1}} \cdots x_{n}^{e_{n}}$. Let $e=\sum_{i \geqslant 0} \alpha_{i}(e) 2^{i}$ denote the binary expansion of $e$, where $\alpha_{i}(e)$ is 0 or 1 . Then the weight vector of a monomial is defined by

$$
w\left(x_{1}^{e_{1}} \cdots x_{n}^{e_{n}}\right)=\left(w_{0}, w_{1}, \ldots, w_{k}, \ldots\right)
$$

where $w_{k}=\sum_{1 \leqslant i \leqslant n} \alpha_{k}\left(e_{i}\right)$. The weight vectors can then be ordered left or right lexicographically. This gives rise to two partial orderings of monomials. Both are especially well adapted to the right action of the full matrix semigroup and the left action of the Steenrod algebra. They fit in well with Young diagrams and representation theory of the symmetric groups and general linear groups. The 
following result is a sample application of these orderings that was made in [42]. Let $\alpha(e)=\sum_{i \geqslant 0} \alpha_{i}(e)$ denote the number of digits 1 in the binary expansion of $e$.

Theorem 7.12 (Carlisle and Wood [42]). For a fixed $n$, the $\mathscr{A}$-module $\mathbf{W}(n) \otimes \mathbf{F}_{2}$ is generated by monomials $x_{1}^{e_{1}} \cdots x_{n}^{e_{n}}$ where, up to permutation of the variables, $\alpha\left(e_{i}+1\right) \leqslant i$.

The set of generators exhibited by this result is not minimal, in general. Further information on the hit problem, using the homological approach, can be found in $[15,26,50,166]$.

7.4 Modular representations and splitting theory. From a topological point of view, $\mathbf{W}(n) \otimes \mathbf{F}_{2}$ is the cohomology of the product $X$ of $n$ copies of infinite real projective space, otherwise known as the classifying space $B(\mathbf{Z} / 2)^{n}$ of the group $(\mathbf{Z} / 2)^{n}$. The suspension of $X$ decomposes, up to homotopy type, into the one-point union of certain spaces $Y_{\rho}$, which are parametrised by the irreducible modular representations $\rho$ of the semigroup $M\left(n, \mathbf{F}_{2}\right)[39,79, \mathbf{1 3 5}, \mathbf{1 3 6}, \mathbf{2 1 6}, 217]$. The space $Y_{\rho}$ occurs $d(\rho)$ times in the one-point union, where $d(\rho)$ is the dimension of $\rho$. There are $2^{n}$ distinct irreducible representations of $M\left(n, \mathbf{F}_{2}\right)$ parametrised by the sequences of non-negative integers

$$
\lambda_{1} \geqslant \lambda_{2} \geqslant \ldots \geqslant \lambda_{n} \geqslant 0,
$$

subject to the constraints $\lambda_{i}-\lambda_{i+1} \leqslant 1$ for $1 \leqslant i<n$ and $\lambda_{n} \leqslant 1$. Such a sequence is called column 2-regular, and may be represented by a matrix in which position $(i, j)$ is occupied by a mark if $1 \leqslant j \leqslant \lambda_{i}$, and other positions are empty. This follows the standard convention for the Young diagram of a partition

$$
\lambda=\left(\lambda_{1}, \lambda_{2}, \ldots, \lambda_{n}\right)
$$

of the number $|\lambda|=\lambda_{1}+\lambda_{2}+\cdots+\lambda_{n}$, as used in the representation theory of symmetric groups [121].

The transposed matrix corresponds to the conjugate partition $\lambda^{\prime}$ of $|\lambda|$, where $\lambda_{i}^{\prime}$ is the number of rows $k$ such that $\lambda_{k} \geqslant i$. If $\lambda$ is column 2-regular, then $\lambda^{\prime}$ is strictly decreasing. In [40], a partition with strictly decreasing parts is referred to simply as 2-regular.

The first value of the degree $d$ for which a simple module $\rho$ occurs as a composition factor in $\mathbf{W}_{d}(n) \otimes \mathbf{F}_{2}$ gives the connectivity of the corresponding piece $Y_{\rho}$ in the splitting of the suspension of $X$. For the prime 2, the 'first occurrence problem' has been solved for all the simple modules.

Theorem 7.13 (Carlisle and Kuhn [40]). The simple $M\left(n, \mathbf{F}_{2}\right)$-module corresponding to the column 2-regular partition $\lambda$ occurs for the first time as a composition factor in $\mathbf{W}_{d}(n) \otimes \mathbf{F}_{2}$ when

$$
d=\sum_{j \geqslant 1} \lambda_{j}^{\prime} 2^{j-1},
$$

where $\lambda^{\prime}$ is the conjugate partition of $\lambda$.

There are analogues for splitting the stable type of $p$-localisations of the product 
of $n$ copies of infinite complex projective space, otherwise known as the classifying space $B T^{n}$ of the $n$-torus $[39,67,79,135,136,216,217]$.

Little is known about the odd prime analogue of the first occurrence problem, although a few cases are resolved $[40,41,62,216]$.

The first occurrence of a simple module as a submodule is also an interesting question, especially in conjunction with the problem of linking it to the first occurrence as composition factor via Steenrod operations. Again, for the prime 2, the first occurrence problem of an irreducible as a submodule has been solved by Schwartz $[\mathbf{1 7 0}]$ and Tri $[\mathbf{2 0 4}, \mathbf{2 0 3}]$. Information on the general problem in the context of Weyl modules can be found in [61].

THEOREM 7.14 (Schwartz [170]). The first occurrence of the simple $M\left(n, \mathbf{F}_{2}\right)$-module corresponding to the column 2-regular partition $\lambda$ as a submodule in $\mathbf{W}_{d}(n) \otimes \mathbf{F}_{2}$ is for

$$
d=\sum_{j \geqslant 1} \lambda_{j} 2^{j-1} .
$$

The first occurrence question is just one aspect of the general problem of describing the structure of the cohomology of the pieces $Y_{\rho}$ as modules over the Steenrod algebra. For each piece, there is a restricted hit problem. This modular restricted hit problem is difficult to solve even in the case of the trivial representation. Here, the submodule occurrences of the trivial representation in the polynomial algebra can be identified with the ring of invariants of the general linear group $\mathrm{GL}\left(n, \mathbf{F}_{2}\right)$. This is known classically as the Dickson algebra $[\mathbf{5 8}, \mathbf{1 2 3}, \mathbf{2 0 5}, \mathbf{2 1 4}]$, and is a polynomial subring of $\mathbf{W}(n) \otimes \mathbf{F}_{2}$ generated by the Dickson invariants.

The action of the Steenrod squares on the Dickson invariants and, more generally, invariants of parabolic subgroups of general linear groups, is worked out in a number of places $[9,81,83,99,146,193,214]$, and global product formulae are produced in [116]. The restricted hit problem for the Dickson algebra is solved for a small number of variables in $[\mathbf{7 2}, \mathbf{8 4}, \mathbf{8 7}, \mathbf{8 8}]$, but the general problem appears difficult. Hung conjectures that all elements in the positively graded Dickson algebra are hit in $\mathbf{W}(n)$ if the number of variables is at least three $[\mathbf{8 4}, \mathbf{8 3}, \mathbf{8 6}, \mathbf{8 5}]$.

The relationship between the Steenrod algebra $\mathscr{A}$ and the full matrix semigroups $M\left(n, \mathbf{F}_{2}\right)$ is similar to the relationship between the differential operator algebra $\mathscr{D}$ and the symmetric semigroups $\Gamma_{n}$. It would be interesting, therefore, to carry out a parallel development of splitting theory, at least at the algebraic level, with respect to the symmetric group and the differential operator algebra, posing hit problems and restricted hit problems in the rational and modular cases. Over the rationals, Specht's original paper [185] deals with the occurrences of $\Sigma_{n}$ modules in the polynomial algebra. James and Kerber [90] treat the theory of modular Specht modules and Weyl modules for the general linear groups. Information can be found in [69] for the relationship between first occurrence problems and Lannes' theory of unstable modules over the Steenrod algebra $[107,108,109,112,113]$.

We can ask for an analogue, for the differential operator algebra, of the AdamsGunawardena-Miller result [7] which states that all grade-preserving linear transformations of the polynomial algebra $\mathbf{W} \otimes \mathbf{F}_{2}$, which commute with the action of the Steenrod squares, are given by matrix substitution [217]. Finally, it is worth noting that the ring of invariants, over the integers, of a finite permutation group is 
a module over $\mathscr{D}$. It would be interesting to investigate this module structure in the case of modular rings of invariants of cyclic groups [75]. We refer to Larry Smith's book [183] for general background on invariant theory, and his recent survey article [184] for up to date information.

\subsection{Problems.}

Problem 7.15. Solve the hit problem for the differential operator algebra over the rationals. In other words, find a criterion on the polynomial $g$ for a solution of the differential equation

$$
S Q^{1} f_{1}+S Q^{2} f_{2}=g .
$$

Prove that the Artin basis is a basis of the cokernel $C$.

Problem 7.16. Give a description of how the differential operator algebra acts on the pieces of $\mathbf{W}(n) \otimes \mathbf{Q}$ split by a full set of idempotents of the symmetric semigroup $\Gamma_{n}$.

Problem 7.17. Solve the hit problem for the modulo $p$ reduction of the differential operator algebra, and investigate its relationship to modular representations of the symmetric group.

Problem 7.18. Investigate the relationship between the hit problems for the modulo 2 reduction of the differential operator algebra and the Steenrod algebra.

Problem 7.19. Prove Kameko's conjecture and the odd prime analogue.

Problem 7.20. Solve the first occurrence problems for the irreducible modules of general linear groups in the polynomial algebra at odd primes.

Problem 7.21. Solve the restricted hit problem for the Steenrod algebra acting on Dickson invariants.

Problem 7.22. Find an analogue of the Adams-Gunawardena-Miller result for the differential operator algebra in relation to the symmetric group.

Problem 7.23. Investigate the structure of modular rings of invariants of permutation groups as modules over $\mathscr{D}$.

\section{The dual of $\mathscr{A}(n)$ and graph theory}

In this section we show how various constructions in the dual of the finite Hopf algebra $\mathscr{A}(n)$ can be interpreted graphically. Milnor [131] defines $\mathscr{A}^{*}(n)$ as the quotient of the dual Steenrod algebra, generated additively by the equivalence classes of the monomials $\xi_{1}^{i_{1}} \xi_{2}^{i_{2}} \cdots \xi_{n+1}^{i_{n+1}}$, where $0 \leqslant i_{j}<2^{n+2-j}$ for $1 \leqslant j \leqslant n+1$. The multiplication is as usual for polynomials, except that a product is zero when any exponent exceeds the limited range; in other words, $\mathscr{A}^{*}(n)$ is a truncated polynomial 
algebra. It is a Hopf algebra under the coproduct and conjugation introduced in Section 1:

$$
\psi\left(\xi_{k}\right)=\sum_{0 \leqslant i \leqslant k} \xi_{k-i}^{2^{i}} \otimes \xi_{i}, \quad \chi\left(\xi_{k}\right)=\sum_{0 \leqslant i \leqslant k-1} \xi_{k-i}^{2^{i}} \chi\left(\xi_{i}\right) .
$$

Milnor solves the recursion formulae for conjugation to give

$$
\chi\left(\xi_{k}\right)=\sum_{\pi} \prod_{i=1}^{l(\pi)} \xi_{\pi(i)}^{2^{\sigma(i)}},
$$

where $\pi$ runs through ordered partitions of $k, \pi(i)$ denotes the $i$ th part of $\pi, l(\pi)$ is the length of $\pi$, and $\sigma(i)$ denotes the sum of the first $i-1$ parts of $\pi$ [131].

EXAMPLE 8.1. The ordered partitions of 3 are $(3),(2,1),(1,2),(1,1,1)$. Hence

$$
\chi\left(\xi_{3}\right)=\xi_{3}+\xi_{2} \xi_{1}^{4}+\xi_{1} \xi_{2}^{2}+\xi_{1} \xi_{1}^{2} \xi_{1}^{4} .
$$

We see, therefore, that $\mathscr{A}(n)^{*}$ is a connected finite graded Hopf algebra with unique non-zero element $\Delta_{n}=\xi_{1}^{2^{n+1}-1} \xi_{2}^{2^{n}-1} \cdots \xi_{n+1}$ in top grading $t_{n}=(n-1) 2^{n+2}+$ $n+5$. The total dimension of $\mathscr{A}^{*}(n)$ is $2^{(n+1)(n+2) / 2}$. A connected finite Hopf algebra is a Poincare algebra [125], which means, in particular, that for any element $\xi$ of $\mathscr{A}^{*}(n)$ in grading $r$, there is an element $\eta$ in grading $t_{n}-r$ such that $\xi \eta=\Delta_{n}$.

8.1 Graphical interpretation. We now explain a way of coding the truncated polynomial algebra $\mathscr{A}^{*}(n)$ in terms of graphs. To describe the coding, we fix an $n$ and consider the complete graph $\Delta$ on $n+1$ vertices which we label $1,2, \ldots, 2^{n}$. A subgraph in the present context will always include the full set of vertices, so that effectively a subgraph is a subset of the edges of $\Delta$. The edge joining $2^{i}$ to $2^{j}$ for $i<j$ is labelled with the element $\xi_{j-i}^{i}$ of $\mathscr{A}^{*}(n)$. The following figure illustrates $\mathscr{A}^{*}(2)$.

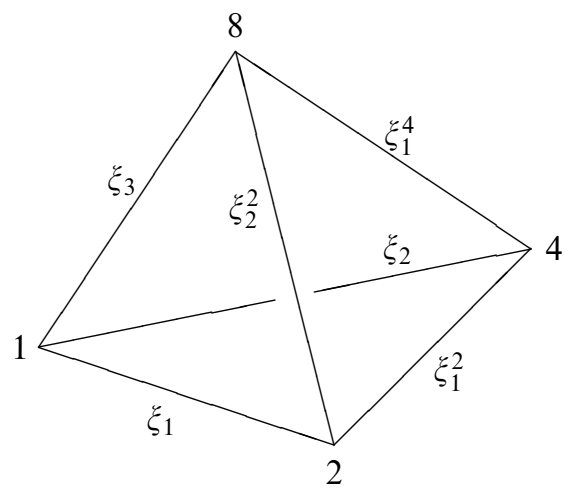

By writing the exponents of the $\xi_{j}$ in binary form, we see that the monomials $\xi_{1}^{i_{1}} \xi_{2}^{i_{2}} \cdots \xi_{n+1}^{i_{n+1}}$ of $\mathscr{A}^{*}(n)$ are in bijective correspondence with the subgraphs of $\Delta$. For example, in the above illustration, the set of edges labelled $\xi_{1}, \xi_{1}^{2}, \xi_{1}^{4}$ corresponds to the monomial $\xi_{1}^{7}$ (compare with the discussion of the stripping process in Subsection 5.1).

The following items illustrate a few elementary ways of interpreting graphical statements algebraically. 
- The monomial $\xi_{1}^{s_{1}} \xi_{2}^{s_{2}} \cdots \xi_{n}^{s_{n}}$ is a subgraph of $\xi_{1}^{r_{1}} \xi_{2}^{r_{2}} \cdots \xi_{n}^{r_{n}}$ if and only if $r_{i}$ dominates $s_{i}$ for each $i$; in other words, the binomial coefficient $\left(\begin{array}{l}r_{i} \\ s_{i}\end{array}\right)$ is odd (compare with Subsection 4.5). Hence the condition for a subgraph is that the product

$$
\prod_{i=1}^{i=n}\left(\begin{array}{l}
r_{i} \\
s_{i}
\end{array}\right)
$$

is odd.

- The product of two subgraphs in $\mathscr{A}^{*}(n)$ is the symmetric difference of the edge sets multiplied by the square of the intersection. In particular, the product of two disjoint graphs is their union.

- The conjugate of $\xi_{n}$ in $\mathscr{A}^{*}(n)$ is the sum of all circuit-free paths in $\Delta$ starting at vertex 1 and ending at vertex $2^{n}$.

- The coproduct of $\xi_{n}$ in $\mathscr{A}^{*}(n)$ is the sum of tensors of all pairs of edges which make length 2 paths from vertex 1 to vertex $2^{n}$ (counting $1 \otimes \xi_{n}$ and $\xi_{n} \otimes 1$ as degenerate length 2 paths).

- The identity of the algebra $\mathscr{A}^{*}(n)$ is the subgraph with no edges. The top element $\Delta_{n}$ of $\mathscr{A}^{*}(n)$ is identified with the complete graph $\Delta$ itself. The Poincare dual of a subgraph is the graph of complementary edges.

- The exponents of a monomial corresponding to a subgraph are obtained from the adjacency matrix of the graph by reading subdiagonals from bottom to top as binary expansions.

EXAMPLE 8.2. The adjacency matrix of the graph $\xi_{1}^{5} \xi_{2}^{2}$ in $\mathscr{A}^{*}(2)$ is

$$
\left(\begin{array}{llll}
0 & 1 & 0 & 0 \\
1 & 0 & 0 & 1 \\
0 & 0 & 0 & 1 \\
0 & 1 & 1 & 0
\end{array}\right) .
$$

The subdiagonals read $101_{2}=5,10_{2}=2$ and $0=0$, giving the exponents of the monomial as required.

A similar graph-theoretic interpretation can be made of the Hopf algebra $\mathscr{A}(n)$ itself by associating the element $P_{t}^{s}$ with the edge joining $2^{s}$ to $2^{s+t}$. Under the wedge product, $\mathscr{A}(n)$ is an exterior algebra. The product of two subgraphs this time is their union if the graphs are distinct, and zero if they intersect. The coproduct is the same as before, and the remarks on Poincare duality still apply.

The composition product of two graphs labelled by the elements $P_{t}^{s}$ is more difficult to interpret. The same applies if we use the elements $S q^{2^{5}\left(2^{t}-1\right)}$ in place of $P_{t}^{s}$ as the labels.

The action of the Steenrod algebra on polynomials also has a graph-theoretic interpretation. This time we label the vertices of $\Delta$ by integers $0,1, \ldots, n$, and the edge joining $i$ to $j$, for $i<j$, by $x_{j-i}^{2^{i}}$. From the Cartan formula in Theorem 1.1, it follows that the total Steenrod squaring operation

$$
S q=S q^{0}+S q^{1}+\cdots+S q^{n}+\cdots
$$

is given on a monomial by

$$
\frac{S q\left(x_{1}^{i_{1}} x_{2}^{i_{2}} \cdots x_{n}^{i_{n}}\right)}{x_{1}^{i_{1}} x_{2}^{i_{1}} \cdots x_{n}^{i_{n}}}=\left(1+x_{1}\right)^{i_{1}}\left(1+x_{2}\right)^{i_{2}} \cdots\left(1+x_{n}\right)^{i_{n}} .
$$


This expansion gives a list of all subgraphs of $x_{1}^{i_{1}} x_{2}^{i_{2}} \cdots x_{n}^{i_{n}}$.

\subsection{Problems.}

Problem 8.3. Compile a dictionary translating the language of graph theory into the language of the truncated polynomial algebra $\mathscr{A}^{*}(n)$.

Problem 8.4. How are trees identified in $\mathscr{A}^{*}(n)$ ?

Problem 8.5. What does it mean algebraically for a monomial $\xi_{1}^{i_{1}} \xi_{2}^{i_{2}} \cdots \xi_{n+1}^{i_{n+1}}$ in $\mathscr{A}^{*}(n)$ to be connected when viewed as a graph?

Problem 8.6. Is there an algebraic interpretation in $\mathscr{A}^{*}(n)$ of classical questions concerning Hamiltonian circuits in graphs?

Problem 8.7. How can the composition product in $\mathscr{A}(n)$ be interpreted graphically?

Problem 8.8. Can the dual of the differential operator algebra $\mathscr{D}$ be given a graphical interpretation, perhaps in terms of weighted graphs?

\section{The Steenrod group}

In this section we consider the group of units $U(\mathscr{A})$ in the Steenrod algebra, and refer to it as the Steenrod group. Since the maximal ideal of elements of positive grading in $\mathscr{A}$ is a nil-ring, an element of $U(\mathscr{A})$ has the form $1+x$, where $x$ has positive grading. In fact, $U(\mathscr{A})$ is a 2-group. The order of $1+x$ is the first power of 2 not less than the nilpotence height of $x$. Furthermore, $U(\mathscr{A})$ is locally finite because it is filtered by the finite groups $U(n)=U(\mathscr{A}(n))$, associated with the finite Hopf subalgebras $\mathscr{A}(n)$ of the Steenrod algebra. The conjugation, composed with inversion, is an automorphism of $U(\mathscr{A})$ compatible with the subgroups $U(n)$, and is known to be outer [168].

9.1 The group $U(1)$. Clearly, $U(0)$ is the cyclic group of order 2 generated by $1+S q^{1}$. The next group, $U(1)$, is more interesting. Recall the Z-basis of $\mathscr{A}(1)$ which was explained in Definition 4.4. By selecting substrings of the top element $S q^{2} S q^{3} S q^{1}$ of $\mathscr{A}(1)$, we produce the following list of seven elements in $U(1)$ :

$$
\begin{gathered}
a_{1}=1+S q^{1}, \quad a_{2}=1+S q^{2}, \quad a_{3}=1+S q^{3}, \quad a_{4}=1+S q^{2} S q^{1}, \\
a_{5}=1+S q^{3} S q^{1}, \quad a_{6}=1+S q^{2} S q^{3}, \quad a_{7}=1+S q^{2} S q^{3} S q^{1} .
\end{gathered}
$$

As a vector space, $\mathscr{A}(1)$ has dimension $2^{3}=8$. Hence the group $U(1)$ has order $2^{7}$. The collection of the $a_{i}$ forms a basis for $U(1)$ in the following sense.

LEMma 9.1. The elements of U(1) can be written uniquely in the form

$$
a_{1}^{\varepsilon_{1}} a_{2}^{\varepsilon_{2}} \cdots a_{7}^{\varepsilon_{7}},
$$

where each $\varepsilon_{i}$ is 0 or 1 . 
A set of defining relations for $U(1)$ is then worked out from the Adem relations. In the usual group theory notation for commutators,

$$
\left[a_{1}, a_{2}\right]=a_{3} a_{4} a_{5} a_{6} a_{7}, \quad\left[a_{1}, a_{3}\right]=\left[a_{1}, a_{4}\right]=a_{5}, \quad\left[a_{2}, a_{3}\right]=\left[a_{2}, a_{4}\right]=a_{6},
$$

and all other commutators $\left[a_{i}, a_{j}\right]$ not listed are 1 . In addition,

$$
a_{2}^{2}=a_{5}, \quad a_{3}^{2}=a_{4}^{2}=a_{7},
$$

and other squares $a_{i}^{2}$ not listed are 1. In principle, these relations allow the product of two elements in $U(1)$ to be expressed in the form of Lemma 9.1. Information about the group $U(1)$ has been obtained by Bob Sandling in Manchester and Don Coleman in Kentucky using the MAGMA package [38]. It is a 3-generated group of nilpotence class 2 and exponent 4 with $\left\{a_{1}, a_{2}, a_{3}\right\}$ as a minimal set of generators. Its centre coincides with its Frattini subgroup, which is elementary abelian (of order 16), while its derived group is of order 8. It appears as group number 128.172 in Eamonn O'Brien's list [151]. The properties of $U(1)$ are not too difficult to verify by hand. However, the size of $U(n)$ grows rapidly with $n$. The vector space dimension of $\mathscr{A}(n)$ is $k=2^{(n+1)(n+2) / 2}$, and the order of $U(n)$ is therefore $2^{k-1}$.

9.2 Remarks. There are many subalgebras and Hopf subalgebras of the Steenrod algebra, each with its associated group of units. In principle, any module over the Steenrod algebra may be viewed as a module over the Steenrod group and its subgroups. This includes the cohomology of topological spaces and the rings of modular invariants of general linear groups. It would be interesting to see how far group-theoretic methods can be applied to elucidate some of the problems about the Steenrod algebra. For example, can results about nilpotence be deduced from results about the orders of elements in the Steenrod group? Topologists are interested in splitting classifying spaces of finite groups, as we discussed in Subsection 7.4. For general information on this subject, we refer to $[\mathbf{2 4}, \mathbf{1 2 6}, \mathbf{1 6 0}]$. Some recent work on splitting 2-groups can be found in [45]. The following problems include a somewhat self-referential item.

\subsection{Problems.}

Problem 9.2. How does the cohomology of the Steenrod group $U(1)$ split as a module over the Steenrod algebra?

Problem 9.3. Are there any special group-theoretic features which distinguish those groups arising from subalgebras and Hopf subalgebras of the Steenrod algebra? In particular, can the groups $U(n)$ be described group-theoretically?

Problem 9.4. Describe the Steenrod groups arising in the odd prime case.

Problem 9.5. Investigate the groups of units in modular reductions of the divided differential operator algebra $\mathscr{D}$.

\section{Computing in the Steenrod algebra}

Attempts at proving nilpotence conjectures for Steenrod squares by expansion in terms of the admissible basis or the Milnor basis are frustrated by the complexity 
of keeping track of binomial coefficients modulo 2 (see Subsection 6.1). Computer programs dedicated to this problem tend to consume a lot of time and memory in creating large lists which suddenly collapse at the last stage of the process. Nevertheless, the computer is invaluable as an interactive device for checking relations in the Steenrod algebra, and has long been a standard tool in the hands of experts [196] for the calculation of the homology of the Steenrod algebra. It is useful to have fast and efficient algorithms for expressing elements in the Steenrod algebra in a prescribed basis and for finding conjugates. Ken Monks [141] has a suite of programs in Maple for performing these tasks. However, the overheads in using an all-purpose package tend to reduce the scope of the applications, and it is sometimes necessary to resort to dedicated software in the $\mathrm{C}$ language for specific problems, especially over the field of two elements, for which the C language is particularly well adapted.

10.1 Computing conjugates. A nice exercise is the problem of reducing $\chi\left(S q^{k}\right)$ to admissible form. One method is to start with the smallest number $2^{r}-1$ not less than $k$ for which we know the answer is

$$
\chi\left(S q^{2^{r}-1}\right)=S q^{2^{r-1}} S q^{2^{r-2}} \cdots S q^{2} S q^{1},
$$

and then to strip iteratively by $\xi_{1}$ until we get down to $\chi\left(S q^{k}\right)$. This procedure never needs to do any rewriting, using the Adem relations, because admissibility is preserved at each stage. The program scans an admissible monomial, and for each adjacent pair of factors $S q^{i} S q^{j}$, it replaces $i$ by $i-1$ unless $i=2 j$, in which case the term is eliminated because $S q^{2 j-1} S q^{j}=0$. The only problem is the elimination of duplicates, and this is a common feature of all programming in the Steenrod algebra. Here is a dry run starting at $\chi\left(S q^{15}\right)$ and going down to $\chi\left(S q^{8}\right)$, stripping by $\xi_{1}$ at each stage.

$$
\begin{aligned}
& \text { ExAmPLE } 10.1 \\
& \qquad \begin{aligned}
\chi\left(S q^{15}\right) & =S q^{8} S q^{4} S q^{2} S q^{1} \\
\chi\left(S q^{14}\right) & =S q^{8} S q^{4} S q^{2}, \\
\chi\left(S q^{13}\right) & =S q^{8} S q^{4} S q^{1} \\
\chi\left(S q^{12}\right) & =S q^{8} S q^{3} S q^{1}+S q^{8} S q^{4} \\
\chi\left(S q^{11}\right) & =S q^{7} S q^{3} S q^{1}+S q^{8} S q^{2} S q^{1}+S q^{8} S q^{3}+S q^{8} S q^{3} \\
& =S q^{7} S q^{3} S q^{1}+S q^{8} S q^{2} S q^{1} \\
\chi\left(S q^{10}\right) & =S q^{6} S q^{3} S q^{1}+S q^{7} S q^{2} S q^{1}+S q^{7} S q^{3}+S q^{7} S q^{2} S q^{1}+S q^{8} S q^{2} \\
& =S q^{6} S q^{3} S q^{1}+S q^{7} S q^{3}+S q^{8} S q^{2}, \\
\chi\left(S q^{9}\right) & =S q^{6} S q^{2} S q^{1}+S q^{6} S q^{3}+S q^{6} S q^{3}+S q^{7} S q^{2}+S q^{7} S q^{2}+S q^{8} S q^{1} \\
& =S q^{6} S q^{2} S q^{1}+S q^{8} S q^{1} \\
\chi\left(S q^{8}\right) & =S q^{5} S q^{2} S q^{1}+S q^{6} S q^{2}+S q^{7} S q^{1}+S q^{8} .
\end{aligned}
\end{aligned}
$$

Any stage of the process can, of course, be stored and used as the starting point for another run. This illustrates once again the fundamental role played by the basic relations $S q^{2 j-1} S q^{j}=0$ and the stripping technique. The above program is efficient for numbers not far down from $2^{k}-1$, and is tolerable up to about $S q^{64}$, but becomes very slow for higher powers of 2 and numbers immediately above. For 
such numbers, Straffin's formula in Theorem 4.17 and related formulae discussed in Subsection 4.5 are preferable but require further processing to bring to admissible form.

10.2 Nilpotence questions. The general problem of reducing an element $E$ of the Steenrod algebra to admissible form is a standard exercise in list processing. It can be viewed in terms of a large matrix $M$ of non-negative integers. The rows of $M$ are the items of the list, and entries of a row of $M$ are the exponents of a monomial in Steenrod squares. The list may therefore be identified with an element in the Steenrod algebra. The number of columns in $M$ is taken as the longest length of a monomial in the initial list, and may be pre-assigned because reduction to admissible form via the Adem relations does not increase the length of monomials. The number of rows in $M$ will depend on the size of the job. At each stage of the process, an Adem program scans the rows of $M$ in adjacent column pairs, and applies an Adem relation if the pair is not admissible, thereby expanding each item of the list into a new list. The old item is discarded, the new list is concatenated, and duplicates are eliminated. The process stops when all rows of the matrix are admissible. Questions of efficiency then arise as to the best method of scanning the matrix, as well as more technical problems to do with the $\mathrm{C}$ language. For example, it is debatable whether one should commandeer a large static array for the whole process and arrange one's own garbage collection using standard linked list techniques, or whether one should use the built-in pointer system, structures of the $\mathrm{C}$ language and dynamic allocation.

Checking the relation $\left(S q^{32}\right)^{12}=0$ is tedious, and the relation $\left(S q^{128}\right)^{16}=0$ would seem to be beyond the capacity of the PC, although Ken Monks achieved this on a large machine after days of central processing time. As far as these particular nilpotence results are concerned, we know from the ideal Conjecture 6.10 that the situation can be eased slightly by checking $\left(S q^{2^{n}}\right)^{2 n} S q^{1}=0$, which requires less processing. However, it is not difficult to pose a fairly modest looking problem about nilpotence which will create more items in the list during processing than there are particles in the universe. Of course, we now know the right way to handle $\left(S q^{2^{n}}\right)^{2 n+2}=0$. Here is the shortest dry run to show that $\left(S q^{8}\right)^{8}=0$.

EXAMPLE 10.2. Stripping by the vector (8) and post-multiplying by $S q^{8}$ shows that it is sufficient to demonstrate $\widehat{S q^{8}}\left(S q^{8}\right)^{7}=0$. Now $\widehat{S q^{8}} S q^{8}=S q^{12} \widehat{S q^{4}}$ (see Example 4.15). Hence it is sufficient to prove $\widehat{S q^{4}}\left(S q^{8}\right)^{6}=0$. Repeat the routine to replace the problem by the equivalent statement $\widehat{S q^{4} S q^{8}}\left(S q^{8}\right)^{5}=\widehat{S q^{4}} S q^{12} \widehat{S q^{4}}\left(S q^{8}\right)^{4}=0$, and use the relation $\widehat{S q^{4}} S q^{12}=S q^{14} \widehat{S q^{2}}$ to reduce the problem to $\widehat{S q^{2}} \widehat{S q^{4}}\left(S q^{8}\right)^{4}=0$. Repeat the routine to replace the problem by the equivalent statement

$$
\widehat{S q^{2}} \widehat{S q^{4}} \widehat{S q^{8}}\left(S q^{8}\right)^{3}=\widehat{S q^{2}} \widehat{S q^{4}} S q^{12} \widehat{S q^{4}}\left(S q^{8}\right)^{2}=\widehat{S q^{2}} S q^{14} \widehat{S q^{2}} \widehat{S q^{4}}\left(S q^{8}\right)^{2}=0,
$$

and use the relation $\widehat{S q^{2}} S q^{14}=S q^{15} \widehat{S q^{1}}$ to reduce the problem to $\widehat{S q^{1}} \widehat{S q^{2}} \widehat{S q^{4}}\left(S q^{8}\right)^{2}$ $=0$. Repeat one final time to prove the result.

Each stage of the argument is kept as tight as possible, and there is no increase in the size of lists. However, lacking the special devices exhibited in the above example, the problem of exponential growth in list size remains. For example, how far can the statement $\left(S q^{2^{n}-2}\right)^{n+1}=0$, for $n>2$, be verified on the computer? Some of the 
problems listed below have come out of computer sessions, but faith in the results depends more on the uniformity of the pattern than on the computer evidence, which is rather scanty.

\subsection{Problems.}

Problem 10.3. Prove or disprove:

$$
\begin{aligned}
& \left(S q^{2^{n}}\right)^{2 n} S q^{1}=0 \\
& \left(S q^{2^{n}}\right)^{2 n+1}=\left(S q^{2^{n+1}+2^{n-1}} S q^{2^{n+1}+2^{n-2}} \cdots S q^{2^{n+1}+2} S q^{2^{n+1}+1}\right) S q^{1}, \\
& \left(S q^{2^{n}}\right)^{2 n+1}=S q^{3 \cdot 2^{n-1}} S q^{7 \cdot 2^{n-2}} \cdots S q^{\left(2^{n}-1\right) \cdot 2} S q^{\left(2^{n+1}-1\right) \cdot 1} S q^{\left(2^{n+1}-1\right)}, \\
& \left(S q^{2^{n}}\right)^{2 n+1}=\left(S q^{2^{n}}\right)^{2 n-1} S q^{2^{n+1}-1} S q^{1}, \\
& \left(S q^{2^{n}}\right)^{2 n+1}=\chi\left(S q^{2^{n-1}}\right)\left(S q^{2^{n+1}}\right)^{n} S q^{1} .
\end{aligned}
$$

Problem 10.4. Is it true that every monomial in the admissible expansion of $\left(S q^{2^{n}}\right)^{2 n}$ has length precisely $n+1$ ?

Problem 10.5. Prove or disprove: for $n>2$,

$$
\begin{aligned}
& \left(S q^{2^{n}-2}\right)^{n+1}=0, \\
& \left(S q^{2^{n}-2}\right)^{n} S q^{2^{n-1}-1}=0, \\
& \left(S q^{2^{n}-2}\right)^{n} S q^{2^{n-1}-2} S q^{2^{n-1}}=0 .
\end{aligned}
$$

Problem 10.6. Is there a sensible formula for the height of an atomic square, or indeed an arbitrary square?

Problem 10.7. Find an efficient method of computing conjugates of monomials in Steenrod squares and products of elements with conjugates of elements.

Problem 10.8. Develop programs for handling relations among integral squares.

\section{References}

1. E. ABE, Hopf algebras (Cambridge University Press, 1980).

2. J. F. Adams, 'On the structure and applications of the Steenrod algebra', Comment. Math. Helv. 32 (1958) $180-214$.

3. J. F. Adams, 'On the non-existence of elements of Hopf invariant one', Ann. of Math. 72 (1960) 20-104.

4. J. F. Adams, 'On formulae of Thom and Wu', Proc. London Math. Soc. 11 (1961) 741-752.

5. J. F. Adams, Stable homotopy and generalised homology, Chicago Lectures in Math. (University of Chicago Press, 1974).

6. J. F. ADAMS, 'Operations of the $n$th kind in $K$-theory and what we don't know about $R P^{\infty}$ ', New developments in topology, London Math. Soc. Lecture Note Ser. 11 (Cambridge University Press, 1974) $1-9$.

7. J. F. Adams, J. Gunawardena and H. Miller, 'The Segal conjecture for elementary abelian 2-groups', Topology 24 (1985) 435-460.

8. J. F. Adams and H. R. Margolis, 'Sub-Hopf algebras of the Steenrod algebra', Proc. Cambridge Philos. Soc. 76 (1974) 45-52

9. J. F. AdAms and C. Wilkerson, 'Finite H-spaces and algebras over the Steenrod algebra', Ann. of Math. 111 (1980) 95-143.

10. J. AdEm, 'The iteration of Steenrod squares in algebraic topology', Proc. Nat. Acad. Sci. U.S.A. 38 (1952) $720-726$. 
11. J. ADEM, 'The relations in Steenrod powers of cohomology classes', Algebraic geometry and topology, Symposium in honour of S. Lefschetz (Princeton University Press, 1957).

12. A. Adem and R. J. Milgram, Cohomology of finite groups, Grundlehren Math. Wiss. 309 (Springer, New York, 1994).

13. M. Aigner, Combinatorial theory, Grundlehren Math. Wiss. 234 (Springer, New York, 1979).

14. T. AIKaWA, 'The products in the Steenrod rings of the complex and symplectic cobordism theories', Publ. Res. Inst. Math. Sci. Kyoto 10 (1974/5) 581-600.

15. M. A. Alghamdi, M. C. Crabb and J. R. Hubbuck, 'Representations of the homology of $B V$ and the Steenrod algebra I', Adams Memorial Symposium on algebraic topology, Vol. 2, London Math. Soc. Lecture Note Ser. 176 (Cambridge University Press, 1992) 217-234.

16. D. J. Anick and F. P. Peterson, ' $A$ *annihilated elements in $H_{*}\left(\Omega \Sigma\left(R P^{\infty}\right)\right)^{\prime}$, Proc. Amer. Math Soc. 117 (1993) 243-250.

17. D. Arnon, 'Monomial bases in the Steenrod algebra', J. Pure Appl. Algebra 96 (1994) 215-223.

18. E. Artin, Galois theory, Notre Dame Math. Lectures 2 (University of Notre Dame Press, Notre Dame, IN, 1944).

19. M. F. Atiyah and F. Hirzebruch, 'Cohomologie-Operationen und charakteristische Klassen' Math. Z. 77 (1961) 149-187.

20. M. G. Barratt and H. Miller, On the anti-automorphism of the Steenrod algebra, Contemp. Math 12 (Amer. Math. Soc., Providence, RI, 1981) 47-52

21. D. BAusum, 'An expression for $\chi\left(S q^{m}\right)$ ', Preprint, Minnesota University (1975).

22. A. F. BEARDON, Iteration of rational functions, Grad. Texts in Math. 132 (Springer, New York, 1991).

23. D. J. Benson, Representations and cohomology II: Cohomology of groups and modules, Cambridge Stud. Adv. Math. 31 (Cambridge University Press, 1991).

24. D. J. Benson, 'Stably splitting BG', Bull. Amer. Math. Soc. 33 (1996) 189-198.

25. T. Bisson and A. Joyal, 'The Dyer-Lashof algebra in bordism', C. R. Math. Rep. Acad. Sci. Canada 17 (1995) 135-140.

26. J. M. Boardman, 'Modular representations on the homology of powers of real projective spaces', Algebraic Topology, Oaxtepec 1991, Contemp. Math. 146 (Amer. Math. Soc., Providence, RI, 1993) 49-70.

27. A. Borel, Algebraic $\mathscr{D}$-modules (Academic Press, London, 1987).

28. A. Borel and J.-P. Serre, 'Groupes de Lie et puissances réduites de Steenrod', Amer. J. Math. 75 (1953) 409-448.

29. R. BotT, 'Symmetric products and Steenrod squares', Ann. of Math. 57 (1953) 579-590.

30. E. Brown, D. Davis and F. Peterson, 'The homology of $B O$ and some results about the Steenrod algebra', Math. Proc. Cambridge Philos. Soc. 81 (1977) 393-398.

31. E. Brown and F. Peterson, 'Some remarks about symmetric functions', Proc. Amer. Math. Soc. 60 (1976) 349-352.

32. M. BüGER, 'On the composition of polynomials of the form $z^{2}+c_{n}$ ', Math. Ann., to appear.

33. V. M. Buhstaber, Semigroups of maps into groups, operator doubles, and complex bordism, Adv. Soviet Math. 22 (Amer. Math. Soc., Providence, RI, 1995)

34. V. M. Buhstaber and A. N. Kholodov, 'Groups of formal diffeomorphisms of the superline, generating functions for sequences of polynomials and functional equations', Math. USSR Izv 35 (1990) 277-305.

35. V. M. Buhstaber and A. V. Shokurov, 'The Landweber-Novikov algebra and formal vector fields on the line', transl. from Funktsional. Anal. i Prilozhen. 12 (3) (1978) 1-11.

36. S. R. Bullett and I. G. Macdonald, 'On the Adem relations', Topology 21 (1982) 329-332.

37. P. J. Cameron, Combinatorics (Cambridge University Press, 1996).

38. J. Cannon and C. Playoust, 'An introduction to MAGMA', University of Sydney (1993).

39. D. P. Carlisle, P. Eccles, S. Hilditch, N. Ray, L. Schwartz, G. Walker and R. Wood, 'Modular representations of GL( $n, p)$, splitting $\Sigma\left(C P^{\infty} \times \cdots \times C P^{\infty}\right)$ and the $\beta$-family as framed hypersurfaces', Math. Z. 189 (1985) 239-261.

40. D. P. Carlisle and N. J. KuHN, 'Subalgebras of the Steenrod algebra and the action of matrices on truncated polynomial algebras', J. Algebra 121 (1989) 370-387.

41. D. P. CARLISLE and G. WALKER, 'Poincare series for the occurrence of certain modular representations of $\operatorname{GL}(n, p)$ in the symmetric algebra', Proc. Roy. Soc. Edinburgh Sect. A 113 (1989) $27-41$.

42. D. P. CARLISLE and R. M. W. Wood, 'The boundedness conjecture for the action of the Steenrod algebra on polynomials', Adams Memorial Symposium on algebraic topology, Vol. 2, London Math. Soc. Lecture Note Ser. 176 (Cambridge University Press, 1992) 203-216.

43. H. Cartan, 'Une théorie axiomatique des carrés de Steenrod', C. R. Acad. Sci. Paris $230(1950)$ 425-427.

44. H. Cartan, 'Sur l'itération des opérations de Steenrod', Comment. Math. Helv. 29 (1955) 40-58.

45. M. Catalano, 'Some stable splittings of $B P$ for some $P$ of order a power of 2', J. Pure Appl. Algebra 111 (1996) 31-50 
46. P. E. Cohen, 'On the Adem relations', Proc. Cambridge Philos. Soc. 57 (1961) 265-267.

47. L. Comtet, Advanced combinatorics (D. Reidel, Dordrecht, 1974).

48. S. C. Coutinho, A primer of algebraic D-modules, London Math. Soc. Stud. Texts 33 (Cambridge University Press, 1995).

49. M. C. Crabb, M. D. Crossley and J. R. Hubbuck, ' $K$-theory and the anti-automorphism of the Steenrod algebra', Proc. Amer. Math. Soc. 124 (1996) 2275-2281.

50. M. C. Crabb and J. R. Hubbuck, 'Representations of the homology of $B V$ and the Steenrod algebra II', Algebraic topology: new trends in localization and periodicity (San Feliu de Guixols 1994), Progr. Math. 136 (Birkhäuser, Boston, 1996) 143-154.

51. M. D. Crossley, ' $H * V$ is of bounded type over $\mathscr{A}_{p}$ ', Preprint, Barcelona (1996).

52. M. D. Crossley, ' $\mathscr{A}(p)$-annihilated elements of $H_{*}\left(C P^{\infty} \times C P^{\infty}\right)$ ', Math. Proc. Cambridge Philos. Soc. 120 (1996) 441-453.

53. M. D. Crossley and J. Hubbuck, 'Not the Adem relations', Bol. Soc. Mat. Mexicana 37 (1992) 99-107.

54. M. D. Crossley and S. Whitehouse, 'On conjugation invariants in the dual Steenrod algebra', Preprint, Paris XIII (1998).

55. D. M. DAvis, 'The antiautomorphism of the Steenrod algebra', Proc. Amer. Math. Soc. 44 (1974) 235-236.

56. D. M. DAVIS, 'Some quotients of the Steenrod algebra', Proc. Amer. Math. Soc. 83 (1981) 616-618.

57. D. M. DAvis, 'On the height of $S q^{2^{n}}$, , Preprint, Lehigh University, Bethlehem, PA (1985).

58. L. E. Dickson, 'A fundamental system of invariants of the general modular linear group with a solution of the form problem', Trans. Amer. Math. Soc. 12 (1911) 75-98.

59. J. Dieudonné, A history of algebraic and differential topology 1900-1960 (Birkhäuser, Basel, 1989).

60. A. Dold, 'Über die Steenrodschen Kohomologieoperationen', Ann. of Math. 73 (1961) 258-294.

61. S. Doty and G. WALKer, 'The composition factors of $F_{p}\left[x_{1}, x_{2}, x_{3}\right]$ as a GL(3, $\left.p\right)$-module', J. Algebra 147 (1992) 411-441.

62. S. Doty and G. WALker, 'Truncated symmetric powers and modular representations of $\mathrm{GL}_{n}$, Math. Proc. Cambridge Philos. Soc. 119 (1996) 231-242.

63. J. Duflot, 'Lots of Hopf algebras', Preprint (1996).

64. P. J. ECCles, 'Multiple points of codimension one immersions of oriented manifolds', Math. Proc. Cambridge Philos. Soc. 87 (1980) 213-220.

65. P. J. ECCles, 'Codimension one immersions and the Kervaire invariant one problem', Math. Proc. Cambridge Philos. Soc. 90 (1981) 483-493.

66. P. J. ECCLES, 'Characteristic numbers of immersions and self-intersection manifolds', Topology with applications, Szekszárd (Hungary), Bolyai Soc. Math. Stud. 4 (János Bolyai Math. Soc., Budapest, 1993) 197-216.

67. P. J. Eccles and W. P. R. Mitchell, 'Splitting $\Sigma\left(C P^{\infty} \times C P^{\infty}\right)$ localised at 2', Quart. J. Math. Oxford 39 (1988) 285-289.

68. P. J. Eccles and W. P. R. Mitchell, 'Triple points of immersed orientable $2 n$-manifolds in 3n-space', J. London Math. Soc. 39 (1989) 335-346.

69. V. Franjou and L. Schwartz, 'Reduced unstable $A$-modules and the modular representation theory of the symmetric groups', Ann. Sci. École Norm. Sup. 23 (1990) 593-624.

70. A. M. Gallant, 'Excess and conjugation in the Steenrod algebra', Proc. Amer. Math. Soc. 76 (1979) 161-166.

71. L. Geissinger, Hopf algebras of symmetric functions and class functions, Lecture Notes in Math. 579 (Springer, New York, 1977) 168-181.

72. V. Giambalvo, N. H. V. Hung and F. P. Peterson, ' $H^{*}\left(R P^{\infty} \times \cdots \times R P^{\infty}\right)$ as a module over the Steenrod algebra', Hilton Symposium 1993, Montreal, CRM Proc. Lecture Notes 6 (Amer. Math. Soc., Providence, RI, 1994) 133-140.

73. V. Giambalvo and F. P. Peterson, On the height of $S q^{2^{n}}$, Contemp. Math. 181 (Amer. Math. Soc., Providence, RI, 1995) 183-186.

74. V. Giambalvo and F. P. Peterson, 'The annihilator ideal of the action of the Steenrod algebra on $H^{*}\left(R P^{\infty}\right)$ ', Topology Appl. 65 (1995) 105-122.

75. M. GöBEL, 'Computing bases for rings of permutation invariant polynomials', J. Symbolic Comput 19 (1995) 285-291.

76. K. R. GoOdEARL and R. B. WARFIELD, An introduction to noncommutative noetherian rings, London Math. Soc. Stud. Texts 16 (Cambridge University Press, 1989).

77. I. P. Goulden and D. M. JACKson, Combinatorial enumeration (Wiley, New York, 1983).

78. B. Gray, Homotopy theory (Academic Press, London, 1975).

79. J. C. HARRIS and N. J. KuHN, 'Stable decomposition of classifying spaces of finite abelian $p$ groups', Math. Proc. Cambridge Philos. Soc. 103 (1988) 427-449.

80. P. Henrici, Applied and computational analysis, Vol. 1 (Wiley, New York, 1988).

81. T. J. HewetT, 'Modular invariant theory of parabolic subgroups of $\mathrm{GL}_{n}\left(\mathbf{F}_{q}\right)$ and the associated Steenrod modules', Duke Math. J. 82 (1996) 91-102. 
82. W-C. Hsiang, 'On Wu's formula of Steenrod squares on Stiefel-Whitney classes', Bol. Soc. Mat. Mexicana 8 (1963) 20-25.

83. N. H. V. Hung, 'The action of Steenrod squares on the modular invariants of linear groups', Proc. Amer. Math. Soc. 113 (1991) 1097-1104.

84. N. H. V. Hung, 'The action of the mod $p$ Steenrod operations on the modular invariants of linear groups', Vietnam J. Math. 23 (1995) 39-56.

85. N. H. V. HuNG, 'On the weak conjecture on spherical classes', Preprint (1996).

86. N. H. V. Hung, 'Spherical classes and the algebraic transfer', Trans. Amer. Math. Soc., to appear.

87. N. H. V. Hung and F. P. Peterson, 'A-generators for the Dickson algebra', Trans. Amer. Math Soc. 347 (1995) 4687-4728.

88. N. H. V. Hung and F. P. Peterson, 'Spherical classes and the Dickson algebra', Math. Proc Cambridge Philos. Soc., to appear.

89. N. JACOBSON, Lie algebras, Interscience Tracts Pure Appl. Math. (Wiley, New York, 1962).

90. D. J. JAMES and A. KerBer, The representation theory of the symmetric group, Encyclopaedia Math. 16 (Addison-Wesley, Reading, MA, 1981).

91. M. Kameko, 'Products of projective spaces as Steenrod modules', Thesis, Johns Hopkins University (1990).

92. R. Kane, 'Brown-Peterson operations and Steenrod modules', Quart. J. Math. Oxford 30 (1979) 455-467.

93. M. Kaneda, M. Shimada, M. Tezuka and N. Yagita, 'Representations of the Steenrod algebra' J. Algebra 155 (1993) 435-454.

94. I. KaraCA, 'The nilpotent height of $P_{t}^{s}$ for odd primes', Preprint, Lehigh University, Bethlehem, PA (1996).

95. M. Karoubi, 'Formes différentiales non-commutatives et opérations de Steenrod', Topology 34 (1995) 699-715.

96. M. Karoubi, 'Formes différentiales non-commutatives et cohomologie à coefficients arbitraires', Trans. Amer. Math. Soc. 347 (1995) 4277-4299.

97. C. Kassel, Quantum groups, Grad. Texts in Math. (Springer, New York, 1995).

98. J. Katsura, Y. Shimiza and K. Ueno, 'Complex bordism ring and conformal field theory over $Z$ ', Math. Ann. 291 (1991) 551-571.

99. N. Kechagias, 'The Steenrod algebra action on generators of subgroups of $\mathrm{GL}_{n}(Z / p Z)$ ', Proc. Amer. Math. Soc. 118 (1993) 943-952.

100. J. Klippenstein and L. Lomonaco, 'On the coefficients of the double total squaring operation', Bol. Soc. Mat. Mexicana 37 (1992) 309-316.

101. S. O. Kochman, Bordism, stable homotopy and the Adams spectral sequences, Fields Inst. Monogr. 7 (Amer. Math. Soc., Providence, RI, 1996).

102. I. Kozma, 'Irreducibles in the Landweber-Novikov algebra', Proc. Amer. Math. Soc. 55 (1976) 453-456.

103. D. Kraines, 'On excess in the Milnor basis', Bull. London Math. Soc. 3 (1971) 363-365.

104. L. Kristensen, 'On secondary cohomology operations', Colloq. on algebraic topology (Publ. Math. Inst., Aarhus Univ., 1962) 16-21.

105. L. Kristensen, 'On the cohomology of two-stage Postnikov systems', Acta Math. 107 (1962) $73-123$.

106. L. KRISTENSEN, 'On a Cartan formula for secondary cohomology operations', Math. Scand. 16 (1965) 97-115.

107. N. J. KUHN, 'Generic representations of the finite general linear groups and the Steenrod algebra', Amer. J. Math. 116 (1994) 327-360.

108. N. J. KuHn, 'Generic representations of the finite general linear groups and the Steenrod algebra: II', K-theory 8 (1994) 395-428.

109. N. J. KuHN, 'Generic representations of the finite general linear groups and the Steenrod algebra: III', K-theory 9 (1995) 273-303.

110. T. LANCE, 'Steenrod and Dyer-Lashof operations on BU', Trans. Amer. Math. Soc. 129 (1967) 94-110.

111. P. S. Landweber, 'Cobordism operations and Hopf algebras', Trans. Amer. Math. Soc. 276 (1983) 497-510.

112. J. Lannes and L. Schwartz, 'Sur la structure des $\mathscr{A}$-modules instable injectifs', Topology 28 (1989) $153-169$.

113. J. Lannes and S. Zarati, 'Sur les $\mathscr{U}$-injectifs', Ann. Sci. École Norm. Sup. 19 (1986) 593-603.

114. C. Lenart, 'The combinatorics of Steenrod operations on the cohomology of Grassmannians', Preprint, MIT, Cambridge, MA (1997).

115. Z. LI, 'Formulas for Brown-Peterson operations', Canad. J. Math. 46 (1994) 772-792.

116. Z. LI, 'Product formulas for Steenrod operations', Proc. Edinburgh Math. Soc. 38 (1995) 207-232.

117. J. K. Lin, 'Generalisation of Peterson's and other formulas', Adv. in Math. Beijing 14 (1985) $348-355$. 
118. I. Llerena and H. V. Hung, 'The complete Steenrod algebra and the generalised Dickson algebra', Algebraic topology: new trends in localization and periodicity (San Feliu de Guixols 1994), Progr. Math. 136 (Birkhäuser, Boston, 1996) 271-284.

119. L. Lomonaco, 'A basis of admissible monomials for the universal Steenrod algebra', Ricerche Mat. 40 (1991) 137-147.

120. L. Lomonaco, 'The iterated total squaring operation', Proc. Amer. Math. Soc. 115 (1992) 1149-1155.

121. I. G. Macdonald, Symmetric functions and Hall polynomials, Oxford Math. Monographs (2nd edn, Clarendon Press, Oxford, 1995).

122. P. A. MACMAhon, Combinatorial analysis (reprint, Chelsea, New York, 1960).

123. I. MadSen and J. R. Milgram, The classifying spaces for surgery and cobordism of manifolds, Ann. of Math. Stud. 92 (Princeton University Press, 1979).

124. S. MAJID, Foundations of quantum group theory (Cambridge University Press, 1995).

125. H. Margolis, Spectra and the Steenrod algebra, North-Holland Math. Library 29 (Elsevier, Amsterdam, 1983)

126. J. Martino, 'Classifying spaces and their maps', Homotopy theory and its applications (Cocoyoc 1993), Contemp. Math. 188 (Amer. Math. Soc., Providence, RI, 1995) 161-198.

127. H. Matsumura, Commutative algebra (Benjamin/Cummings, Reading, MA, 1980).

128. J. P. MAY, 'A general algebraic approach to Steenrod operations', The Steenrod algebra and its applications, Lecture Notes in Math. 168 (Springer, New York, 1970) 153-231.

129. J. C. MCConNell and J. C. Robson, Noncommutative noetherian rings (Wiley, Chichester, 1987).

130. C. M. McCrory, Geometric homology operations, Stud. Algebraic Topology, Adv. Math., Suppl. 5 (1978) $120-140$.

131. J. MiLnOR, 'The Steenrod algebra and its dual', Ann. of Math. 67 (1958) 150-171.

132. J. Milnor, 'On the bordism ring $\Omega^{*}$ and a complex analogue', Amer. J. Math. 82 (1960) 505-521.

133. J. Milnor and J. C. MoORE, 'On the structure of Hopf algebras', Ann. of Math. 81 (1965) 211-264.

134. J. Milnor and J. D. Stasheff, Characteristic classes, Ann. of Math. Stud. 76 (Princeton University Press, 1974).

135. S. A. Mitchell, 'Finite complexes with $A(n)$ free cohomology', Topology 24 (1985) 227-248.

136. S. A. Mitchell, 'Splitting $B(Z / p)^{n}$ and $B T^{n}$ via modular representation theory', Math. Z. 189 (1985) 285-298

137. K. Mizuno and Y. Saito, 'Note on the relations on Steenrod squares', Proc. Japan Acad. 35 (1959) $557-564$.

138. K. G. MonKs, 'Status report on the height of $S q^{2^{n}}$, Preprint, University of Scranton, PA (1991)

139. K. G. Monks, 'Nilpotence in the Steenrod algebra', Bol. Soc. Mat. Mexicana 37 (1992) 401-416.

140. K. G. Monks, 'Polynomial modules over the Steenrod algebra and conjugation in the Milnor basis', Proc. Amer. Math. Soc. 122 (1994) 625-634.

141. K. G. Monks, 'STEENROD: a Maple package for computing with the Steenrod algebra', monks@uofs.edu. (1995).

142. K. G. Monks, 'The nilpotence height of $P_{t}^{s}$, Proc. Amer. Math. Soc. 124 (1996) 1296-1303.

143. K. G. Monks, 'Change of basis, monomial relations, and the $P_{t}^{s}$ bases for the Steenrod algebra', J. Pure Appl. Algebra 125 (1998) 235-260.

144. S. Montgomery, Hopf algebras and their actions on rings, CBMS Regional Conf. Ser. in Math. 82 (Amer. Math. Soc., Providence, RI, 1993).

145. R. E. Mosher and M. C. TANGORA, Cohomology operations and applications in homotopy theory (Harper and Row, New York, 1968).

146. H. MUI, 'Dickson invariants and Milnor basis of the Steenrod algebra', Topology, theory and application, Colloq. Math. Soc. János Bolyai 41 (North-Holland, Amsterdam, 1985) 345-355.

147. S. Mukohdi, 'On a theorem of Toda in the Steenrod algebra', Mem. Fac. Sci. Kyushu Univ. Ser. A 14 (1960) 85-97.

148. A. Negishi, 'On exact sequences in the Steenrod algebra', J. Math. Soc. Japan 10 (1958) 71-78.

149. S. P. Novikov, 'The methods of algebraic topology from the point of view of cobordism theory', Izv. Akad. Nauk SSSR Ser. Mat. 31 (1967) 855-951; transl. by A. Liulevicius and N. Stein, Aarhus Lecture Notes (1968).

150. S. P. Novikov, 'Different doubles of the Hopf algebras, operator algebras on the quantum groups, complex bordism', Uspekhi Akad. Nauk SSSR 47 (1992) 189-190.

151. E. O'BRIEN, 'The groups of order 256', J. Algebra 143 (1991) 219-235.

152. S. PAPASTAVRIDIS, 'A formula for the obstruction to transversality', Topology 11 (1972) 415-416.

153. S. PapastavRIDIS, Relations in the mod p Steenrod algebra, Stud. Algebraic Topology, Adv. Math. Suppl. 5 (1978) 169-188.

154. H.-O. Peitgen and P. H. Richter, The beauty of fractals (Springer, Berlin, 1986).

155. D. J. Pengelley and F. Williams, 'Sheared algebra maps and operation Hopf algebras for modulo 2 homology and cohomology', Preprint, New Mexico State University, Las Cruces, NM (1997).

156. F. P. Peterson, 'Some formulas in the Steenrod algebra', Proc. Amer. Math. Soc. 45 (1974) 291-294.

157. F. P. Peterson, 'A modulo $p$ Wu formula', Bol. Soc. Mat. Mexicana 20 (1975) 56-58. 
158. F. P. Peterson, 'Generators of $\mathbf{H}^{*}\left(R P^{\infty} \wedge R P^{\infty}\right)$ as a module over the Steenrod algebra', Abstracts Amer. Math. Soc. (1987) 833-55-89.

159. F. P. Peterson, 'A -generators for certain polynomial algebras', Math. Proc. Cambridge Philos. Soc. 105 (1989) 311-312.

160. S. PrIDDY, 'On characterising summands in the classifying space of a group', Amer. J. Math. 112 (1990) 737-748.

161. D. QuilLEN, 'Elementary proofs of some results of cobordism theory using Steenrod squares', Adv. Math. 7 (1971) 29-56.

162. D. C. Ravenel, Complex cobordism and stable homotopy groups of spheres (Academic Press, London, 1986).

163. N. RAY, 'A topological calculus for formal power series', Preprint, University of Manchester (1997)

164. N. RAY and W. R. SchmitT, 'Combinatorial models for coalgebraic structures', Preprint, University of Manchester (1997).

165. N. RaY and W. R. Schmitt, 'Combinatorial models for Steenrod comodules', Preprint, University of Manchester (1997).

166. J. RepKa and P. Selick, 'On the subalgebra of $H_{*}\left(\left(\mathbb{R} P^{\infty}\right)^{n} ; \mathbb{F}_{2}\right)$ annihilated by Steenrod operations', J. Pure Appl. Algebra 127 (1998) 273-288.

167. M. Richardson and P. A. Smith, 'Periodic transformations of complexes', Ann. of Math. 39 (1938) 611-633.

168. R. SANDLING, 'The unit group of the Steenrod algebra', Preprint, University of Manchester (1997).

169. W. R. Sснмiтt, 'Incidence Hopf algebras', J. Pure Appl. Algebra 96 (1994) 299-330.

170. L. Schwartz, Unstable modules over the Steenrod algebra and Sullivan's fixed point set conjecture, Chicago Lectures in Math. (Chicago University Press, 1994).

171. J.-P. Serre, 'Cohomologie modulo 2 des complexes d'Eilenberg-MacLane', Comment. Math. Helv. $27(1953) 198-231$

172. B. ShaY, 'Modulo $p \mathrm{Wu}$ formulas for the Steenrod algebra and the Dyer-Lashof algebra', Proc. Amer. Math. Soc. 63 (1977) 339-347.

173. C. Shengmin and S. Xinyao, 'On the action of Steenrod powers on polynomial algebras', Proc Barcelona Conf. Algebraic Topology, Lecture Notes in Math. 1509 (Springer, New York, 1991) 326-330.

174. J. Silverman, 'Conjugation and excess in the Steenrod algebra', Proc. Amer. Math. Soc. 119 (1993) $657-661$.

175. J. Silverman, 'Hit polynomials and the canonical antiautomorphism', Proc. Amer. Math. Soc. 123 (1995) 627-637.

176. J. Silverman, 'Multiplication and combinatorics in the Steenrod algebra', Preprint, University of Michigan (1994)

177. J. Silverman, 'Stripping and conjugation in the Steenrod algebra', J. Pure Appl. Algebra 121 (1997) 95-106.

178. J. Silverman, 'Hit polynomials and conjugation in the dual Steenrod algebra', Proc. Math. Cambridge Philos. Soc. 123 (1998) 531-547.

179. J. Silverman and W. Singer, 'On the action of Steenrod squares on polynomial algebras II', J. Pure Appl. Algebra 98 (1995) 95-103.

180. W. Singer, 'Invariant theory and the lambda algebra', Trans. Amer. Math. Soc. 280 (1983) 673-693.

181. W. Singer, 'The transfer in homological algebra', Math. Z. 202 (1989) 493-523.

182. W. Singer, 'On the action of Steenrod squares on polynomial algebras', Proc. Amer. Math. Soc. 111 (1991) 577-583.

183. L. Sмith, Polynomial invariants of finite groups, Res. Notes Math. 6 (A. K. Peters, Wellesley, MA, 1995).

184. L. Smith, 'Polynomial invariants of finite groups, a survey of recent developments', Bull. Amer. Math. Soc. 34 (1997) 211-250.

185. W. SPecht, 'Die Irreduziblen Darstellungen der symmetrischen Gruppe', Math. Z. 42 (1935) 696711.

186. N. E. Steenrod, 'Products of cocycles and extensions of mappings', Ann. of Math. 48 (1947) 290-320.

187. N. E. SteEnrod, 'Reduced powers of cohomology classes', Ann. of Math. 56 (1952) 47-67.

188. N. E. SteEnRoD, 'Homology groups of symmetric groups and reduced power operations', Proc. Nat. Acad. Sci. U.S.A. 39 (1953) 213-217.

189. N. E. Steenrod and D. B. A. Epstein, Cohomology operations, Ann. of Math. Stud. 50 (Princeton University Press, 1962).

190. R. E. Stong, Notes on cobordism theory (Princeton University Press, 1968).

191. P. D. Straffin, 'Identities for conjugation in the Steenrod algebra', Proc. Amer. Math. Soc. 49 (1975) $253-255$.

192. T. Sugawara, 'Wu formulas for the modulo 3 reduced power operations', Mem. Fac. Sci. Kyushu Univ. 33 (1979) 297-309.

193. N. Sum, 'Steenrod operations on the modular invariants', Kodai Math. J. 17 (1994) 585-595. 
194. M. E. SweEdLer, Hopf algebras (Benjamin, New York, 1969).

195. R. M. Switzer, Algebraic topology, homotopy and homology, Grundlehren Math. Wiss. 212 (Springer, New York, 1975).

196. M. C. Tangora, 'Computing the homology of the lambda algebra', Mem. Amer. Math. Soc. 337 (1985).

197. R. Thом, 'Classes caracteristique et $i$-carrés', C. R. Acad. Sci. Paris 230 (1950) 427-429.

198. R. Thом, 'Une théorie intrinsèque des puissances de Steenrod', Colloq. Topologie Strasbourg (Publ. Math. Inst. Univ. Strasbourg, 1951)

199. R. Tном, 'Éspaces fibrés en sphères et carrés de Steenrod', Ann. Sci. École Norm. Sup. 69 (1952) $109-182$.

200. R. Tном, 'Quelque propriétés globales des variétés différentiables', Comment. Math. Helv. 28 (1954) $17-86$.

201. H. TodA, 'On exact sequences in the Steenrod algebra', Mem. Coll. Sci. Univ. Kyoto Ser. A Math 31 (1958) 33-64.

202. T. Tom DiEck, 'Steenrod Operationen in Koborbordismen-Theorie', Math. Z. 107 (1968) 380-401.

203. T. T. TRI, 'The irreducible modular representations of semigroups of all matrices', Acta Math. Vietnam. 20 (1995) 43-53.

204. T. T. TRI, 'On a conjecture for the first occurrence of irreducible modular representations of general linear groups', Preprint (1996).

205. P. TuRnER, 'Dickson coinvariants and the homology of $Q S^{0}$, Math. Z. 224 (1997) 209-228.

206. G. WAlker, 'Modular Schur functions', Trans. Amer. Math. Soc. 346 (1994) 569-603.

207. G. WALKER, 'The elements $\chi\left(P^{a}\right) P^{b}$ and $P^{a} \chi\left(P^{b}\right)$ ', Preprint, University of Manchester (1997).

208. G. WALKER, 'Some product and antipode formulae for the divided differential operator algebra', Preprint, University of Manchester (1997).

209. G. Walker and R. M. W. Wood, 'The nilpotence height of Sq $q^{2^{n}}$, Proc. Amer. Math. Soc. 124 (1996) 1291-1295.

210. G. WALKER and R. M. W. WoOD, 'The intersection of the admissible basis and the Milnor basis of the Steenrod algebra', J. Pure Appl. Algebra, to appear.

211. G. WALKer and R. M. W. Wood, 'The rational hit problem for differential operators and the Steenrod algebra', Preprint, University of Manchester (1997).

212. G. Walker and R. M. W. Wood, 'The nilpotence height of $P^{p^{n}}$, Math. Proc. Cambridge Philos. Soc. 123 (1998) 85-93.

213. C. T. C. WALL, 'Generators and relations for the Steenrod algebra', Ann. of Math. 72 (1960) 429-444.

214. C. Wilkerson, A primer on the Dickson invariants, Contemp. Math. 19 (Amer. Math. Soc., Providence, RI, 1983) 421-434.

215. C. Wilkerson, 'Classifying spaces, Steenrod operations and algebraic closure', Topology 16 (1997) $227-237$.

216. R. M. W. Wood, 'Modular representations of $\operatorname{GL}\left(n, \mathbf{F}_{p}\right)$ and homotopy theory', Algebraic Topology Göttingen 1984, Lecture Notes in Math. 1172 (Springer, New York, 1985) 188-203.

217. R. M. W. Wood, 'Splitting $\Sigma\left(C P^{\infty} \times \cdots \times C P^{\infty}\right)$ and the action of Steenrod squares on the polynomial ring $\mathbf{F}_{2}\left[x_{1}, \ldots, x_{n}\right]$ ', Algebraic Topology Barcelona 1986, Lecture Notes in Math. 1298 (Springer, New York, 1987) 237-255.

218. R. M. W. Wood, 'Steenrod squares of polynomials and the Peterson conjecture', Math. Proc. Cambridge Philos. Soc. 105 (1989) 307-309.

219. R. M. W. Wood, 'Steenrod squares of polynomials', Advances in homotopy theory, London Math Soc. Lecture Note Ser. 139 (Cambridge University Press, 1989) 173-177.

220. R. M. W. Wood, 'A note on bases and relations in the Steenrod algebra', Bull. London Math. Soc. 27 (1995) 380-386.

221. R. M. W. Wood, 'Differential operators and the Steenrod algebra', Proc. London Math. Soc. 75 (1997) 194-220.

222. W-T. Wu, 'Classes caracteristiques et carrés de Steenrod', C. R. Acad. Sci. Paris 230 (1950) 508-511.

223. W-T. Wu, 'Les $i$-carrés dans une variété grassmannienne', C. R. Acad. Sci. Paris 230 (1950) 918-920.

224. W-T. Wu, 'Sur les puissances de Steenrod', Colloq. Topologie Strasbourg (Publ. Math. Inst. Univ. Strasbourg, 1952).

225. W-T. Wu, 'On the relations between Smith operations and Steenrod powers', Fund. Math. 44 (1957) 263-269.

226. G-T. Yo, 'The Smith algebra', Acta Math. Sinica 7 (1957) 235-241.

Department of Mathematics

University of Manchester

Manchester M13 9PL 